\title{
MULTI-CIRCLE DETECTIONS FOR AN AUTOMATIC MEDICAL DIAGNOSIS SYSTEM
}

\author{
A Thesis \\ presented to \\ the Faculty of California Polytechnic State University, \\ San Luis Obispo
}

\author{
In Partial Fulfillment \\ of the Requirements for the Degree \\ Master of Science in Electrical Engineering
}

by

Dingran Lu

June 2012 
(C) 2012

Dingran Lu

ALL RIGHTS RESERVED 


\section{COMMITTEE MEMBERSHIP}

TITLE:

AUTHOR:

DATE SUBMITTED:

COMMITTEE CHAIR:

COMMITTEE MEMBER:

COMMITTEE MEMBER: Xiaomin Jin, Ph.D.

John A. Saghri, Ph.D.
Multi-circle Detections for an Automatic Medical Diagnosis System

Dingran Lu

June 2012 


\section{ABSTRACT \\ Multi-circle Detections for an Automatic Medical Diagnosis System \\ Dingran Lu}

Real-time multi-circle detection has been a challenging problem in the field of biomedical image processing, due to the variable sizes and non-ideal shapes of cells in microscopic images. In this study, two new multi-circle detection algorithms are developed to facilitate an automatic bladder cancer diagnosis system: one is a modified circular Hough Transform algorithm integrated with edge gradient information; and the other one is a stochastic search approach based on real valued artificial immune systems. Computer simulation results show both algorithms outperform traditional methods such as the Hough Transform and the geometric feature based method, in terms of both precision and speed.

Keywords: Circle detection, Hough Transform, Artificial Immune System, bladder cancer 


\section{ACKNOWLEDGMENTS}

First and foremost, I want to thank my advisor Professor Xiao-Hua (Helen) Yu for the introduction and guidance to this exciting Computational Intelligence field.

I would like to thank Professor John Saghri and Professor Xiaomin Jin for advice to this study and serving on my committee as well.

I also want to thank Mr. Bin Li from Smartbead Inc. for this unique opportunity and generous offering of the microscope image dataset.

I appreciate all kinds of help from my friends during my graduate study. Most importantly, I want to dedicate this study to my parents, who have always been supporting me. 


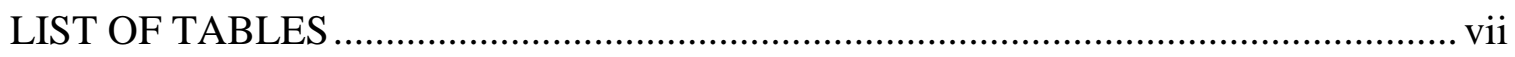

LIST OF FIGURES ………............................................................................... viii

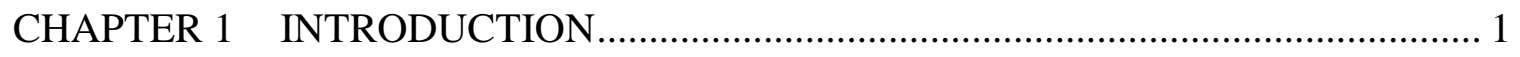

CHAPTER 2 LITERATURE REVIEW ................................................................... 4

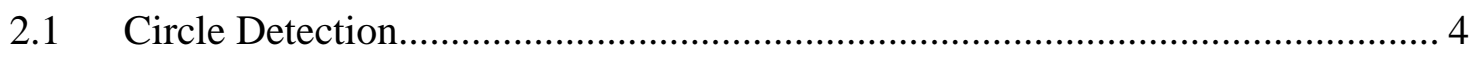

2.1.1 Geometric Feature Based Circle Detection...................................................... 5

2.1.2 Hough Transform Based Circle Detection...................................................... 7

2.1.3 Computational Intelligence Based Circle Detection...................................... 13

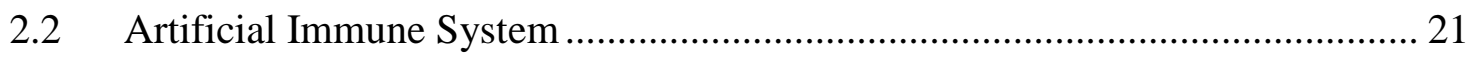

2.2.1 Natural Immune System Principles .............................................................. 21

2.2.2 Development of Artificial Immune System................................................ 24

CHAPTER 3 ALGORITHM DEVELOPMENT ………………………………....... 29

3.1 Original Prototype Algorithm ......................................................................... 29

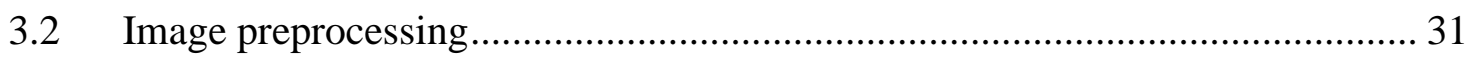

3.3 Geometric property based algorithm.............................................................. 35

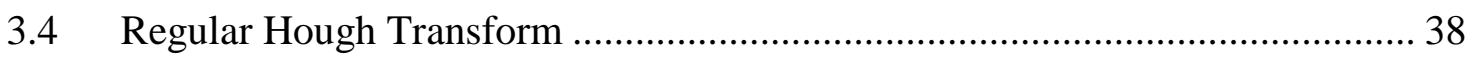

3.5 Modified Hough Transform with gradient information ...................................... 42

3.6 Real valued Artificial Immune System ............................................................... 46

CHAPTER 4 SIMULATION RESULTS............................................................... 58

CHAPTER 5 CONCLUSION AND FUTURE WORK................................................. 75

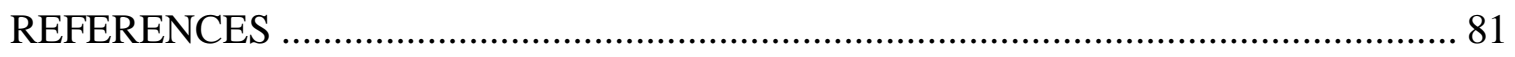

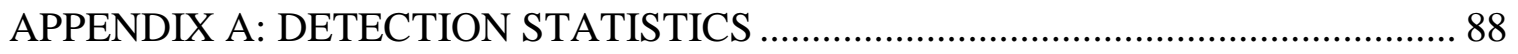

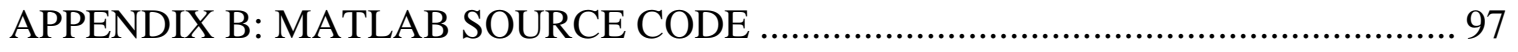




\section{LIST OF TABLES}

Table

Table 2-1 Applications of Artificial Immune System................................................. 27

Table 3-1 Comparison between binary and real valued AIS ........................................ 55

Table 3-2 Comparison between Genetic Algorithm and Artificial Immune System........ 57

Table 4-1 Confusion matrix of precision and sensitivity ........................................... 58

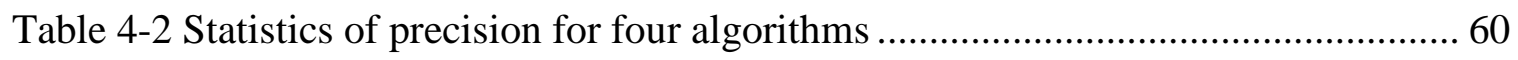

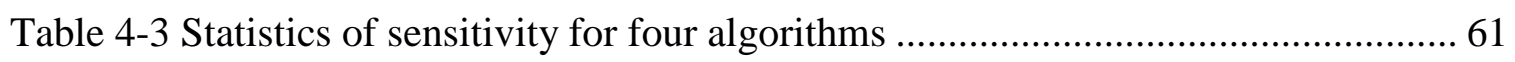

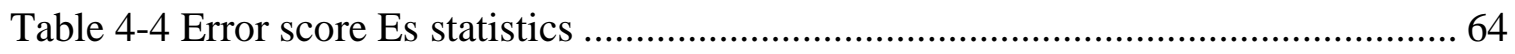

Table 5-1 Comparison of four circle detection algorithms ........................................ 78

Table 5-2 Grading rule for single bead's cell binding condition .................................. 80

Table A-1 Manual grading of detection for geometric feature based method ................. 88

Table A-2 Manual grading of detection for regular Hough Transform .......................... 90

Table A-3 Manual grading of detection for Modified Hough Transform ...................... 92

Table A-4 Manual grading of detection for Artificial Immune System ......................... 94 


\section{LIST OF FIGURES}

Figure Page

Figure 1.1 Microscopic image of bladder cancer cells binding with smart beads............. 2

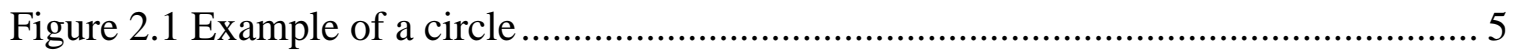

Figure 2.2 Schematic of semi-random search method [25] ....................................... 6

Figure 2.3 Example of straight line Hough Transform [31] ....................................... 8

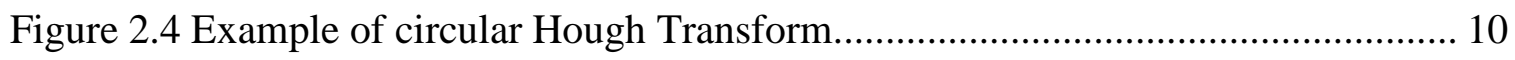

Figure 2.5 Functional components of human immune system [61] .............................. 22

Figure 2.6 Cells of natural immune system [61]..................................................... 23

Figure 2.7 Negative Selection principles [65] ...................................................... 25

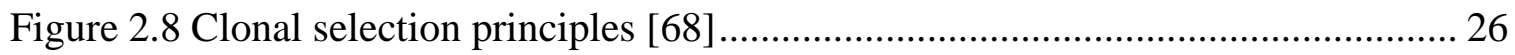

Figure 3.1 Successful example of first prototype algorithm ...................................... 30

Figure 3.2 Failed example of first prototype algorithm ............................................ 31

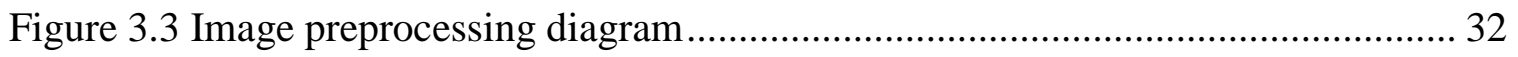

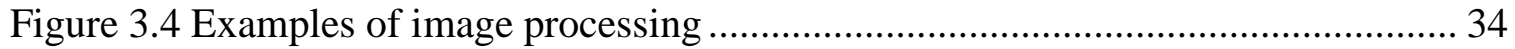

Figure 3.5 Function block diagram of geometric based circle detection algorithm ......... 36

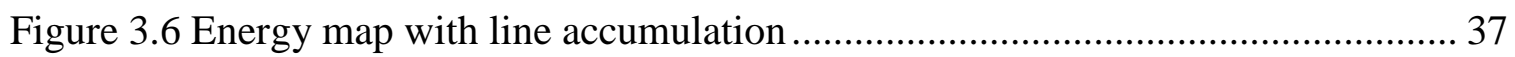

Figure 3.7 Final detected beads and radius histogram examples................................. 37

Figure 3.8 Function block diagram of regular Hough Transform realization.................. 39

Figure 3.9 Filtered reference layer of Hough Transform parameter space ...................... 40

Figure 3.10 Peak searching and final detection of regular Hough Transform.................. 41

Figure 3.11 Accumulation with radius and gradient orientation in a parameter layer ..... 43

Figure 3.12 Function block diagram of Modified Hough Transform ............................. 44

Figure 3.13 Reference layer of Modified Hough Transform parameter space ................ 45

Figure 3.14 Peak searching and final detection of modified Hough Transform.............. 46

Figure 3.15 Neighborhood of 8 -connectivity rule ...................................................... 47

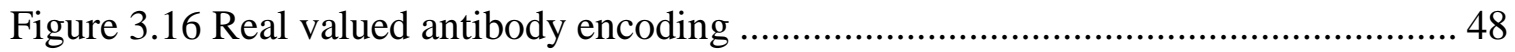




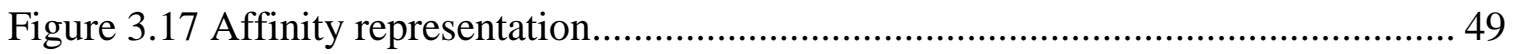

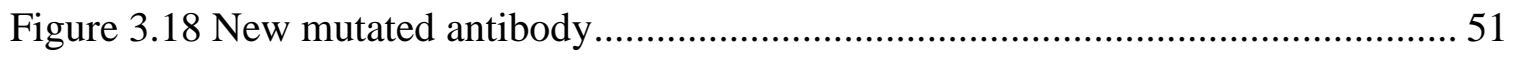

Figure 3.19 Control mechanism of $\Delta$ for real valued antibody ................................. 52

Figure 3.20 Function block diagram of Clonal Selection Algorithm ............................ 53

Figure 3.21 Edge map before and after Clonal Selection Algorithm ............................ 54

Figure 3.22 Circle Detection results with real valued Clonal Selection Algorithm ......... 54

Figure 3.23 Comparison of GA (left) and AIS (right) ............................................. 57

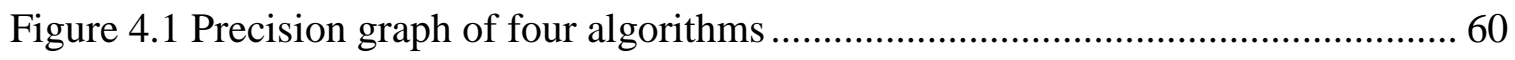

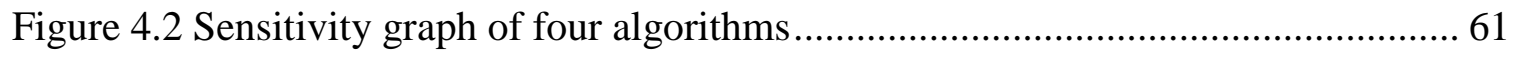

Figure 4.3 Precision and sensitivity graph of four algorithms ..................................... 62

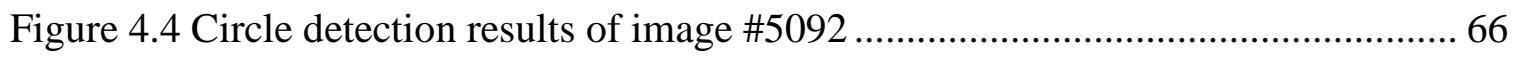

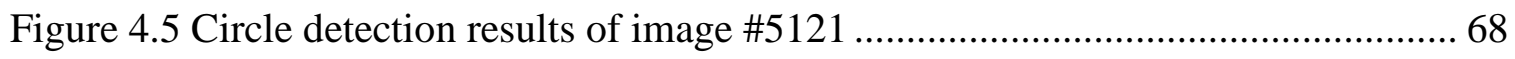

Figure 4.6 Circle detection results of image \#5133 ................................................... 70

Figure 4.7 Circle detection results of image \#5169 ................................................... 72

Figure 4.8 Example of traffic sign detection using four algorithms ............................. 74

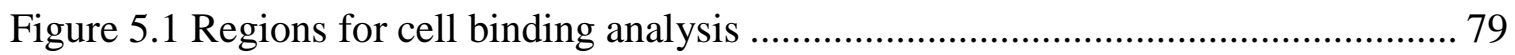




\section{CHAPTER 1 INTRODUCTION}

Bladder cancer is the malignant tumor from the urothelium of the urinary bladder. It is the fourth most common cancer in men and ninth in women [11]. According to American Cancer Society, an estimated 73,510 new cases of bladder cancer are expected to occur in 2012, and an estimated 14,880 deaths will occur in the same year [1].

Currently bladder cancer is commonly diagnosed by microscopic examination of cell samples from urine or bladder tissue, or examination of the bladder wall with a cystoscope inserted through the urethra [1]. With the development of medical device technology and computer aided diagnosis, bladder cancer cells can be checked automatically via many different ways, for example, CT scan [2], fluorescence cell label [3][4], cell mass spectra [5], cell impedance [6], and microscopic images [7][8][9].

Smartbead Inc. possesses a technology called PLZ4 (amino acid sequence: cQDGRMGFc) which is a kind of peptide that can selectively bind to bladder cancer cells but not to normal urothelial cells [10][11]. Thanks to this invention, the occurrence of bladder cancer can be detected by checking bladder cells attached to those PLZ4 coated smart beads from urine samples under a microscope, as shown in Figure 1.1. The biding

condition of cancer cells on smart beads is divided into 5 grades to represent different illness levels for diagnosis purpose.

This technology makes the detection of bladder cancer totally non-invasive and simpler comparing to other ways. Initially this bladder cancer diagnosis technique requires a technician to analyze these smart beads’ binding condition; more specifically, to manually verify how much surface area of a bead is covered by cancer cells, which 
makes it a slow process especially when there are dozens of beads and thousands of cells within view.

To automate this manual grading analysis, a computer program is developed to process the digital microscopic images. Since the smart beads' locations indicated where the cancer cells are, the main challenge is to find the smart beads, and then set them as a region of interest (ROI) for further analysis of cell binding conditions. Once those beads are accurately located and confirmed as the ROI, some image segmentation methods can be implemented to segment cells according to ROI, and binding grade can be approximated by the ratio of cell area over bead area.

The exact locations of the smart beads are very important, because only the cells attached to bead's surface are considered as cancer cells, and cells on the background are normal cells. For example, only cells on the circular bead's circumference and within the circumference are used for grading. Therefore, the first step of acquiring ROI can be simplified to a multi-circle detection problem.

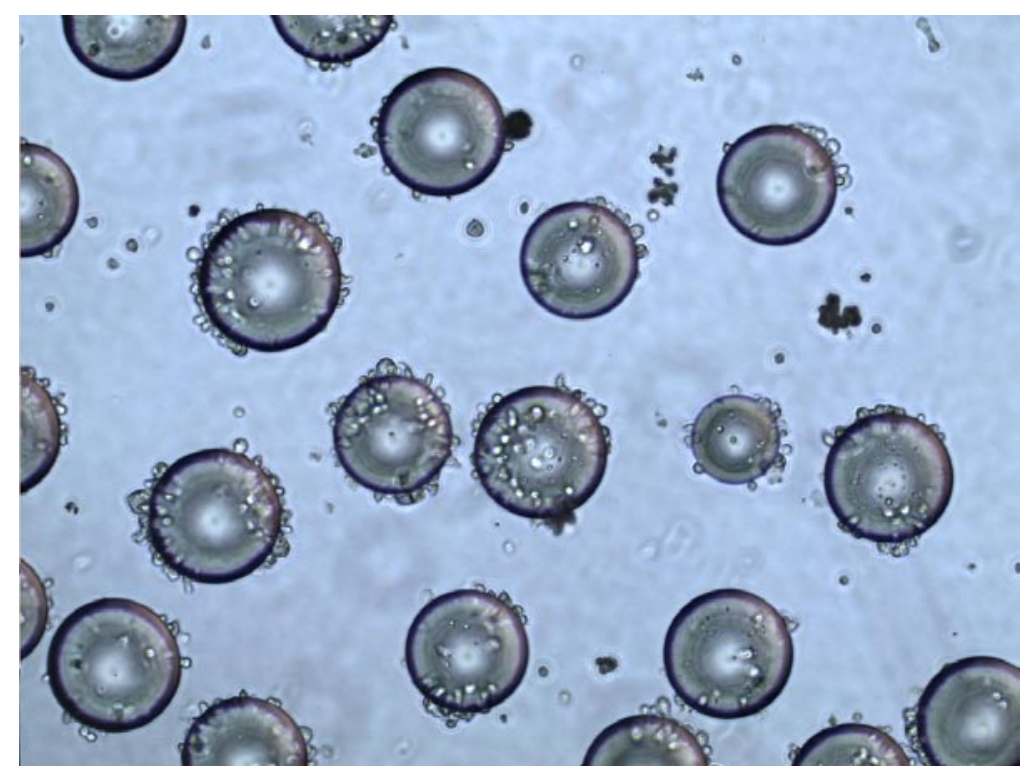

Figure 1.1 Microscopic image of bladder cancer cells binding with smart beads 
Circle detection task has been a very important topic in image processing field and has a lot of applications in real world. In this study, a Hough Transform variation integrated with gradient information is developed to overcome slow processing speed due to regular Hough Transform's huge amount of data in the parameter space. Also, a real valued Artificial Immune System (AIS) based multi-circle detection algorithm is designed as a new stochastic approach. Inspired by biological immune system's distributed structure and ability to recognize and destroy foreign antigens, this real valued AIS based algorithm shows very good performance on precision and robustness for our application. The regular Hough Transform and a geometric feature based circle detection program are also implemented for comparison purpose. Natural image sample from internet is processed by those algorithms as well to show their capability is not limited to this specific beads detection application

This study consists of five chapters. Chapter 2 is literature review part which covers several common circle detection approaches and various AIS models on circle detection problem. Chapter 3 describes the four algorithms of geometric feature based method, regular Hough Transform, modified Hough Transform and AIS based method in more details. Chapter 4 includes statistical results of the complete dataset and samples of beads images and natural images. Chapter 5 presents the advantages and disadvantages of these algorithms, and potential improvements. Appendix A includes result data and Appendix B includes the MATLAB source code for each program. 


\section{CHAPTER 2 LITERATURE REVIEW}

\subsection{Circle Detection}

Circular shape is one of the most commonly seen primitive shapes. Circle detection has been extensively studied in the past decades due to its broad object targeting applications from industrial manufacturing [12][13][14][15], scientific research [17], to biomedical study [16][18][32]. Circle detection fits the most straightforward object recognition method -- template matching [19][20][21], as many other kinds of object detection problems. But template matching fails to make use of circular shape's unique Euclidian geometry property of uniformity and symmetry -- hence it is very sensitive to noises and deformation unless a relative independent and stable feature is applied instead of commonly used contour information.

Usually, in order to take the advantage of circle's uniform shape, several geometric features are utilized in circle detection problem. On the other hand, the well known Hough Transform and its many variation approaches are successfully developed to find circles as well.

Computational Intelligence (CI) represents a series of approaches inspired by biological nature. Recently researches show that Computational Intelligence approaches often outperform traditional methods on solving many real world problems with better adaptability, efficiency and robustness. Therefore, previous applications of Computational Intelligence to circle detection problem are also reviewed in this part. 


\subsubsection{Geometric Feature Based Circle Detection}

A circle is defined as a circumference with all its points equidistant to a fixed center point. The fixed distance is radius, and the center must be inside the circumference. A line segment links any two different points on the circumference is a chord, while the longest chord is diameter.

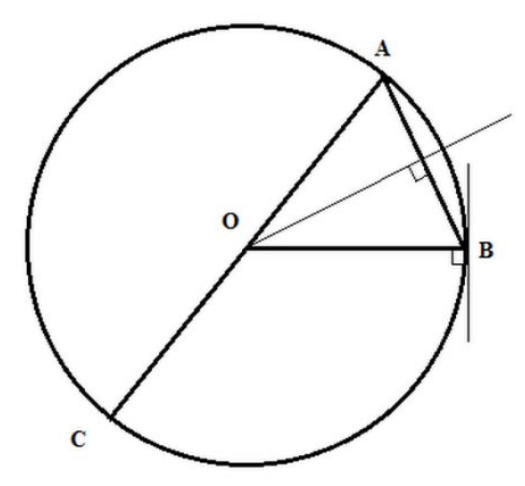

Figure 2.1 Example of a circle

As shown in Figure 2.1, point $\mathrm{O}$ is the center of a circle, line segment $\mathrm{OB}$ is radius and line segment $\mathrm{AC}$ is the diameter. Line segment $\mathrm{AB}$ is a chord, while the corresponding circumference section called arc AB. These properties make circle a very unique shape.

Jia and his colleagues proposed a gradient based method to detect circle centers and radii [22]. As everyone knows, the gradient of a circumference point is perpendicular to the point's tangent and pointing to the center. For instance, in Figure 2.1 vector $\mathrm{BO}$ is the direction of point B's gradient. Jia's method utilizes this attribute, that if lines are drawn along each of the circumference pixel's gradient, the point within circumference which passed by those lines with the most times will be the circle's center. 
In order to implement this, the edge map of an image is obtained first by using Canny edge detection [23], and then each edge pixel's gradient is calculated by a Gaussian template. An energy map with the same size as original image is initialized and used to count straight lines' crossing times at each pixel location. The centers of circles in an image can be found by searching for local maxima after accumulating all lines coincided with gradients. Finally, corresponding radius is found by detecting the location where the total gradient magnitudes on an expanding circumference reached maximum.

The idea of gradient vector is in fact the same idea that the center of a circle must lie on the normal at the edge point used in another paper [24]. However, when there are noises or imperfect shape appearance, gradient information will become erroneous the detection result will be corrupted.

Another geometry characteristic approach proposed by Shang and others is that the hypotenuse of a right triangle inscribed in a circle must pass the center of that circle $[25][26]$.

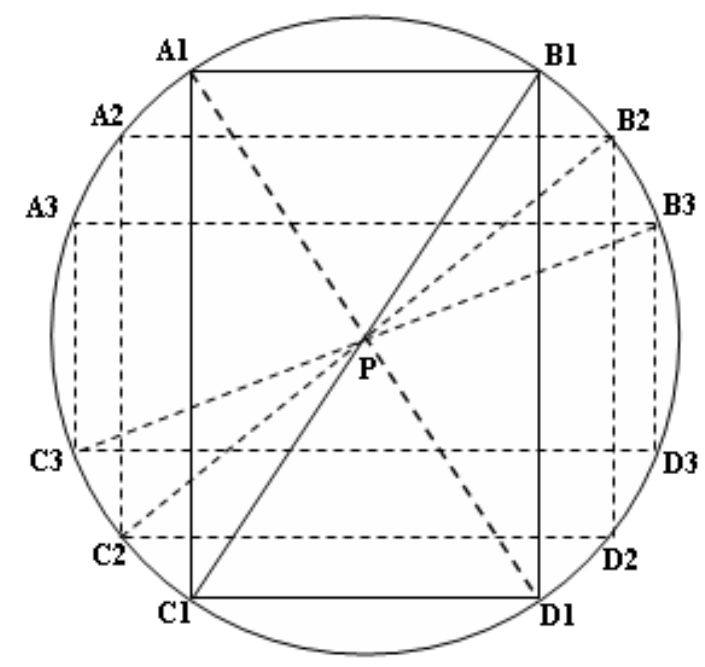

Figure 2.2 Schematic of semi-random search method [25] 
Based on an edge detection step, any three points of a four point-combination A1B1C1 or A1B1D1 on the circumference could define a right triangle. The circle's radius is estimated via the hypotenuse length of the right triangle. The real circles are sorted out through a series of thresholds with empirical parameter setting called semirandom search. To be noticed, the semi-random search is constraint with a predefined repetition limit, and only those edge segments which have at least one pixel with full 255 intensity values are selected for processing. This would require that the circle object must have relative sharp contrast over image background if need to be detected, and made it not very tolerant to real world illumination conditions.

Back to Figure 2.1, it indicates that the perpendicular bisector of chord AB must pass through the circle center. If there are two different already known chords, the center can be located with the help of some trigonometric functions. Kim implemented this idea via a $2 \mathrm{D}$ histogram for locating center followed by a $1 \mathrm{D}$ histogram for searching radius [27], which is a very similar structure to regular Hough Transform as discussed in 2.1.2.

In summary, geometric feature based multi-circle detection is one of the simplest means. But their performance heavily relies on the perfect appearance of circular shape which is hard to guarantee in real world application, and undermines the confidence of detection result.

\subsubsection{Hough Transform Based Circle Detection}

Hough Transform (HT) is initially introduced in the form as a patent [28], and then further expanded into a general method to detect shapes from binary images [29][30]. 
The key idea of Hough Transform is to convert a relative difficult global detection problem in image space to an easier local peak detection problem in parameter space.
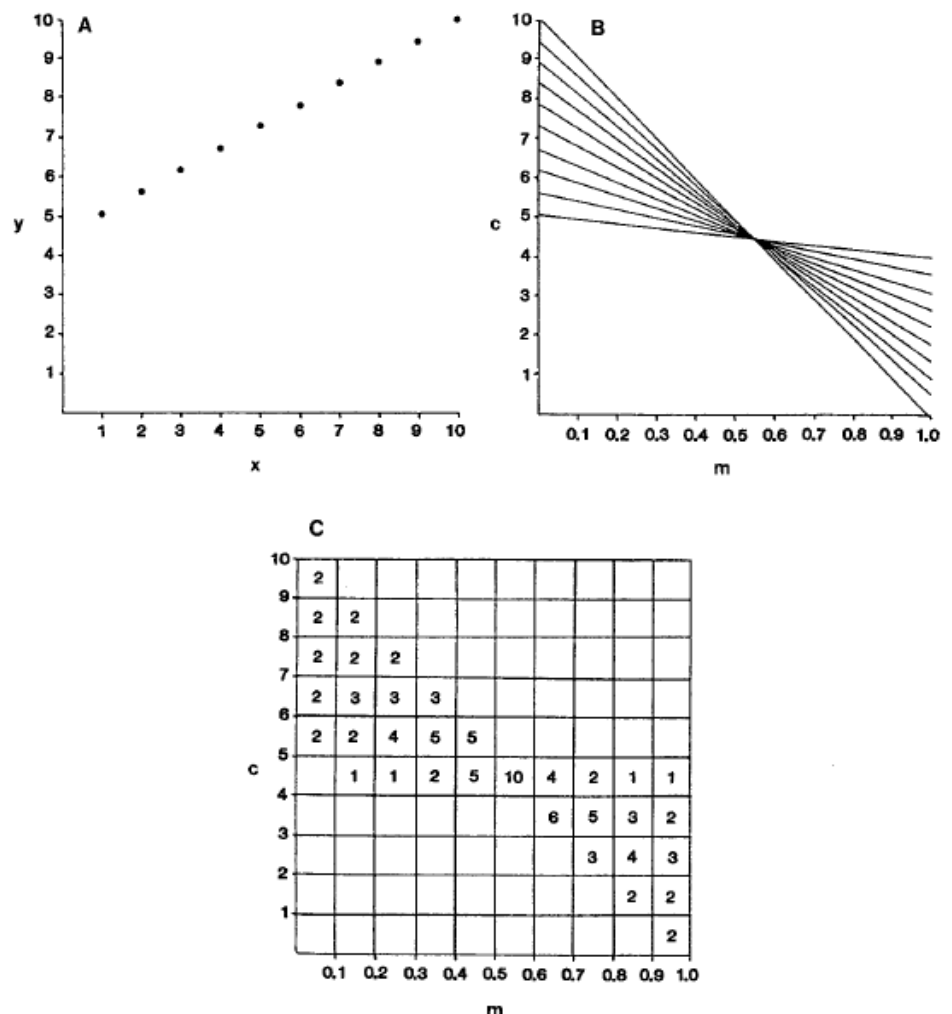

(A) image space, (B) parameter space, (C) accumulator space

Figure 2.3 Example of straight line Hough Transform [31]

Straight line Hough Transform is the simplest example as illustrated in Figure 2.3, the line function in image space is defined by slope $m$ and intercept $c$ in the $x y$ plane,

$$
y=m x+c
$$

Hough Transform considers that this equation could be viewed as a mutual constraint between image space and parameter space. Thus one could convert a straight line in the image space to one point in parameter space (one-to-many projection) by treating $m$ and $c$ as variables and $x$ and $y$ as parameters as in (A) and (B) of Figure 2.3. 


$$
c=-x m+y
$$

Equation 2.2 is a converted version of the same line function. This conversion is also called backprojection mapping[31].

An accumulator plane in (C) of Figure 2.3 with the same size as parameters space works as a matrix of counters to record how many image points are projected to each of the parameter location, or how many lines in parameter space intersect at a same point. When a local peak is formed in parameter space, it means that many image pixels share the same corresponding parameter combination. In other words, they compose of the same detected straight line. By now, the line feature detection problem is converted to a peak finding problem.

Hough Transform can be considered as an evidence voting procedure [31]. Each pixel votes independently in parameter space, which makes it working very well under noisy environment, because irrelevant pixels cannot directly affect this feature voting.

It is a 2-parameter space for a line detection using Hough Transform done in a 2D parameter plane. When extending Hough Transform to more complicated shapes detection, it is called Generalized Hough Transform [34], and the parameter space is expanded to $n$ dimension for arbitrary shapes with $n$ parameters needed to define them. For instance, to detect circles there would be 3 parameter needed -- the coordinates of center $(a, b)$ and radius $r$, or 5 parameters for eclipse shapes. The effort of Hough Transform is determining which point in the $n$ dimensional hyperspace has been passed most times by the surface generated by those shape feature parameters. 


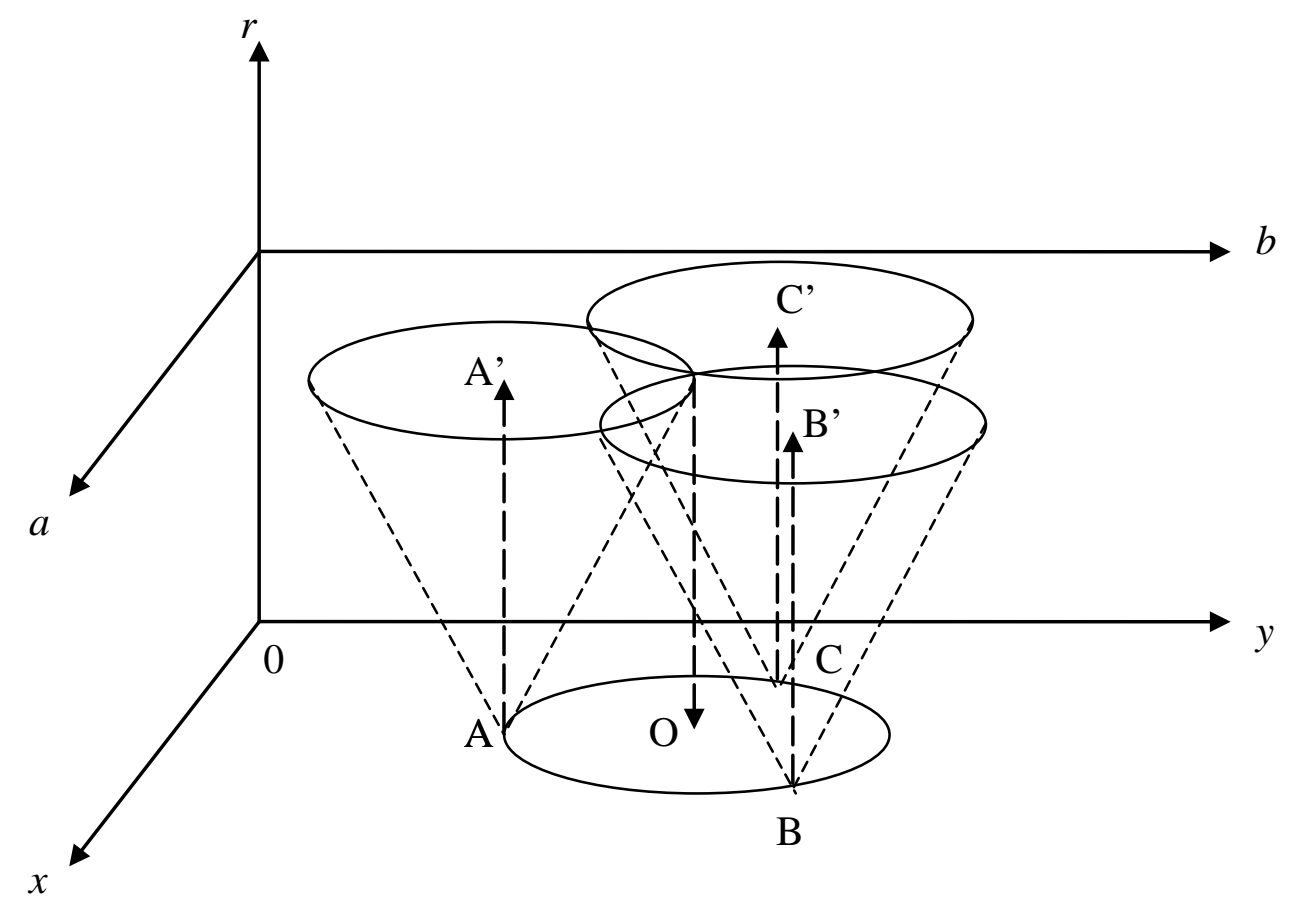

Figure 2.4 Example of circular Hough Transform

Figure 2.4 shows the normal circular Hough Transform with the mapping from image space to parameter space and backward. Point A, B and C are circumference pixels in image space, and A', B' and C' are their projection in the parameter space. Circle A', B' and C' with same radius $r$ as in image space intersected at the same point which are backprojected to real center point $\mathrm{O}$. The reason was the circle function of Equation 2.3 is in the form of sum of squares,

$$
(x-a)^{2}+(y-b)^{2}=r^{2}
$$

When a circumference pixel in $x y$ image plane is projected to $a b$ parameter plane, it becomes the center of a circle in the parameter plane for a fixed value of $r$. Note that in Equation 2.4 where $(a, b)$ are circumference coordinates still is a circle function on the whole. 


$$
(a-x)^{2}+(b-y)^{2}=r^{2}
$$

If the radius $r$ is fixed at first, all the circles in parameter space defined by circumference pixels will intersect at the same point which represents the center in image space. It could be visualized by drawing parameter circles with centers along the real circle's circumference and when with the correct radius, this real circle's center would be passed through for the most times by all the parameter circles drawn. If the passing time is accumulated on a parameter plane like previous straight line Hough Transform, then by searching those high peaks which means more passing times, center location in image space can be found accordingly.

In multi-circle situation, the radii are unknown and thus became the third independent variable. The common method is to stack all the 2D parameter planes each with a different radius value in ascending order, and then the whole parameter space would be looked like a 3D cube as in Figure 2.4. Searching through all the 2D layers to find where maximum peaks appear would give the center location and radius. It is rational to expect that if there are multiple circles with different radius values in an image, the corresponding 3D parameter space will be huge and very hard to process. The regular circular Hough Transform suffers large storage space requirement and slow processing speed.

Illingworth and Kittler did a survey that covered numerous efforts devoted to improve and apply regular Hough Transform [31]. Most papers suggest applying a small accumulation array with varying quantization for parameter space accumulators, which depends on the accuracy required for detection of parameters. Thus the parameters are resolved iteratively in a "coarse to fine” form [35][36]. It could significantly reduce 
parameter storage space especially when computer hardware is very limited decades earlier.

Kimme and Ballard's circle Hough Transform only accumulate portion of the parameter points when their corresponding orientation are within some range [32]. Visually certain arcs are drawn in the parameter space instead of complete circles. The effect is to reduce the data in parameter space to save computation during local maxima finding.

Atherton and Kerbyson implemented circular Hough Transform by using edge gradients and considering radius simultaneously. Straight line segments pointing away from edge points to center are accumulated in parameter space rather than circles as in normal circular Hough Transform [37][38]. It is further modified by combing orientation and distance together into a complex vector format [39] and formulating Hough Transform by one or more convolution operations [40] for the purpose of facilitating computation.

For the same purpose of reducing memory size, Yip achieved circular Hough Transform from a totally different way. The method alters circular shape representation in its original image space, by linearly approximating a circumference with some straight line segments like fitting a polygon on a circle. Only 4 pairs of line segments vectors are needed for parameterization and circle detection, which makes the algorithm significantly simpler than regular circle Hough Transform [41].

Illingworth and other researchers had done plenty of work in comparing different improvements to normal Hough Transform, including various parameter reduction or different parameter accumulator structures, all for the same purpose of reducing memory 
requirement and improving computation efficiency [42][43]. However, so far there is no conclusion of a best solution, and it is not always the case that sophisticated Hough Transform variations will outperform straight forward methods.

Some other researchers combined regular Hough Transform with other algorithms for improvement. For instance, Ioannou combined Hough Transform with geometric property of chord's bisection [44]. The famous Randomized Hough Transform only calculates a few points with the constraint from certain geometric properties of objective shapes [45]. Tooei adapted covariance matrix and K-means clustering to Hough Transform to detect circles [46].

Overall, Hough Transform has been proven to be the best and most popular deterministic method on circular shape detection problems by its unique feature projection and accumulating operations. Its limitation is the heavy computational load which restricts it from applications that require fast processing.

\subsubsection{Computational Intelligence Based Circle Detection}

Computational Intelligence (CI) is a set of nature-inspired computational methodologies and approaches to address complex problems from the real world to which traditional methodologies and approaches are either ineffective or infeasible [47]. It is a relative new research field and its category is still expanding with new concepts and models emerging. This is part of the reason that Computational Intelligence based applications to circle detection problem are not yet widely studied comparing to traditional method like Hough Transform. Without going into too much detail of every existing Computational 
Intelligence models, only those whose applications to circle detection problem have been approved to be effective are discussed next.

\section{Artificial Neural Network}

Artificial Neural Networks simulate the structure and function of a real human brain. One of the most widely used feedforward artificial neural network model is multilayer perceptron, which consists of at least three layers of artificial neurons embedded with some activation functions. And all these neurons are interconnected with adjustable weight coefficients. A supervised learning process through backpropagation is required to generalize certain classification ability before a neural network can work properly just like the learning and doing process of a real human.

Multilayer perceptron has been proved to be an effective and relative easy way to implement in many pattern recognition problems. However, it has a perquisite of applying supervised learning, which is that some predefined positive and negative pattern data samples are needed for the training process. This can be a shortcoming of artificial neural network comparing to other self-organizing algorithms for some problems where negative patterns are rare or even not available. In addition, due to the nature of a fixed network structure, it is not very flexible to apply to multi-circle detection problem due to the variation in object size but a fixed size input layer. Therefore, only a couple papers focus on fixing artificial neural networks to circular shape detection.

In a coin recognition system, the circular coin image is scanned and then cropped out from background at first and further compressed to a size of 20-by-20, to feed into the neural network for coin classification [48]. Another example of neural network's 
application involves circular shape detection is a cell detection system. In fact the neural network is only responsible for cell feature classification, while the location of the circular cells in the image is solved by Hough Transform. Detected circular areas are compressed to size of 16-by-16 for multilayer perceptron processing [49].

It should be concluded that a step of unifying input patterns sizes is necessary when applying artificial neural networks, and makes it not flexible when circular shapes from real natural images may be with very different diameters. Though artificial neural network can be a powerful classifier in some other pattern recognition task, its limitation in the input data type heavily restricts its performance for circular shape detection

\section{Swarm Intelligence}

Swarm Intelligence is inspired by natural swarm flocks' behavior. It is a collection of models which simulate the distributed and self-organized individuals of a group population. Swarm Intelligence models are typically made of a number of individuals with some simple interaction abilities among themselves and toward outside environment, but show certain functional global behavior which can be utilized to solve complicated problems. There are some models of Swarm Intelligence effectively adapted to circle detection problem.

Ant Colony Algorithm is a probabilistic optimization method. It mimics ant's colony behavior of finding the shortest path for food. Even though at beginning each ant would be searching at random directions, eventually they converge to the shortest path by strengthening shorter paths and abandoning longer ones through communications. 
Chattopadhyay's circle detection scheme applied Ant Colony Algorithm and developed a modified version with Ant Regeneration and Recombination [50]. In this approach, Ant Colony Algorithm is used to detect closed edge loops through traveling edges by ant agents, the final circle detection criterion is to threshold the deviation error derived from the detected radius which the distance between corresponding edge pixels and circle center. This method is essentially a close loop tracking, and its performance is questionable when circular shape edges are not enclosed.

Bacterial Foraging Algorithm is another decentralized model imitates natural bacteria proliferation. Its adaptability is achieved by simulating the phenomenon that bacteria can direct their movements according to certain chemicals in the environment called chemotaxis.

Dasgupta proposed an Adaptive Bacterial Foraging Algorithm with adaptive chemotactic step size which helps it to avoid oscillating around optima and converge faster [51]. The Adaptive Bacterial Foraging circle detection uses a number of evenly distributed points from circle circumference as bacterium representation, which also help to increase its ability to detect incomplete circles than randomly spread individuals over the searching space. It is indicated that this Adaptive Bacterial Foraging Algorithm outperform classical Genetic Algorithm. However, it is not inherently capable of detecting multiple circles. In order to detect a number of circles, the algorithm needs to be repeated and mask out detected circle for each run until a pre-fixed number of times is reached. Then all detected circles are double checked by comparing connectivity with real edge points, to sort out non-existing false detection. Because each run is already 
made of many proliferation iterations and only returns one circle, a multi-circle detection could require a much longer time depending on the number of object circles in the image.

Artificial Bee Colony Algorithm is a much newer Swarm Intelligence model appeared in 2005 [53], comparing to Ant Colony Algorithm. Though the agents in population are both insects, the difference between Ant Colony Algorithm and Artificial Bee Colony Algorithm is the latter further divides agents group into three different types, each with a different function such as workers, onlookers and scouts, where individuals in Ant Colony Algorithm are defined identical.

Cuevas applied Artificial Bee Colony algorithm to circle detection in images, and additional memories are used for storing and checking other potential local maxima for multiple circles condition, which works after the global optimum is found as the primary circle in field of view [52]. For multi-circle finding, the additional memories consist of all previous iterations are first rearranged in descending order according to their matching function, then each memory layer's maxima are compared and grouped, maxima below a distinctiveness threshold are grouped and considered to be one circle. This method potentially needs a lot of memory space if the iteration is set to a large number, but it saves rerun computations comparing to Artificial Bee Colony based method.

Swarm intelligence shows much better adaptability than artificial neural networks and relative good test result. However, it is not inherently capable of multi-circle detection. Most programs need large memory space to keep processing record for multicircle retrieval.

\section{Genetic Algorithm}


Genetic Algorithm has a history over 50 years which makes it one of the oldest Computational Intelligence algorithm series and one of the best developed algorithms both theoretically and practically. It inherits Darwin's famous "Survival of the fittest" natural selection theory.

The individuals in Genetic Algorithm model are called chromosomes or genes, usually presented by binary strings with a fixed length of 0s and 1s. A fitness evaluation scheme is also incorporated to simulate the natural selection of fittest individuals.

During each generation through the evolution iterations, a number of individuals are selected stochastically but also based on their good fitness, to combine to generate the new generation by switching bits segments. The child generation would replace older ones to keep a stable size of the entire population. Some other chromosomes are chosen to mutate at a lower probability by flipping bits, to formulate completely new patterns. The evolution process of crossover and mutation take place over and over, until a number of iterations or some fitness level is reached. According to Darwin's theory, through the evolution process, the global fitness level is continuously improved and eventually the fittest to that environment would appear and spread.

Genetic Algorithm has a much longer history in solving circle detection problem than any other Computational Intelligent methods. The first influential paper was published in 1992 by Roth and Levine [59]. The chromosome is defined as a minimal subset, which is the smallest number of point required to define a primitive shape. A fixed size shape template is derived from chromosome to check the data points in image. Theoretically, this method could detect any shapes defined by a limit number of points. But this paper did not go into the details of any specific application. 
In 1995 Kawanishi and Masafumi designed a genetic algorithm based shape detection method, which is capable of detect primitive shapes like lines, circles and ellipses as well [54]. The chromosome uses center and radius information to define circle circumference. The key feature of this method is to defocus the single pixel edges with a 5-by-5 kernel and transform the binary edge map into multi-value search space. The 5pixel wide edges would give genetic template a direction to evolve, while the matching ratio of a thin shape and the genetic template could only reach a peak value within a very narrow range. If at 1-pixel width level, it would be very difficult to determine how close the matching was.

A couple years later, Yin improved the genetic algorithm approach by quantizing the matching template to a fixed length of 360 pixels in 128-by-128 image for fitness evaluation. The improvement includes introducing lookup table by using the symmetrical property of circle shape to reduce the computation, and adding a candidate reselection to find best fitted circles after genetic algorithm phase. This method also could detection more than one shape in an image [55]. The shortcoming of this method is the low resolution of images, especially when there are many circles and other shapes, the number of pixels which can be used to discriminate the difference between each of the targets became very limit and could deteriorate the performance.

Another genetic algorithm based circle detection used the coordinates of random three edge points to represent chromosomes instead of using center coordinates and radius combination as usual [56]. This representation unified the data to pixel level, in contrast that a radius was not directly represented in image space. 
Its evolution operations include roulette selection, bitwise crossover and mutation. Fitness of chromosome is evaluated by an existence ratio of a number of edge points derived from the 3-point combination of chromosome. Similar to Dasgupta’s method [51], multi-circle detection was achieved by setting an anticipated number of objects, and rerun the program and mask out detected circle each time.

Karkavitsas and Rangoussi's method is particularly designed to detect blood cells in microscopic pictures [57]. One major difference of this application is that a segmented image is used as search space instead of edge image, with binary chromosome encoding of center coordinates and radius. In such case, the fitness is defined in a different way, as an area overlapping ratio between circles and segmented cell blobs instead of strict edge points overlapping. It also results in that the stop criterion is detection coverage lower than $40 \%$, since at last most chromosome circles converge to certain segmented area.

Simulated annealing is another member of Computational Intelligence, an analogy of the annealing technique from metallurgy. It is efficient to handle global optimization problem when search in very large data set. Das's automatic circle detection method integrated simulated annealing with Differential Evolution [58]. Simulated Annealing is modified to perform the selection mechanism of Differential Evolution. Population representation is in the regular center and radius form. Although this method is robust to noise, it fails to detect circle locations in a sense of precision, under both clear and noisy conditions seen from their result samples.

In summary, the Genetic Algorithm is the most favored computational intelligence model for multi-circle detection so far, and has been proven to be more suitable for multi-circle detection problem among other computational intelligence based 
methods. However, due to the nature of global optimization of Genetic Algorithm, multicircle detection required additional processing. The ideal case would be an algorithm has the adaptability and robustness like computational intelligence model, and only need to run once like regular deterministic approaches.

\subsection{Artificial Immune System}

Artificial Immune System (AIS) is a newly emerged member of Computational Intelligence family. Because of the natural immune system's biological importance, it is born to be extremely complex and involves a lot of operations. By understanding natural immune system's functions, powerful tools could be derived from this autonomous system and used to solve complicated real world problems [60].

In the following part, the basic immunology principles are introduced and some typical Artificial Immune System models are discussed, for the purpose of exploring the feasibility to apply Artificial Immune System to our multi-circle detection problem.

\subsubsection{Natural Immune System Principles}

Mammals have a robust defense system called the immune system to protect themselves from foreign and potentially harmful substances called antigens. The immune system has a complex structure consists of different molecules, cells and organs. They coordinate

and response as an organized system to defense against dangerous substances, like bacteria, viruses and parasites. In general, natural immune system has very strong information processing ability to detect non-self dangerous elements and to give 
appropriate protective response, but tolerating many harmless substances at the same time.

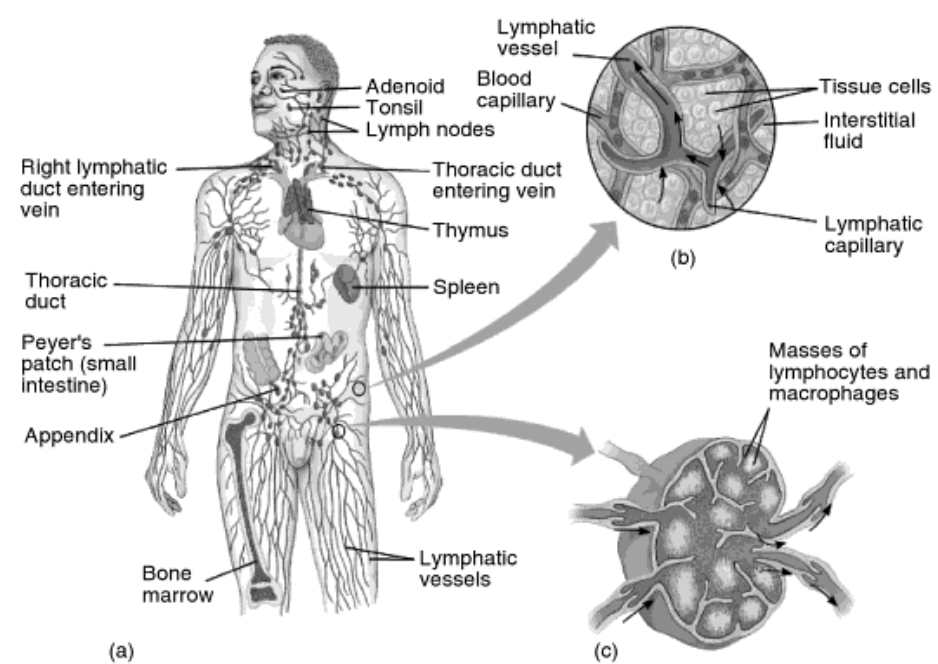

Figure 2.5 Functional components of human immune system [61]

Taking human immune system as an example in Figure 2.5, the major organs include bone marrow, thymus, spleen and lymph node. Among them, the bone marrow and thymus are like factories where immune cells are produced and maturated. Spleen and lymph node are like coordination centers where immune cells are filtered and activated.

There are many different types of immune cells throughout the whole immune system. They are the soldiers of this system who directly confronting with foreign antigens. Lymphocytes are the most crucial constituents of this army. They are produced in the bone marrow, circulate the entire body through blood and lymph system, and respond to most immune activities.

The lymphocytes are divided into two types -- $\mathrm{T}$ cells and B cells, and each of them has two sub-types. The T cells could be either killer cells or helper cells. The killer 
T cells kill foreign cells and the infected self-cells as well, while helper T cells help B cells to perform antigen recognition by releasing cytokines. B cells could be activated to become plasma cells which secret antibodies, or to become memory cells to keep the recognition of antigens for a long time for quicker response in the future. Antibodies generated by plasma B cells are a particular kind of proteins which can bind and neutralize antigens [61].

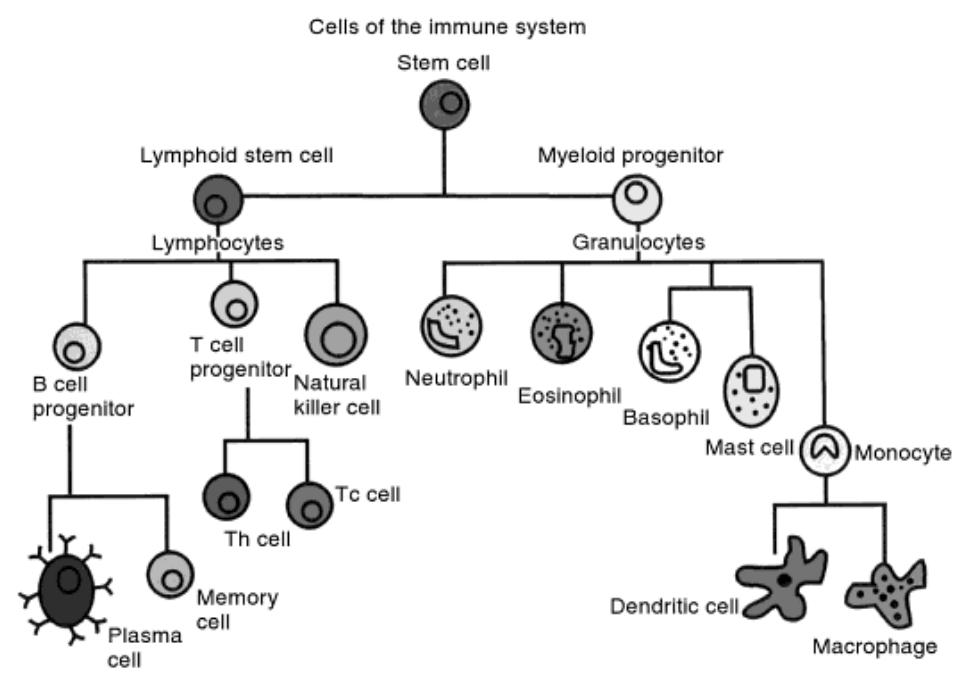

Figure 2.6 Cells of natural immune system [61]

Some researchers prefer considering natural immune system from a multi-layer perspective. In that case, the immune system is divided into four functional layers. They are the skin and mucous membrane as first physical barrier; physical condition like body temperature and fluid $\mathrm{pH}$-value as the second layer; a non-specific innate immune system born with a person as the third layer; and final and most powerful adaptive immunity layer which has the ability to specifically recognize and destroy harmful antigens [62]. 


\subsubsection{Development of Artificial Immune System}

From the perspective of information processing, the natural immune system is a highly parallel and distributed adaptive system. It can learn to recognize relevant patterns, remember those that have been perceived, and construct new pattern detector by combining what it learns. Inspired by the natural immune system’s remarkable capability, several Artificial Immune System models based on the natural immune mechanisms are developed to solve recognition and classification problems. The three major Artificial Immune System models are Immune Network theory, Negative Selection mechanism, and Clonal Selection Algorithm [63][65].

The Immune Network theory is introduced in 1974 [64]. It says that the immune system maintains an idiotypic network of interconnected B cells. The idiotypic network theory is based on the concept that lymphocytes are not isolated, but can communicate with each other through interaction among antibodies. Thus, the recognition of antigens is done at a system level by the sets of interactions of antigen-antibody as a network [66].

The Negative Selection mechanism is proposed by Forrest [67] and is based on T cells' self-nonself discrimination ability. During the generation of $\mathrm{T}$ cells, they goes through a censoring process called negative selection, in the thymus where $\mathrm{T}$ cells that react against self-proteins are destroyed, while those do not bind to self-proteins are allowed to maturate and circulate throughout the body to only bind to foreign antigens [66]. It is called negative selection because randomly generated detectors are screened by

eliminating who respond to predefined targets rather than keeping them for positive selection. 


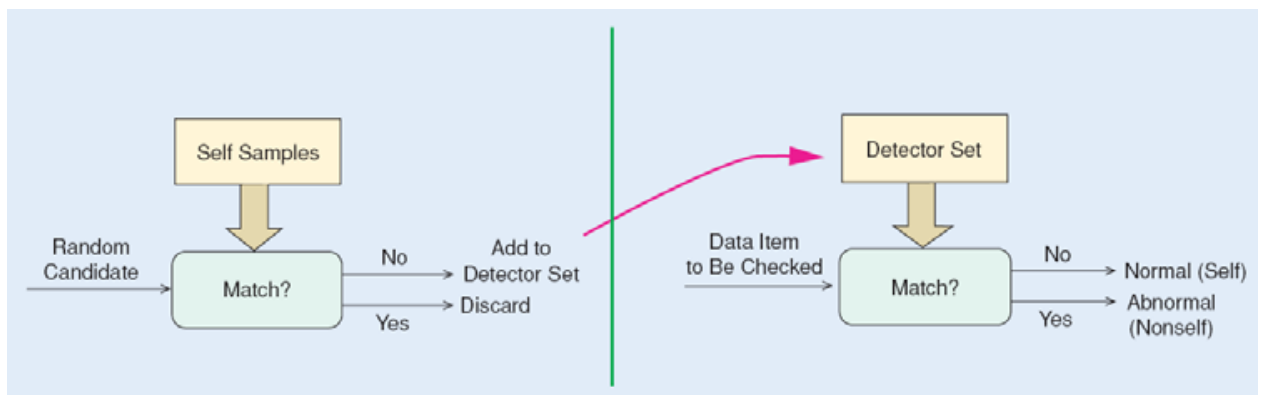

Figure 2.7 Negative Selection principles [65]

The Clonal Selection principle describes the idea that only those B cells that recognize the antigens are selected to proliferate. This selection is subjected to an affinity maturation process, which improves B cells affinity to the selective antigens [68]. The affinity is the binding fitness between antigens and antibodies, and its measurement could be Hamming distance when using binary encoding or Euclidian distance with real valued encoding. 


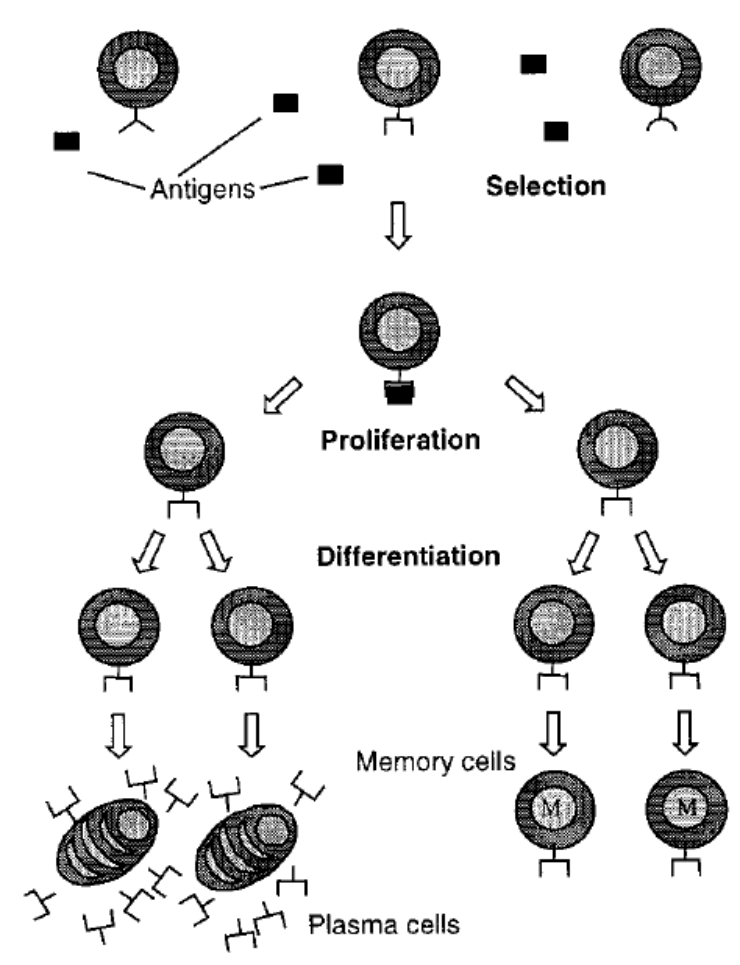

Figure 2.8 Clonal selection principles [68]

The random generation and mutation process in Clonal Selection Algorithm is similar to Genetic Algorithm. However, there are several distinct differences between Genetic Algorithm and Clonal Selection:

1) the population size in Clonal Selection is adjustable while Genetic Algorithms usually use a fixed total number;

2) Clonal Selection does not have a crossover operation, while Genetic Algorithm has;

3) Clonal Selection can solve and maintain multiple local optima solutions, while Genetic Algorithm is more a global optimization.

Many applications of Artificial Immune System were discussed in [63][65][66][69] and are concluded in Table 2-1. 
Table 2-1 Applications of Artificial Immune System

\begin{tabular}{|l|l|l|}
\hline AIS Model & Solving Problem & Application \\
\hline $\begin{array}{l}\text { Immune Network } \\
\text { Theory }\end{array}$ & $\begin{array}{l}\text { Supervised or } \\
\text { unsupervised learning }\end{array}$ & Classification, clustering, data mining \\
\hline $\begin{array}{l}\text { Negative } \\
\text { Selection }\end{array}$ & $\begin{array}{l}\text { Abnormality detection, } \\
\text { damage detection }\end{array}$ & $\begin{array}{l}\text { Computer virus detection, system } \\
\text { process monitoring, fault detection }\end{array}$ \\
\hline $\begin{array}{l}\text { Clonal Selection } \\
\text { Algorithm }\end{array}$ & Searching, optimization & Pattern searching, optimization \\
\hline
\end{tabular}

Despite many successful applications of Artificial Immune System, it is not widely used regarding image processing problems yet. McCoy and Devarajan applied Clonal Selection Algorithm to segment road pixels from IR-sensitive aerial imagery [70]. In contrast to their previous Genetic Algorithm based approach, Clonal Selection Algorithm doesn't suffer the problem of a single detector taking over the population and becoming an evolution dead end. In other words, Clonal Selection Algorithm is multimodal optimization process rather than a unimodal or global optimization.

Other image segmentation applications of AIS use Negative Selection. For instance, Sathyanath applied Negative Selection to the color image classification of wood patterns [73]. Zhou's Negative Selection chose to use real value vector to segment X-ray dental images. Their result shows Negative Selection is more sensitive to detect abnormal cases than Support Vector Machine [71].

Researchers started to explore the potentials of AIS for shape detection very recently. A more theoretical attempt is Isa's shape recognition program with Clonal Selection Algorithm. It works at image frame level instead of pixel level like most common image processing methods. Both the antigens and antibodies are designed as 10by-10 images and representations are binary string. This makes it not very practical to process normal resolution images [72]. 
A more promising work is done by Cuevas et al., which is a circle detection method also based on Clonal Selection Algorithm [74]. The circle candidates are designed as antibodies using 3 circumference points with binary encoding, and real existing circles are treated as antigens. A fitness function is designed to evaluate the matching between candidate circles and real circle pixels. Just like early Genetic Algorithm based method, multi-circle detection is achieved by analyzing memories of previous iterations. High detection rate is achieved among synthetic and real images.

In summary, Artificial Immune System has been developing very fast in the last decade, but not yet widely applied to shape detection in image processing. The successful examples of Artificial Immune System on shape detection have strong connection with Clonal Selection Algorithm. The difficult part of implementation is how to represent data and effectively extract shape features. In chapter 3 a new real valued circular shape detection method is introduced with details, and compared with Cuevas's Clonal Selection method. 


\section{CHAPTER 3 ALGORITHM DEVELOPMENT}

In this chapter, four circle detection algorithms are described in detail, in the sequence of a geometric property based algorithm, the regular Hough Transform, the modified Hough Transform with gradient information, and the real valued Artificial Immune System.

\subsection{Original prototype algorithm}

Smartbead Inc. has developed the original prototype program for the automatic grading system [80]. The system is made up by two steps. The first step is to isolate each individual bead, and the second step is to evaluate the cells binding condition.

The first step of bead detection of this algorithm is based on watershed algorithm [76]. The raw RGB color image with 2048-by-1536 resolution is downsized by $1 / 4$ and converted to 8-bit gray-scale image. The downsize ratio is acquired empirically and represents the best balance of data size between entire image and effective representation of objects. Then the gray image is complemented, and its gradient map is computed and thresholded. Because most beads in image have a darker contour, followed by morphological operation of filling holes, isolated beads would become white blobs. These blobs are used as starting seeds for watershed by flooding to detect real beads edges. Watershed segmentation result is filtered again according to object's size and aspect ratio to improve results confidence.

After beads detection is finished, the algorithm thresholds the gradient map at a

different level to segment cells for second step. Only cells on and within beads contour are analyzed for grading. 
The advantage of this algorithm is that it only applies simple image processing computation like gradient, threshold, and watershed segmentation, thus it processes image very fast. The disadvantage is that there are many empirical values which are very sensitive to different image conditions and need to be adjusted accordingly, such as multiple structuring elements in morphological operation, segmentation threshold and size limits used to filter false contours. Otherwise the algorithm fails frequently. For example, the regions where watershed algorithm starts are based on regional local maxima, but if previous morphological operation cannot help to acquire disconnected maxima regions, watershed algorithm cannot return independent contours either.

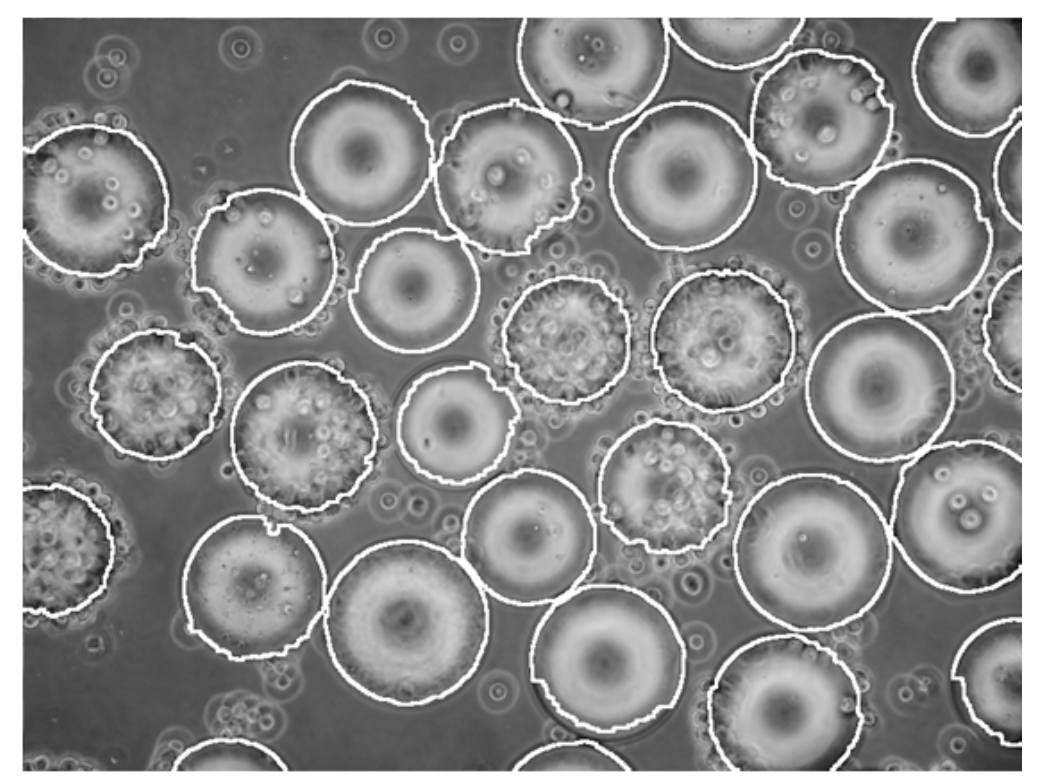

Figure 3.1 Successful example of first prototype algorithm 


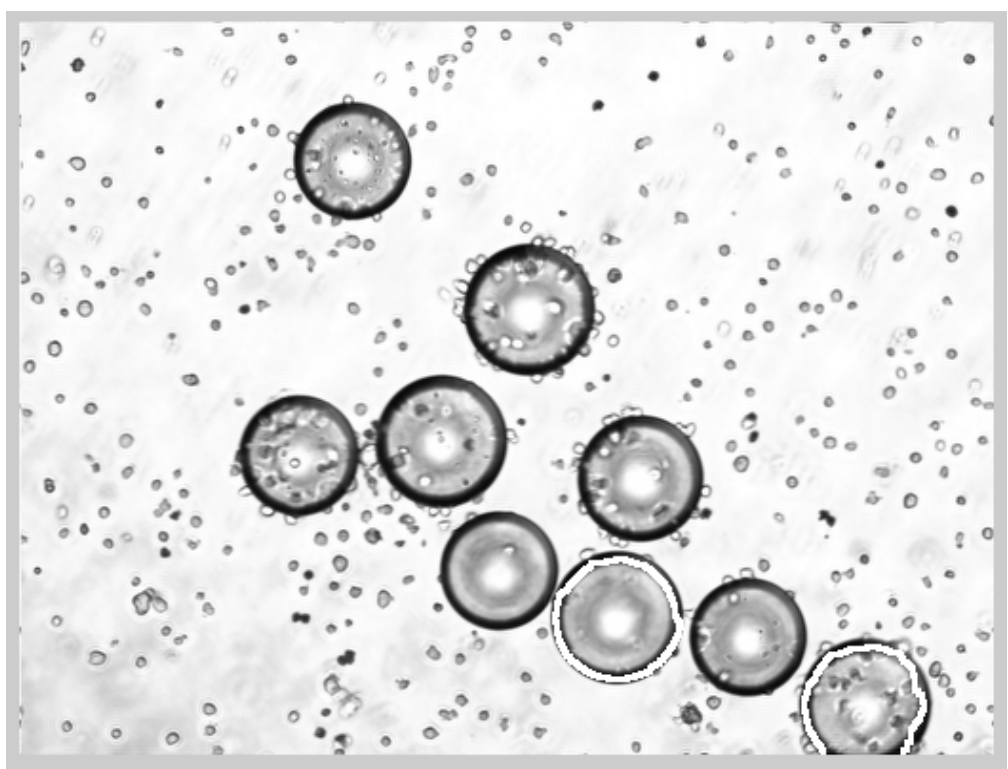

Figure 3.2 Failed example of first prototype algorithm

Figure 3.1 and Figure 3.2 show the performance of first prototype algorithm with unchanged parameter setting. Detected beads are highlighted in white color. It is obvious to see that the detection performance is very unstable and the beads contour detection is inaccurate either. This confirms the necessity to develop a new robust algorithm for better accuracy.

\subsection{Image preprocessing}

The four algorithms developed in this study share the same preprocessing part. In preprocessing, the raw image is processed to reduce redundant data and provide an input edge map for different recognition algorithms.

The raw microscopic image is taken from a digital camera with resolution of 2048-by-1546 and in JPEG format. Edge detection is chosen here instead of color based 
segmentation, because edge information depends on pixel intensity which is more resistant to illumination variations than color appearance.

Some experiments are done trying to use color component based segmentation by thresholding in $\mathrm{YCbCr}$ color space to separate beads from background. The problem is that the images in dataset do not have a consistent illumination condition and color based segmentation frequently fails. An illumination correction process would be more troublesome than edge detection since the images already have a relative uniform background and good contrast thanks to microscope slides.

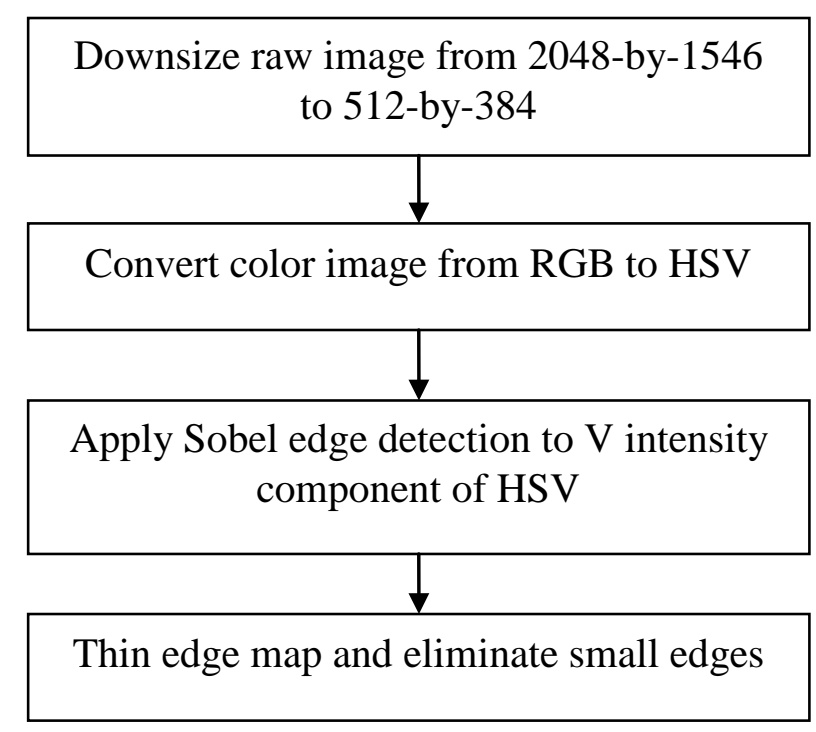

Figure 3.3 Image preprocessing diagram

A decimation operation is applied first to reduce the size of raw image to reduce computation with lower resolution but still with enough pixels for feature extraction. The decimation ratio of $1 / 4$ same as Smartbead Inc.'s prototype program is used.

HSV stands for hue, saturation, and value(or intensity) [81], where the intensity component is used for edge detection. The reason is that these three components are 
relative independent than RGB color space of which three components all have intensity information and correlated, influenced by lighting condition.

The Sobel edge detector is a kind of first order derivative operation. It is made of two 3-by-3 window to digitally approximate partial derivatives in vertical and horizontal directions. The gradient magnitude and orientation can be calculated through 2D convolution of the Sobel kernel and intensity image by the following equations (3.1) (3.4) [76]. It should be noted that in image processing, the origin is always the top left corner of image frame, and row direction is represented by $\mathrm{x}$ as vertical axis, while column direction is represented by y as horizontal axis, which is a rotated version of regular Cartesian coordinate system.

$$
\begin{aligned}
& \text { GradX }=\left[\begin{array}{ccc}
-1 & -2 & -1 \\
0 & 0 & 0 \\
1 & 2 & 1
\end{array}\right] \\
& \text { GradY }=\left[\begin{array}{ccc}
-1 & 0 & 1 \\
-2 & 0 & 2 \\
-1 & 0 & 1
\end{array}\right] \\
& \text { GradMag }=\sqrt{\text { Gradx }{ }^{2}+\text { Grady }^{2}} \\
& \text { GradAng }=\operatorname{atan}\left(\frac{\text { Grady }}{\text { Gradx }}\right)
\end{aligned}
$$

The advantage of the raw image dataset in this study is the relative uniform background with only normal cells and debris scattered around, thus Sobel edge detection with a magnitude threshold is better than Canny edge detection which gives too much irrelevant edge segments from cells and beads textures. This is chosen by trial and error.

In order to further reduce redundant pixel points, a thinning operation is applied [76] to reduce edge segments to single pixel wide, followed by a process that eliminates all the edge segments smaller than $10 \%$ of image height. Because any object smaller than 
this size cannot be bead target for this study, and eliminating them helps reducing computational load for following steps. The final edge map is very clean with enough information for further circle detection.

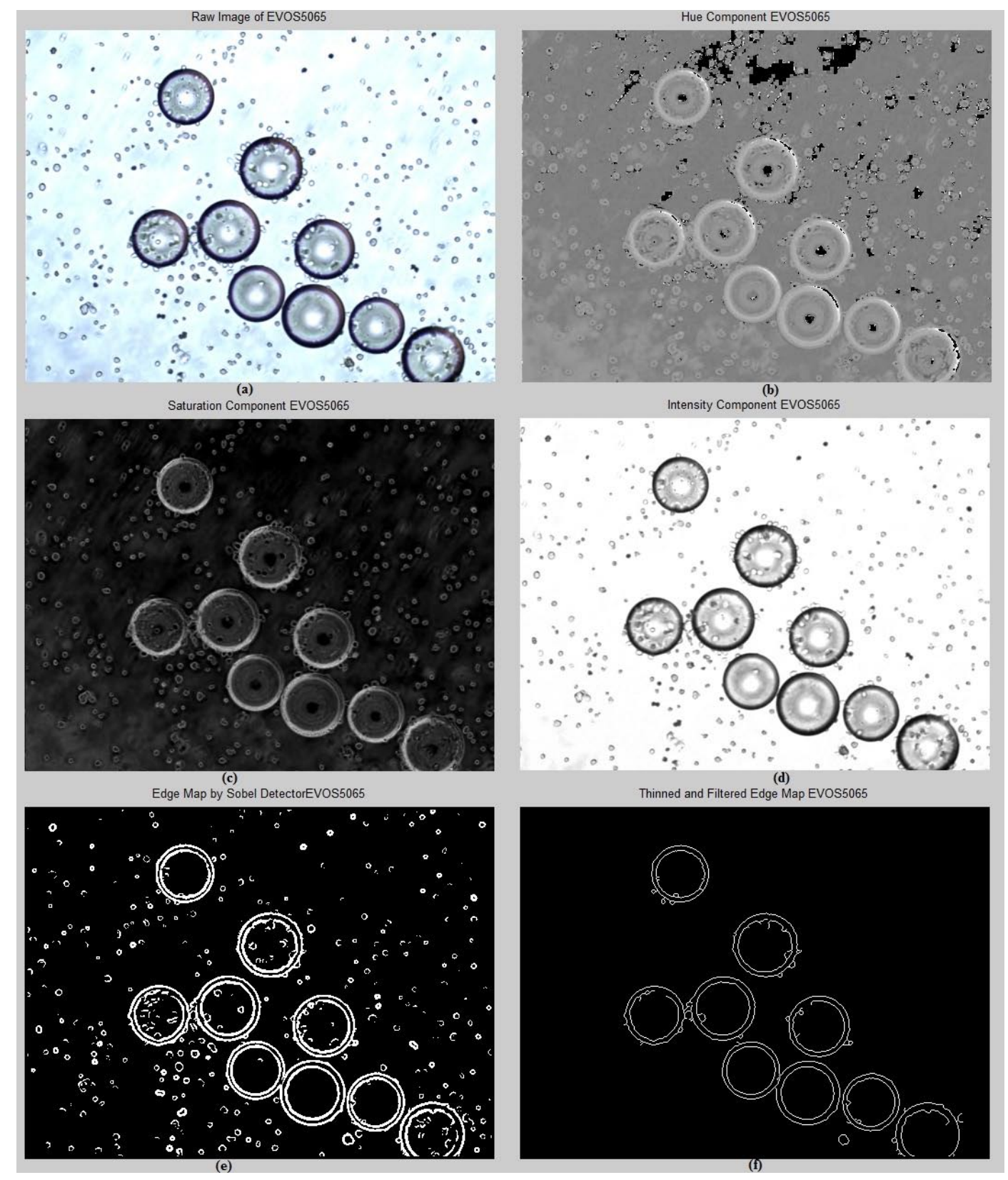

(a) Raw image \#5065, (b) hue component, (c) saturation component, (d) intensity component, (e) edge detection by Sobel kernel, (f) thinned and filtered edge map

Figure 3.4 Examples of image processing 


\subsection{Geometric property based algorithm}

This geometric property based algorithm is based on Jia's paper [22] with three major modifications to make it more suitable for this study. The main advantage of this method is that it requires much less memory than Hough Transform, relatively simple to implement, and relatively good performance.

First, a single pixel wide straight line along each circle’s edge point’s gradient direction is drawn and passed through the corresponding center of this circle like discussed in 2.1.1. In the original paper, 3-pixel wide lines are used. Single pixel width is chosen in this study for a better location precision. If stack all those lines derived from edge points in an image on a black background named energy map, and each coordinate position on this energy map works as a counter which counts how many lines pass through them. Then after drawing all lines, the counter which accumulates a high energy peak would be a possible center location.

Secondly, to estimate the radius of each detected center, starting from an initial circular region defined by center $\left(x_{0}, y_{0}\right)$ and a radius value $R$, the summation all edge circumference points' intensity from Figure 3.4 (f) of this circle is calculated repeatedly when $R$ increases from lower limit to a higher limit. It is expected that the true circle radius is the corresponding $R$ where the gradient magnitude summation reached its maximum value as $R$ increasing from the lower limit to higher limit. In the original paper, it sum the gradients of all circumference points with variable $R$. Since circumference points' intensity is either 1 or 0 in thinned and filter edge map, the summation computation here is easier than in the original paper where calculates original floating 
point gradients derived from equation (3.3). A histogram of circumference pixel summation is used to estimate radius by finding maximum summation value.

Third, the peak finding procedure is done in a recursive way. First set a minimum peak limit equals to $1 / 3$ of the first global maximum peak value, obtained by trial and error. Then, repeat the steps of finding global maximum and then setting its surrounding area to zero, until the global maximum is smaller than minimum limit. In the original paper, 2D order-statistic filter is applied first to find local maxima regions first, then more accurate center location is found by finding the point with energy level equal to regional maxima.

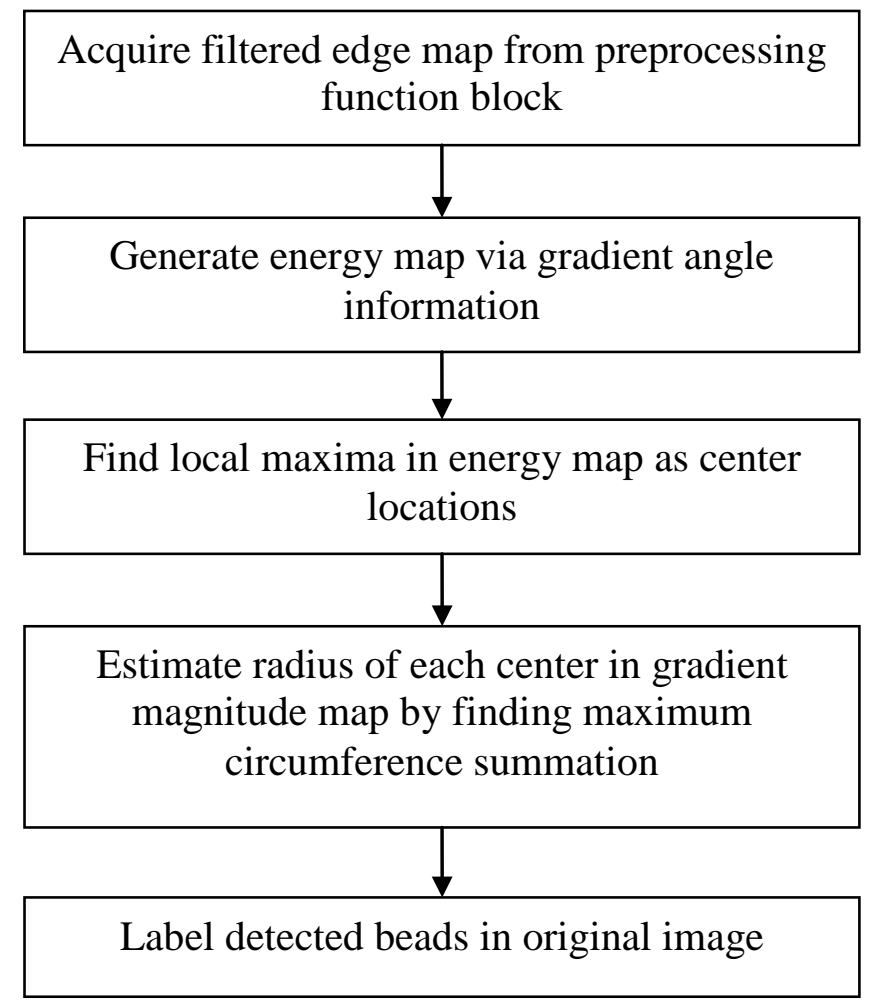

Figure 3.5 Function block diagram of geometric based circle detection algorithm 

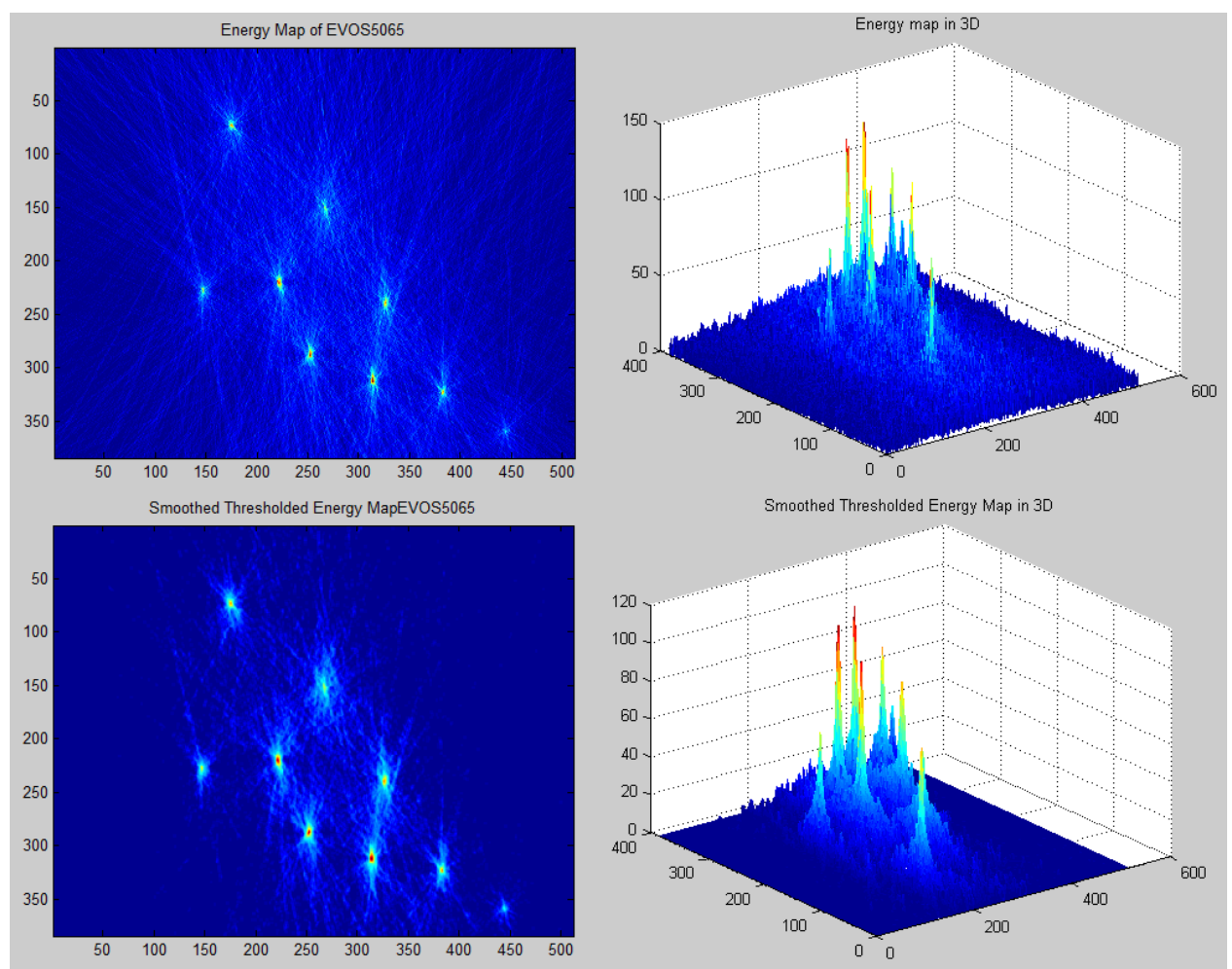

Figure 3.6 Energy map with line accumulation

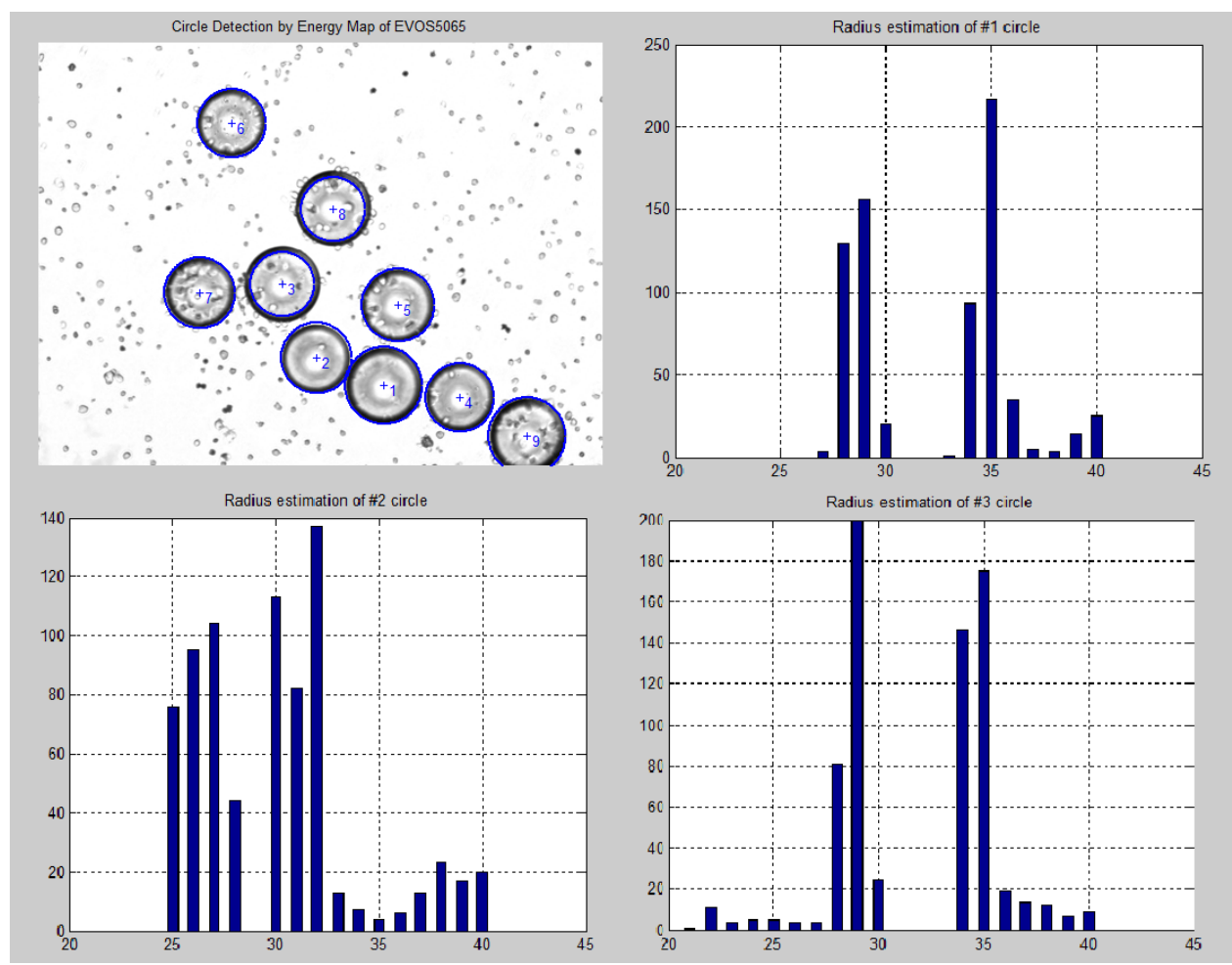

Figure 3.7 Final detected beads and radius histogram examples 
The first generated energy map generated by the histogram of gradient oriented lines then goes through a suppression operation with a threshold $\mathrm{T}$ associated with energy map's mean and standard deviation, followed by an average filtering for smoothing. The suppression operation sets any point's energy below $\mathrm{T}$ to 0 , which would improve peak finding performance.

$$
T=\operatorname{mean}(\text { energy })+2 \cdot \operatorname{stdev}(\text { energy })
$$

From Figure 3.7 it is easy to spot final detection in upper left image, bead's centers are labeled with a blue cross and numbered, and circumferences are highlighted in blue as well. The radius estimation histogram examples are for beads \#1, \#2, and \#3. Their radii are found to be 35, 33, and 29 respectively, which are all correct.

One shortcoming of this method resides in generating energy map, because the minimum peak limit is relatively fixed, for different images, smaller sized beads might not be able to accumulate high enough peaks to get picked out, while other beads with non-perfect edge shape which provide wrong gradient direction and form local maxima away from real center. And this would further affect radius searching because that is based on the round symmetrical property of circle. The advantage obviously is the simple energy map as 2D searching space and relative fast processing speed.

\subsection{Regular Hough Transform}

The regular Hough Transform is introduced in 2.1.2 in advance. Since in multiple circle detection the radius information is unknown, a 3D parameter space is necessary for implementation. 


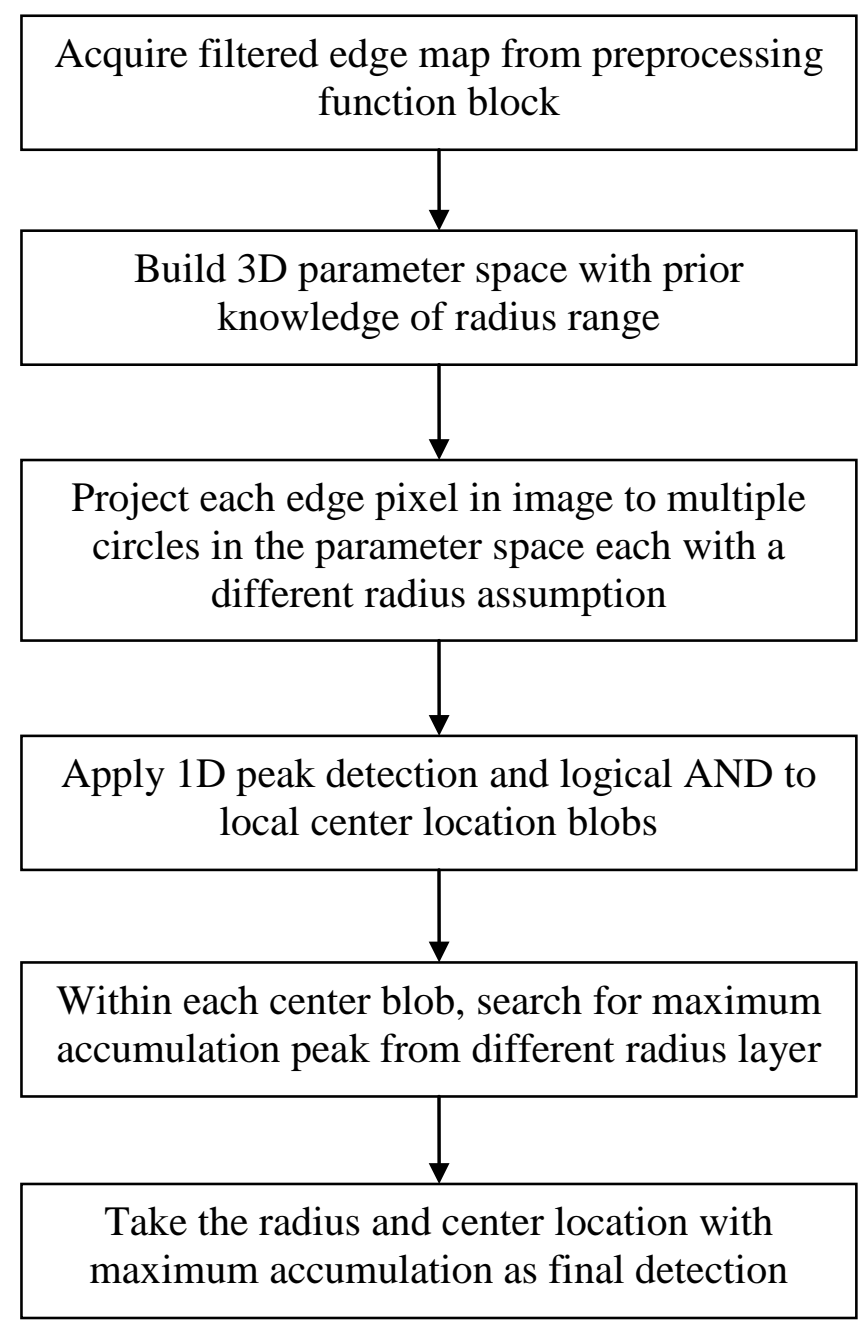

Figure 3.8 Function block diagram of regular Hough Transform realization

According to standard circle function, a circle in the image space is projected to many circles in the parameter space, and all intersected at real circles center coordinate. If take the parameter space as an accumulation space counting passing times of parameter circles, the shape feature detection is then converted to a peak finding problem.

The base of the 3D accumulation space is the same size as input edge map, and its height is equal to the range of radius variation, which is 25 to 40 in this case, each layer 
represents a different radius value. When projecting an edge pixel to one layer of the parameter space, the corresponding circle's circumference is calculated with one degree step size and rounded to integers to fit coordinate system. It also meets the resolution requirement in this study. However, if in a higher resolution condition, the degree resolution of theta should be increased to maintain pixel level connection of circumference as well.

(3.6) to (3.8) show how a circumference point $\left(x_{0}, y_{0}\right)$ is derived from radius and angle theta.

$$
\begin{aligned}
& x_{0}=\operatorname{round}(\text { radius } \cdot \cos (\theta)) \\
& y_{0}=\operatorname{round}(\text { radius } \cdot \sin (\theta)) \\
& \theta=0 \sim 360 \text { degree }
\end{aligned}
$$

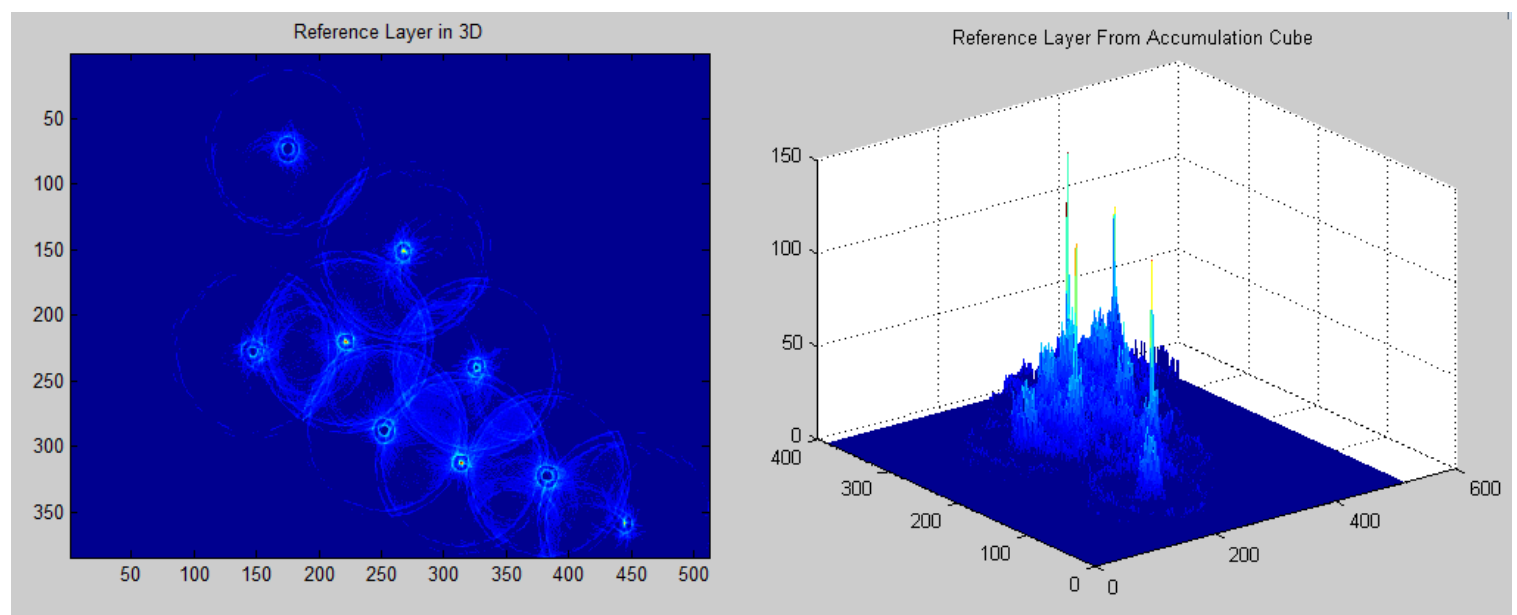

Figure 3.9 Filtered reference layer of Hough Transform parameter space

A reference layer is selected as the layer with global maximum accumulation peak, based on the assumption that most beads in the same image have same size of appearance, and more likely to concentrate high peaks. 
The purpose of taking out a reference layer is to locate rough center blobs which considered being the rough location estimation of circle centers. It is a way to breaking down a 3D peak searching problem into three 1D peak searching procedures. At first, 1D peak finding function is applied along each column and each row of this reference layer, with parameter setting such as minimum peak threshold and near peak distance threshold, as shown in Figure 3.10. The red and yellow cross indicated the peaks' locations along column and row direction.
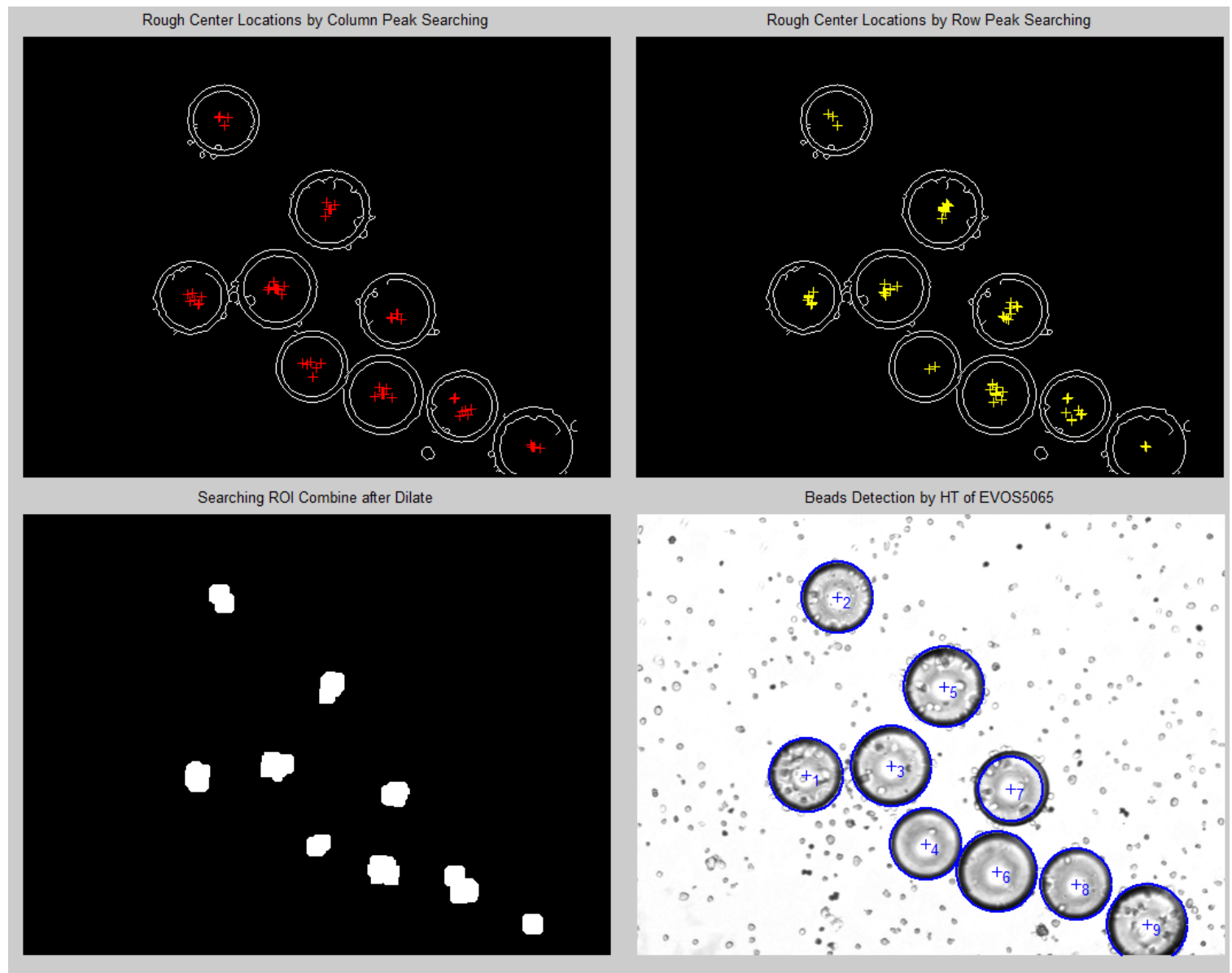

Figure 3.10 Peak searching and final detection of regular Hough Transform

Since circle is a uniform shape and symmetric along any orientation, the 1D peak finding procedure would return relative similar results from both directions. Afterwards, 
an image dilation kernel is applied to these peak locations, followed by a logical AND operation to group the two peak searching results together to form some blob area for another 1D maximum value searching along the radius axis of this parameter space.

The final step is to separately find the maximum value within each of those peak blobs along radius axis from different parameter layers, the layer of the maximum value corresponds to the best fitted radius, and maximum location of that frame represents the true circle center location.

Final detection results are satisfying with regular structure of Hough Transform. However, regular Hough Transform suffers slow processing speed and large memory requirement. The reason is the huge data amount in the 3D parameter space, just imagine that one single pixel in the edge map could become 360 pixels in just one layer of the

parameter space. These parameter space data significantly increases the computation load for building parameter space and 3D peak searching.

\subsection{Modified Hough Transform with gradient information}

From the previous algorithm developments, it can be noticed that geometric based method has less data in only one searching plane called energy map, and thus it processes faster. But its expanding circumference method for radius searching is not accurate when circles are incomplete or deformed. On the other hand, in Hough Transform parameter space, since only circles with correct radius could intersect at center, detected peak always corresponding to true radius value. But it is slow due to the huge amount of information that needs to be processed in the parameter space.

The idea of this algorithm is to integrate gradient orientation to regular Hough Transform to combine the advantages of them. So for each layer of the parameter space, 
instead of accumulating circumference points of parameter circles, only two points on both sides of an edge pixel, along its gradient direction and with the same radius distance, are placed for accumulation.

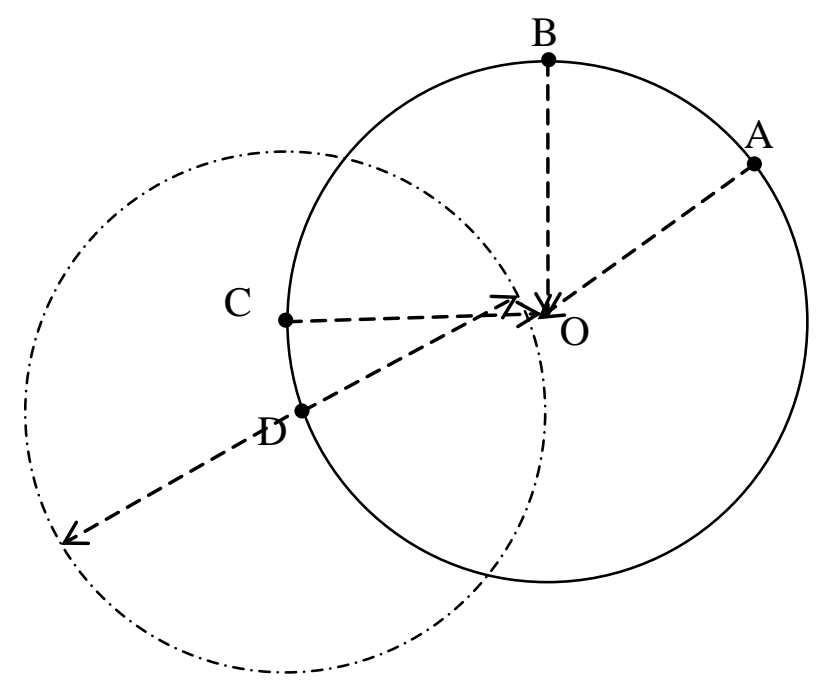

Figure 3.11 Accumulation with radius and gradient orientation in a parameter layer

Illustrated in Figure 3.11, edge pixel A, B and C effectively contribute to the peak accumulation at true circle center $\mathrm{O}$ with not only correct gradient angle, but also correct radius length. The point $\mathrm{D}$ with wrong gradient orientation points to somewhere else and added nothing to center point $\mathrm{O}$. The advantage with scattering points with wrong gradients is that there would not be fault peak accumulation comparing to draw lines. If lines cross whole frame are drawn as in the geometric based method, they could have accumulated peaks at wrong position as well.

It is chosen to place points on both sides of gradient orientation, pointing to and away from center, like point $\mathrm{D}$. The reason is that in the dataset many images have many beads and they connect to each other. An extra point put against gradient orientation may 
possibly contribute to a nearby circle's center and increased that voting. Because when data points in parameter space are reduced from a straight line to only a point of each edge pixel, every point becomes very influential. If there is no bead connected, the extra point would just be part of the background. Experiments show that this modification increases the detection rate.

The new parameter space shares the same 3D structure as regular Hough Transform. But the each parameter layer is in fact an energy map with gradient and radius variable derived from edge detection. The overall program flowchart is almost the same as regular Hough Transform, but because of data reduction, the processing time become 50-100 times faster than regular Hough Transform when run in MATLAB.

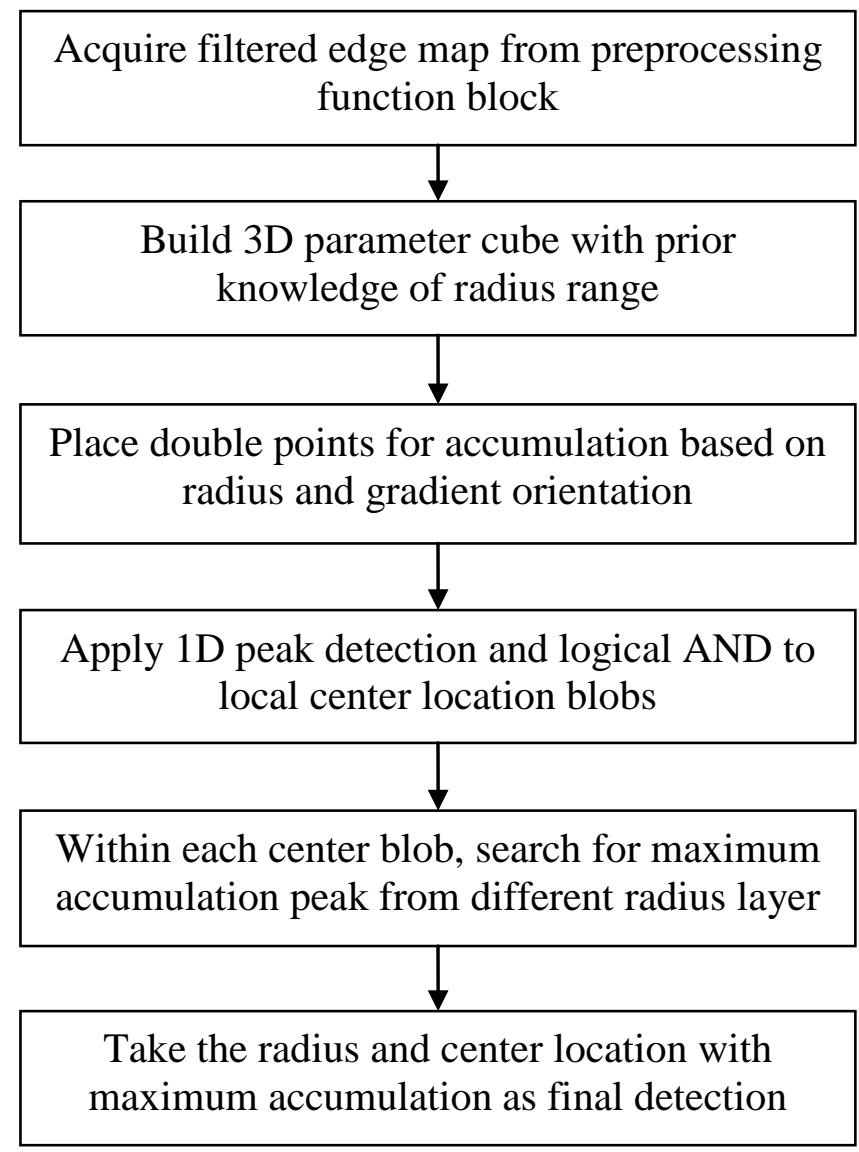

Figure 3.12 Function block diagram of Modified Hough Transform 


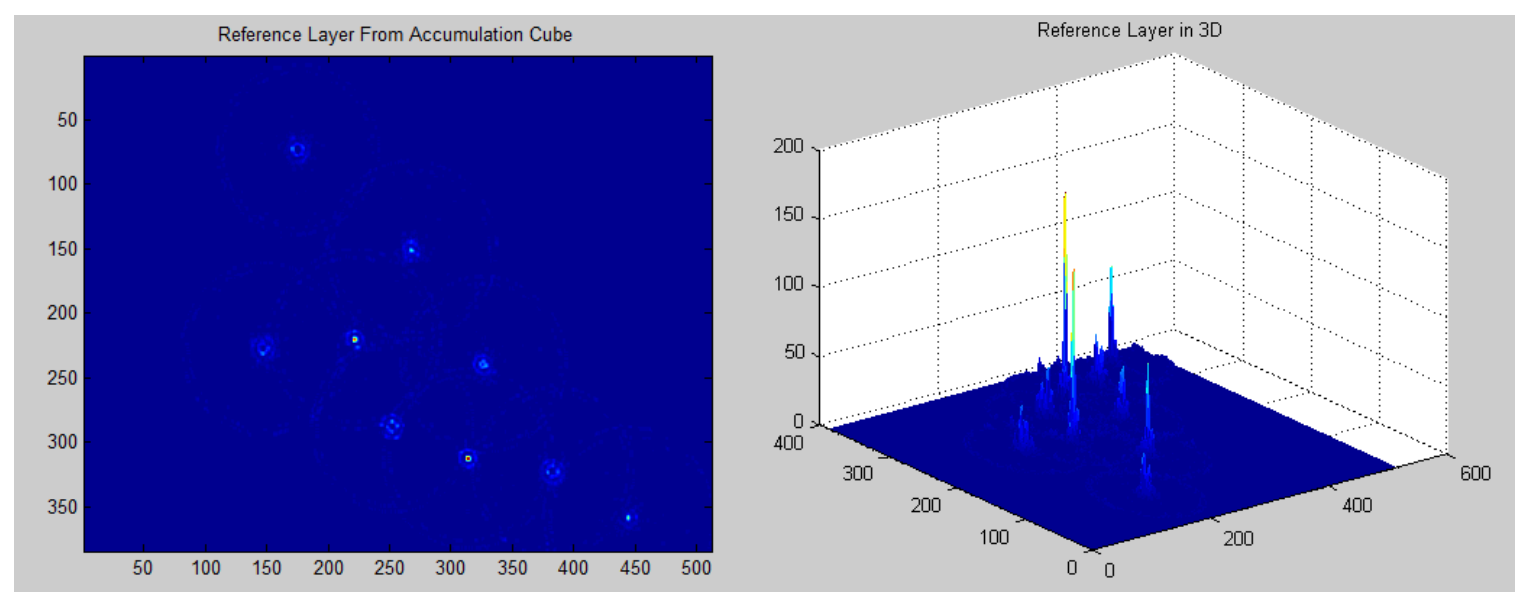

Figure 3.13 Reference layer of Modified Hough Transform parameter space

It is easy to discriminate the remarkable difference of parameter data amount when comparing Figure 3.13 and Figure 3.9 of regular Hough Transform. The values in the parameter layers of modified Hough Transform are amplified by an empirical factor of 10 to enhance the parameter setting tolerance of peak searching function. Final detection result is as good as regular Hough Transform. Because the clear parameter space increases the margin of adjusting peak searching parameters, the same setting achieves a high detection rate over all images than regular Hough Transform. 


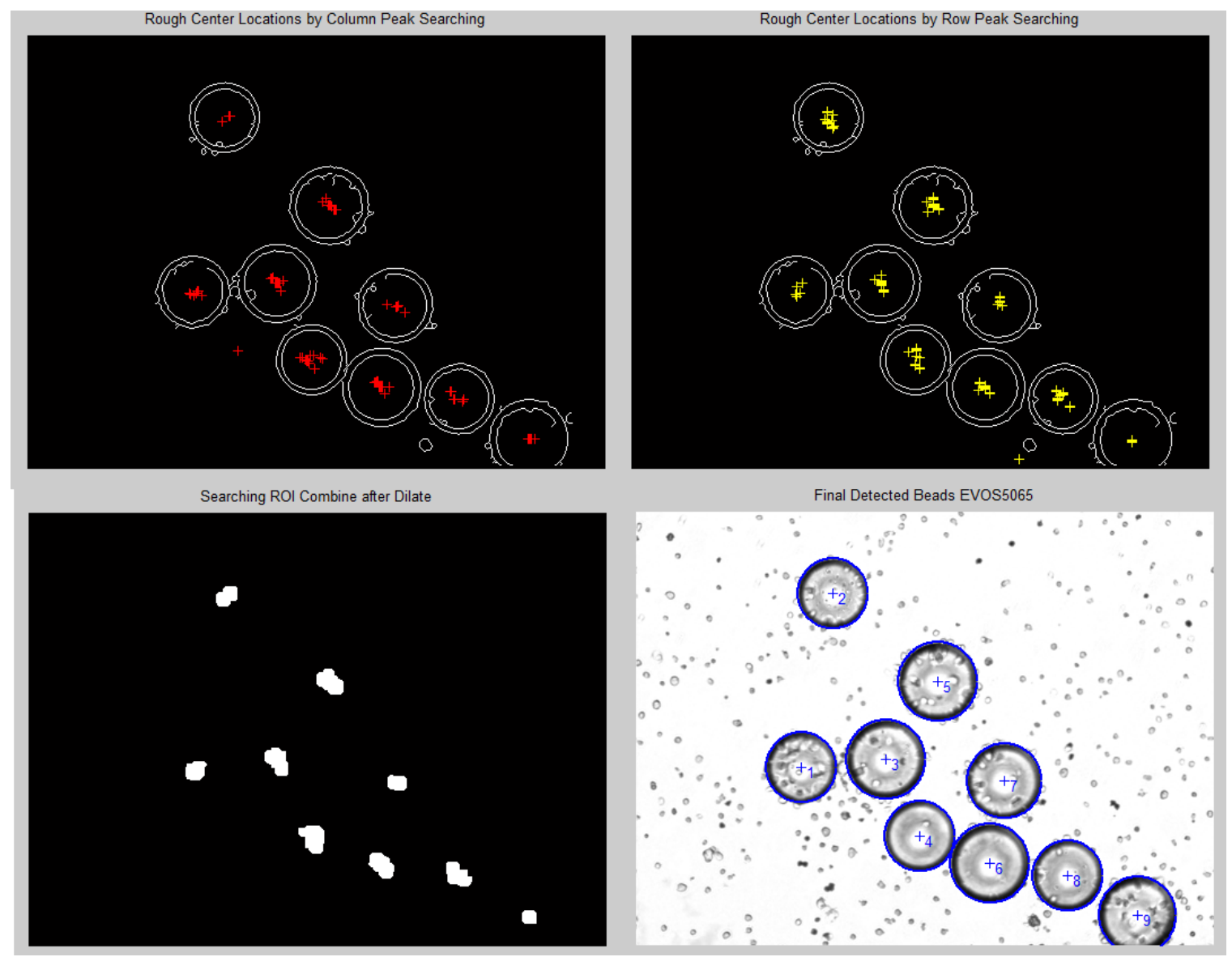

Figure 3.14 Peak searching and final detection of modified Hough Transform

\subsection{Real valued Artificial Immune System}

As an exploration of the power of Artificial Immune System, a real valued Clonal Selection Algorithm based multi-circle detection program is s designed to effectively detect beads in this chapter.

There are two types of B cell agents in this real valued Artificial Immune System, plasma cell and memory cell as discussed before in 2.2.2 of Clonal Selection Algorithm model. In this implementation of Clonal Selection, the antigens are circular edges from 
preprocessing step, antibodies are 3 random points on the edges, and memory cells are detected center coordinates and radii information.

The real valued representation is based on the chain indexing of edge segments. All edge segments are nonzero pixels in the black edge map and can be grouped and labeled according to 8-connectivity rule. For a random edge pixel $(x, y)$, any nonzero pixel in its surrounding neighborhood according to Figure 3.15 is considered to be connected with $(x, y)$ either horizontally or vertically, or diagonally.

\begin{tabular}{|l|l|l|}
\hline$(x-1, y-1)$ & $(x-1, y)$ & $(x-1, y+1)$ \\
\hline$(x, y-1)$ & $(x, y)$ & $(x, y+1)$ \\
\hline$(x+1, y-1)$ & $(x+1, y)$ & $(x+1, y+1)$ \\
\hline
\end{tabular}

Figure 3.15 Neighborhood of 8-connectivity rule

The chain indexing of labeled edge segments is achieved by traversing each of the edge segments.

1) The chain indexing pointer starts from the segment point which has the smallest coordinates, and uses 8-connectivity rule to clockwise trace the whole edge segment.

2) If there are branches on the main edge, the tracing pointer will visit every edge branch and return to the branch node to continue to next branch, until the end point.

3) If the edge segment is an enclosed loop, the end point will be the exact same starting point of traversing. If the edge is an open loop, the traversing pointer has to go from the graphical end point all the way back to starting point via coming path.

4) The chain indexing is the order of every edge pixel being visited during this traversal process stored in a 1D array. Because edge branches and open edge segments may be 
visited by tracing pointer twice on returning, the final indexed edge segment array is usually longer than the real edge segment.

This chain indexing traversal is very similar to the tree traversal algorithm in computer science. To this moment, each labeled and chain indexed edge segment becomes an antigen in this artificial immune system model.

The antibody in this model utilizes 3-point representation for circles. The 3-point representation is initially proposed in [59] for a Genetic Algorithm based shape detection method, and has been applied for circular shape detection in [74][77]. The concept is that an unknown circle has 3 parameters - two for the center coordinates $(a, b)$, and one for the radius $r$, then only 3 non-collinear points $\left(x_{1}, y_{1}\right),\left(x_{2}, y_{2}\right)$ and $\left(x_{3}, y_{3}\right)$ from circumference are good enough to solve the following equation set and confirm corresponding circle location. More specifically, these three points have the same integer index interval $\Delta$.

$$
\begin{aligned}
& \text { A real valued antibody: } \begin{array}{|l|l|l|}
\hline \text { Index } 1, x_{1}, y_{1} & \text { Index } 2, x_{2}, y_{2} & \text { Index } 3, x_{3}, y_{3} \\
\text { Index } 2=\text { Index } 1+\Delta, \text { Index } 3=\text { Index } 1+2 \cdot \Delta
\end{array}
\end{aligned}
$$

Figure 3.16 Real valued antibody encoding

A circle is determined by the real valued antibody according to (3.9).

$$
\left\{\begin{array}{l}
\left(x_{1}-a\right)^{2}+\left(y_{1}-b\right)^{2}=r^{2} \\
\left(x_{2}-a\right)^{2}+\left(y_{2}-b\right)^{2}=r^{2} \\
\left(x_{3}-a\right)^{2}+\left(y_{3}-b\right)^{2}=r^{2}
\end{array}\right.
$$

Then $a, b$ and $r$ can be solved from (3.9) using (3.10) - (3.12),

$$
a=\frac{\left|\begin{array}{ll}
x_{2}^{2}+y_{2}^{2}-\left(x_{1}^{2}+y_{1}^{2}\right) & 2\left(y_{2}-y_{1}\right) \\
x_{3}^{2}+y_{3}^{2}-\left(x_{1}^{2}+y_{1}^{2}\right) & 2\left(y_{3}-y_{1}\right)
\end{array}\right|}{4\left[\left(x_{2}-x_{1}\right)\left(y_{3}-y_{1}\right)-\left(x_{3}-x_{1}\right)\left(y_{2}-y_{1}\right)\right]}
$$




$$
\begin{aligned}
& b=\frac{\left|\begin{array}{ll}
2\left(x_{2}-x_{1}\right) & x_{2}^{2}+y_{2}^{2}-\left(x_{1}^{2}+y_{1}^{2}\right) \\
2\left(x_{3}-x_{1}\right) & x_{3}^{2}+y_{3}^{2}-\left(x_{1}^{2}+y_{1}^{2}\right)
\end{array}\right|}{4\left[\left(x_{2}-x_{1}\right)\left(y_{3}-y_{1}\right)-\left(x_{3}-x_{1}\right)\left(y_{2}-y_{1}\right)\right]} \\
& r=\sqrt{\left(x_{1}-a\right)^{2}+\left(y_{1}-b\right)^{2}}
\end{aligned}
$$

In the antibody, Index1, Index 2 and Index3 with the same index interval are the connection between antigen and antibody.

The affinity ratio in this model is a fitness measurement to evaluate the effectiveness of an antibody to antigens. Assume there is an indexed edge segment as antigen and an antibody stimulates from this antigen. An array antigen $\mathbf{P}$ with same length as edge segment is initialized with value 1 , and for the antibody initializes another same size array $\mathbf{Q}$. If the circumference derived from antibody falls on the same location of existing edge point, set Q's value to 1, otherwise set it 0 .

\begin{tabular}{l|llllllll}
\hline Antigen P: & 1 & 1 & 1 & 1 & $\ldots \ldots$ & 1 & 1 & $\ldots \ldots$ \\
\hline Antibody Q: & 0 & 1 & 1 & 0 & $\ldots \ldots$ & 0 & 0 & $\ldots \ldots$ \\
\hline Edge segment point: & $\mathrm{pt}_{1}$ & $\mathrm{pt}_{2}$ & $\mathrm{pt}_{3}$ & $\mathrm{pt}_{4}$ & $\ldots \ldots$ & $\mathrm{pt}_{\mathrm{n}-1}$ & $\mathrm{pt}_{\mathrm{n}}$ & $\ldots \ldots$ \\
\hline
\end{tabular}

Figure 3.17 Affinity representation

The affinity is calculated in the similar way as Hamming distance between $\mathbf{P}$ and Q. The affinity is in fact calculated by checking how many edge pixels really are located on the derived antibody circumference.

The real valued clonal selection algorithm performs on the same preprocessed edge image as the three previous programs. The antigens are the white edges on the black background, and B cell antibody would be a 3-point representation of a candidate circle. Antibodies with affinity above a threshold will be considered effective to destroy 
antigens, and detected circle's center and radius information is transformed to memory cells for later results output. The algorithm performs in the order of these steps,

1) All continuous edges are labeled and chain indexed using 8-connectivity rule. Each labeled edge segment is treated as an antibody and a 3-point combination representation of B cell antibody would be stimulated from the edge segment.

2) Randomly select a point from the chain index array and two other points with interval $\Delta$. The indexes and coordinates of the three points are encoded as antibody in the form of a real valued vector as Figure 3.16. The integer $\Delta$ varies according to the mechanism in Figure 3.19.

3) The possible longest circumference of one circle should be $2 \pi r_{\max }$. Since in this program $r$ is set in the ranges of 25 to 40, it is approximated by a round figure of 250 . The affinity ratio is then calculated for two conditions.

Affinity1, when an edge is shorter than 250 it is considered to be one circle or an arc, the affinity ratio is the sum of existing pixel on circumference over correspond edge segment’s length.

Affinity2, when circumference is longer than 250, it is considered as multiple circles connected, it is defined as existing pixel on edge circumference over derived single circumference length.

$$
\text { affinity ratio }=\left\{\begin{array}{l}
\frac{\operatorname{sum}(\mathbf{Q})}{\operatorname{sum}(\mathbf{P})}, \text { if edge length }<250 \\
\frac{\operatorname{sum}(\mathbf{Q})}{\text { length }(\mathbf{Q})}, \text { if edge length }>250
\end{array}\right.
$$

4) When the affinity ratio exceeds a threshold of $30 \%$, double check the corresponding radius. If it is within predefined range, it is considered to be an effective antibody, edge segments regarding detected circle are erased just like the antigen is destroyed 
by antibody. The erasing is done by a dilation operation along antibody's circumference and then set dilated area to 0. Finally, antibody center and radius information will be transferred to a memory cell and stored separately. Otherwise, abandon the antibody candidate. This will help to prevent keeping false little circle possibly caused by the edge piece of a cancer cell.

5) For the abandoned antibody, a mutation is triggered for continuing antigen searching. The mutation of antibody is composed of a reselection of the first point and a variable interval for 3-point representation

The reselection of the three edge point is completed by adding a random positive integer shift to the old first point index. The modular arithmetic is applied here and remainder is obtained to transform the linear addition to a random selection on a rotational sequence like roulette wheel, no matter the new Index1 is within the real array range or not.

$$
\begin{gathered}
\text { shift }=\operatorname{rand}\left(\frac{\text { edge_size }}{3}\right) \\
\text { Index } 1_{\text {new }}=\operatorname{Rem}(\text { Index } 1+\text { shift }) \\
\Delta=-\log _{1.1} \text { (affinity) }
\end{gathered}
$$

\begin{tabular}{ll|l|l|} 
Mutated antibody: & Index $1_{\text {new }}, x_{1}, y_{1}$ & Index $_{\text {new }}+\Delta, x_{2}, y_{2}$ & Index $_{\text {new }}+2 \cdot \Delta, x_{3}, y_{3}$ \\
\cline { 2 - 4 }
\end{tabular}

Figure 3.18 New mutated antibody

The interval $\Delta$ is controlled by the logarithm of affinity ratio to base value 1.1, which gives a longer interval size when affinity ratio is low. The base should be a little bit larger than 1 because affinity is always equal or less than 1 . The base value 
can be adjusted for different mutation sensitivity requirements. This mechanism will help the antigen jump out irregular part of an edge segment. An exponential function would provide the same effect.

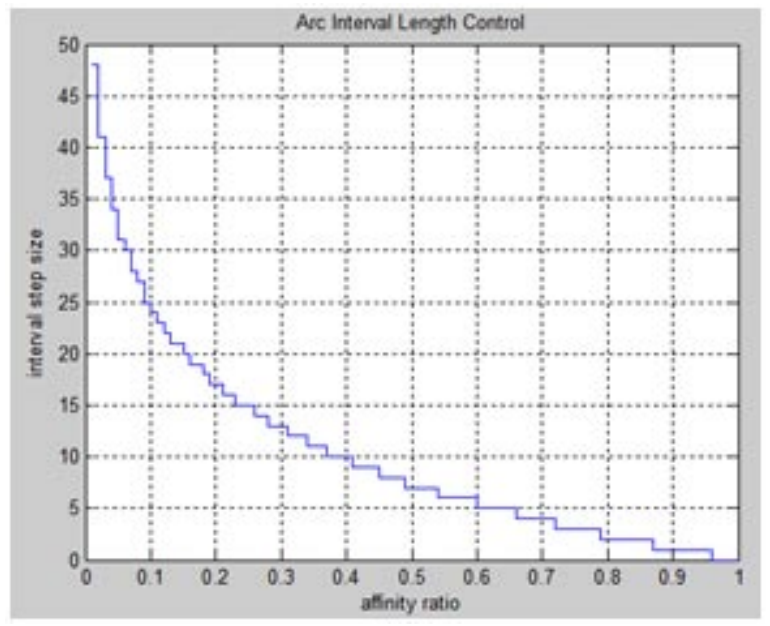

Figure 3.19 Control mechanism of $\Delta$ for real valued antibody

The $\Delta$ curve is obtained by discretization. In fact, any curve convex to origin would work for adjusting $\Delta$. Attention should be paid to the curvature and two end values.

6) Go back to step 2) and repeat through. Every time when updating memory cells also check the center distance between new and old circles, update old circles with new ones with higher affinity if the distance is very close.

7) Stop the iteration until all remaining edge segments are shorter than 50, or a total iteration time of 35 as the age limitation is reached. 


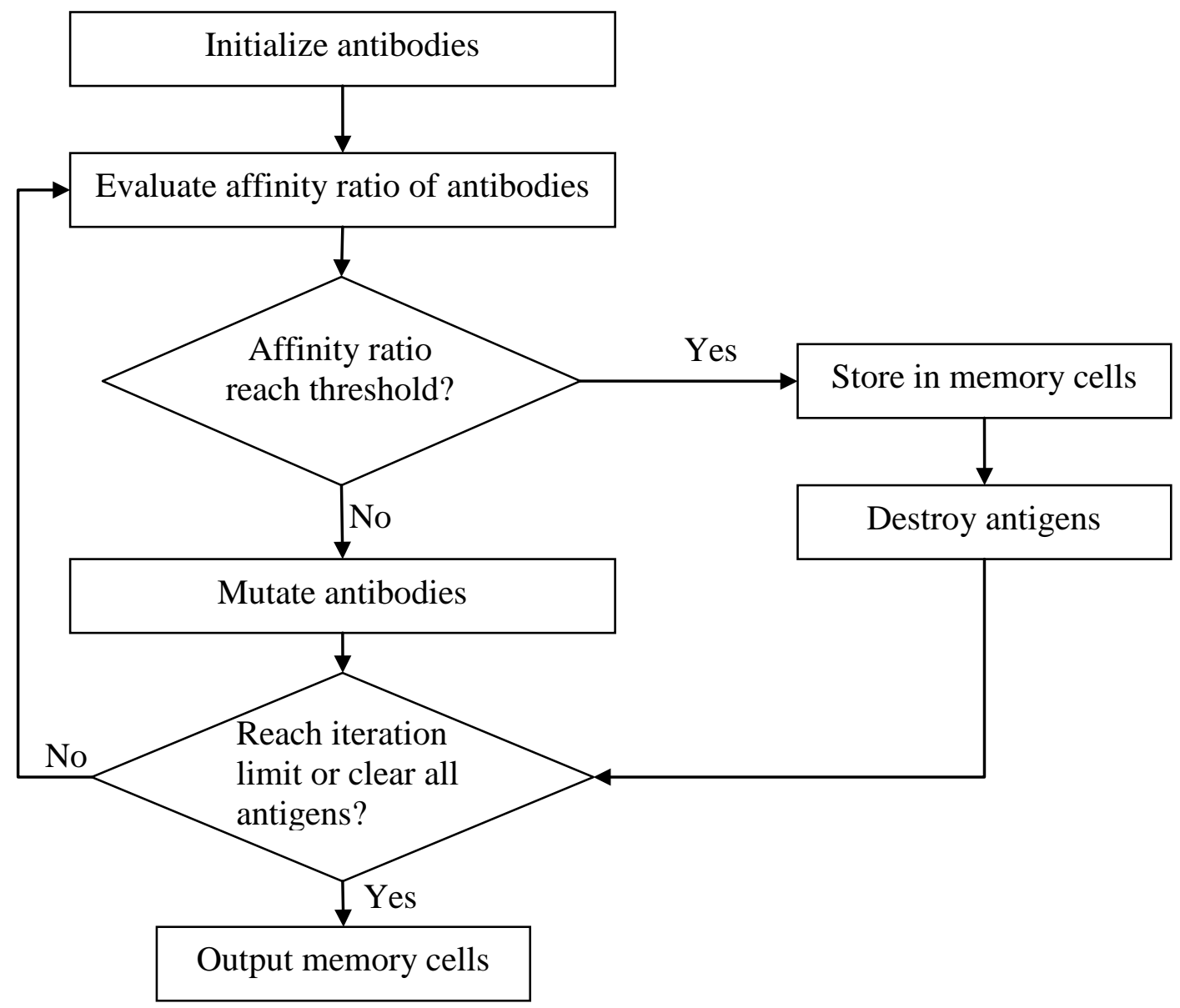

Figure 3.20 Function block diagram of Clonal Selection Algorithm

When Clonal Selection Algorithm is finished, output the detected circles by drawing circles according to the center and radius information stored in memory cells. Result is shown in Figure 3.22. Figure 3.21 shows the edge map before and after processing, where most white edges are removed due to an effect of successful detection and destruction. 


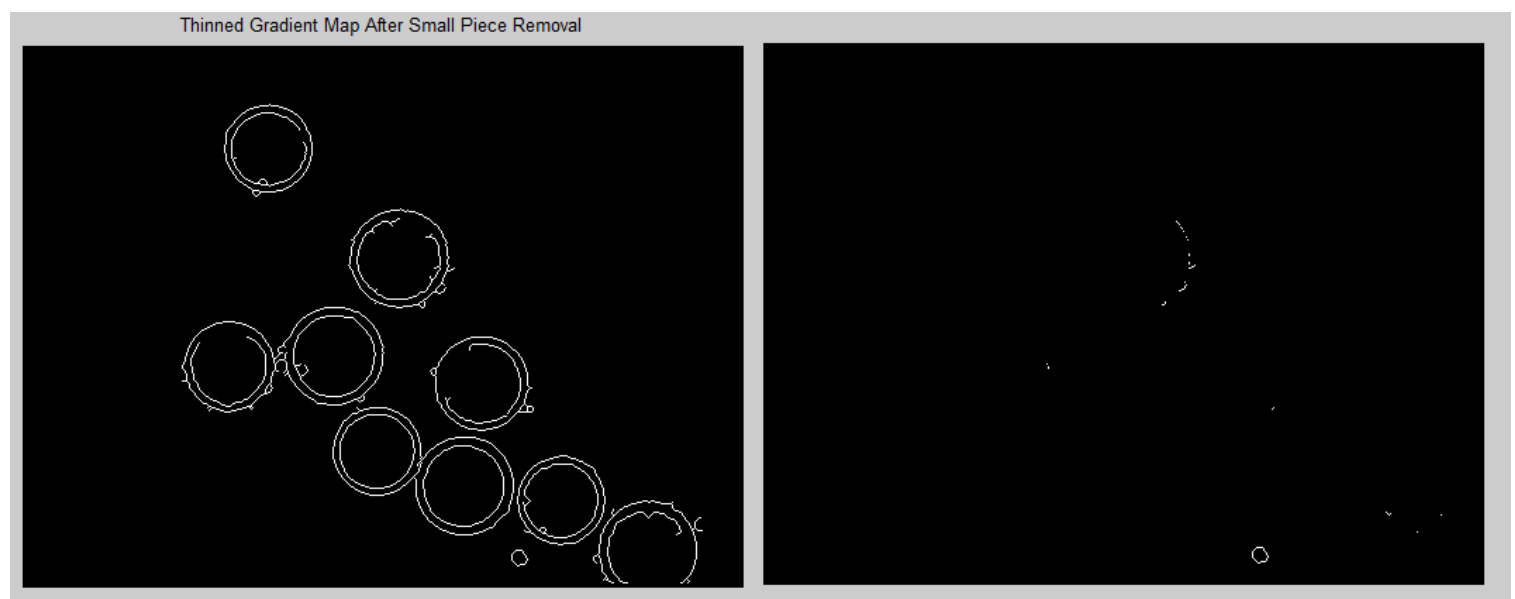

Figure 3.21 Edge map before and after Clonal Selection Algorithm

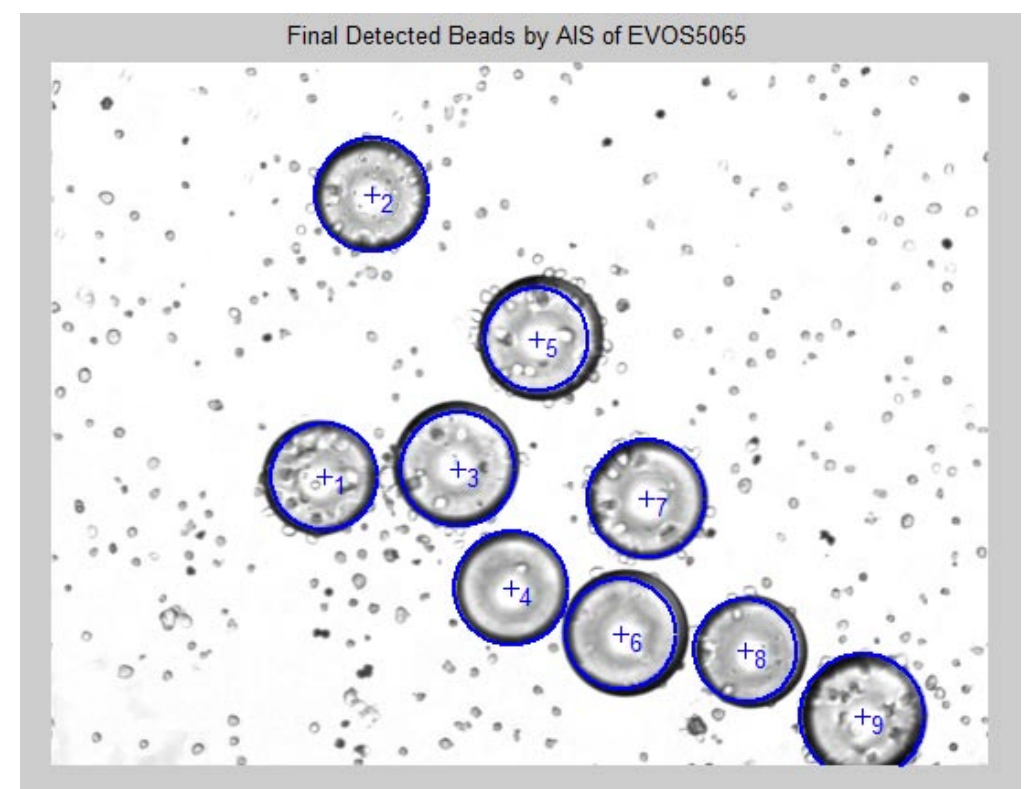

Figure 3.22 Circle Detection results with real valued Clonal Selection Algorithm

Since Cuevas already published an AIS Clonal Selection based circle detection algorithm [74], a comparison is made here to show the difference and effectiveness of this real valued approach. 
Table 3-1 Comparison between binary and real valued AIS

\begin{tabular}{|l|l|l|}
\hline & $\begin{array}{l}\text { Binary Clonal Selection based Multi- } \\
\text { circle Detection }\end{array}$ & $\begin{array}{l}\text { Real valued Clonal Selection based } \\
\text { Multi-circle Detection }\end{array}$ \\
\hline 1 & $\begin{array}{l}\text { Able to detect incomplete circular } \\
\text { segments, arcs }\end{array}$ & $\begin{array}{l}\text { Able to detect incomplete circular } \\
\text { segments, arcs }\end{array}$ \\
\hline 2 & Antibody is binary string & Antibody is real valued vector \\
\hline 3 & Fixed population size & $\begin{array}{l}\text { Population size depended on the number } \\
\text { of edge segments }\end{array}$ \\
\hline 4 & $\begin{array}{l}\text { Randomly generated 3-point } \\
\text { representation of candidate circles over } \\
\text { whole image and verify existence }\end{array}$ & $\begin{array}{l}\text { 3-point with equal chain index } \\
\text { distance randomly generated on } \\
\text { existing edge segments }\end{array}$ \\
\hline 5 & $\begin{array}{l}\text { Matching function minimizes affinity } \\
\text { index to zero }\end{array}$ & $\begin{array}{l}\text { Matching function increases affinity } \\
\text { ratio to one }\end{array}$ \\
\hline 6 & $\begin{array}{l}\text { Evaluate distinctiveness by center location } \\
\text { and radius size }\end{array}$ & $\begin{array}{l}\text { Evaluate distinctiveness by center } \\
\text { location and affinity ratio }\end{array}$ \\
\hline 7 & $\begin{array}{l}\text { Mutate by flipping bits of binary string } \\
\text { Mutate by randomly placing first point } \\
\text { location of 3-point set with varying } \\
\text { index intervals inversely proportional } \\
\text { to affinity ratio }\end{array}$ \\
\hline 8 & $\begin{array}{l}\text { Selection finishes, memory kept all Clonal } \\
\text { memory to find local maxima for other } \\
\text { circles }\end{array}$ & $\begin{array}{l}\text { Use memory cells to keep detected } \\
\text { circle and return all results once when } \\
\text { Clonal Selection finishes }\end{array}$ \\
\hline $\begin{array}{l}\text { Use 90 antigens to achieve 0.1 fitness, (the } \\
\text { matching function minimized fitness to 0, } \\
\text { so 0.1 is equivalent 90\% ) }\end{array}$ & $\begin{array}{l}\text { On average use 35 iteration to achieve } \\
\text { 90\% precision }\end{array}$ \\
\hline
\end{tabular}

The advantage of using real valued vector representation is that it guarantees all antigens would still stay on edge segments rather than being placed on black background during the clonal mutation process, while binary string representation with bits flipping mutation scheme is not controllable. The addition of memory cell storing valid detection saves more memory space than keeping record of the whole process for local maxima retrieval. 
Also because of the similarity between Genetic Algorithm and Clonal Selection Algorithm, a Genetic Algorithm based circle detection program is implemented as well to show the effect of a single detector taking over the population discussed in [70].

This Genetic Algorithm performs on the same edge map as previous methods and uses binary string representation of center and radius combination. The population size is 80 and stopping criterion is 200 iterations. Once randomly initialized population, a roulette wheel selection operation is used to pick out parents for 1-point crossover and individuals for 1 -point mutation. Cross over rate is set to $80 \%$ and mutation rate is $10 \%$ of entire population. Child chromosomes would replace those individuals with lowest fitness in that generation, while mutate chromosomes replace themselves for next generation. A fitness function evaluates the overlapping rate of existing edges and circle candidates from GA. The final output result is the chromosomes with fitness over 0.4.

In left image of Figure 3.23, after the evolution process, fitted Genetic Algorithm circle chromosomes all converge to one bead which is repeatedly highlighted by red color. Multi-circle detection could only be accomplished by introducing a huge memory space to store all processing iterations and going back for local maxima retrieval as suggested by [51][52][74]. 


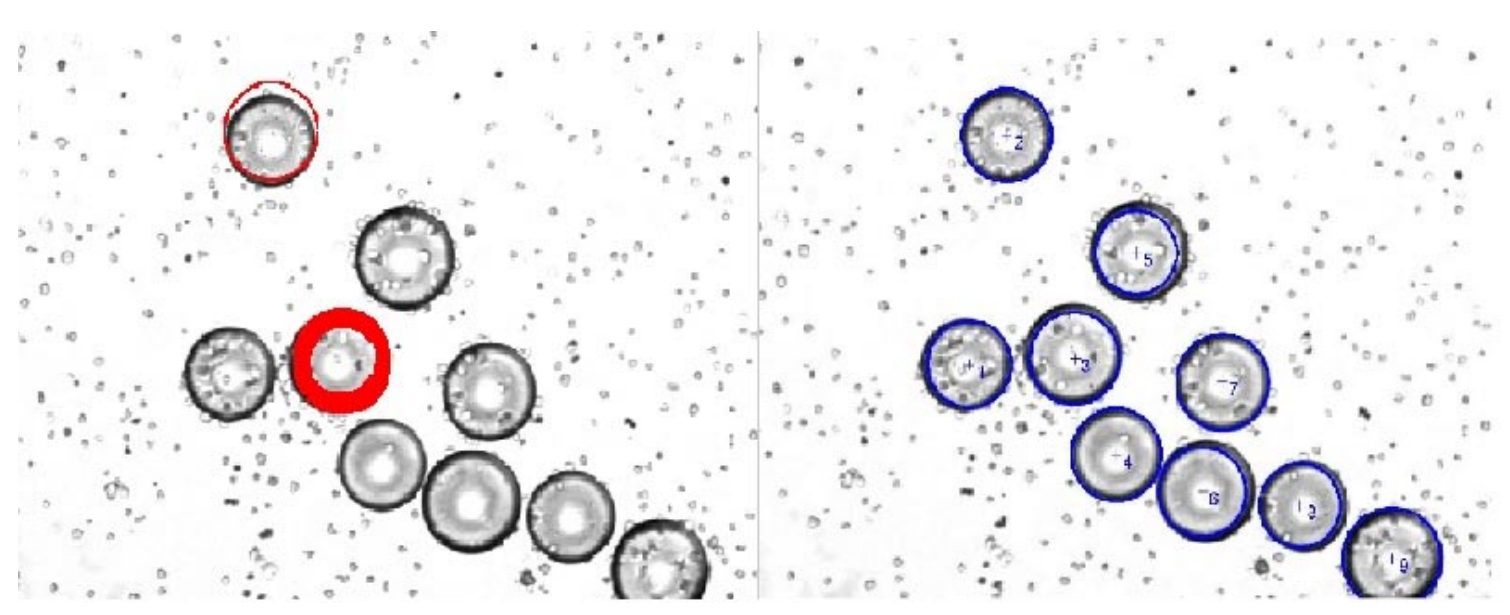

Figure 3.23 Comparison of GA (left) and AIS (right)

Table 3-2 Comparison between Genetic Algorithm and Artificial Immune System

\begin{tabular}{|l|l|l|}
\hline & Genetic Algorithm & $\begin{array}{l}\text { Artificial Immune System (Clonal } \\
\text { Selection) }\end{array}$ \\
\hline 1 & Population of chromosomes & $\begin{array}{l}\text { Population of antibodies and memory } \\
\text { cells }\end{array}$ \\
\hline 2 & Binary string of chromosome & Real valued vector of antibody \\
\hline 3 & Center, radius representation of circles & 3 non-collinear points representation \\
\hline 4 & Fixed population size & $\begin{array}{l}\text { Variable population size depended on } \\
\text { existing edge segments }\end{array}$ \\
\hline 5 & Fitness index based evaluation & Affinity based evaluation \\
\hline 6 & $\begin{array}{l}\text { Roulette wheel selection, } \\
\text { binary bits switching for crossover, } \\
\text { and bits flipping for mutation }\end{array}$ & $\begin{array}{l}\text { Real valued mutation, } \\
\text { different memory cell representation of } \\
\text { center and radius }\end{array}$ \\
\hline 7 & $\begin{array}{l}\text { Multiple run needed for multi-circle } \\
\text { detection }\end{array}$ & Run once for multi-circle detection \\
\hline
\end{tabular}




\section{CHAPTER 4 SIMULATION RESULTS}

There are 85 manually graded images in the dataset. They are used as raw dataset and standard as well for evaluation of the performance of four algorithms developed in this study. All algorithms are programmed in MATLAB 2009a. The performance of the algorithms is evaluated by the strategy called precision and sensitivity.

Precision and sensitivity are used in pattern recognition to evaluate classifier performance. Precision is defined as the fraction of retrieved instances that are relevant, while sensitivity is the fraction of relevant instances that are retrieved [78]. Taking a binary decision problem for example, one classier detects targets as either positive or negative. The decision made by this classier can be represented in a structure known as a confusion matrix. The confusion matrix has four categories:

1) True Positives are objects correctly labeled as positives;

2) False Positives are negative examples incorrectly labeled as positive;

3) True Negatives are negatives correctly labeled as negative;

4) False Negatives are positive examples incorrectly labeled as negative [79].

Table 4-1 Confusion matrix of precision and sensitivity

\begin{tabular}{|l|l|l|}
\hline & Actual Positive & Actual Negative \\
\hline $\begin{array}{l}\text { Detected } \\
\text { Positive }\end{array}$ & $\begin{array}{l}\text { True Positive } \\
\text { (correctly detected existing } \\
\text { circle) }\end{array}$ & $\begin{array}{l}\text { False Positive } \\
\text { (wrongly detected non- } \\
\text { existing circle) }\end{array}$ \\
\hline $\begin{array}{l}\text { Detected } \\
\text { Negative }\end{array}$ & $\begin{array}{l}\text { False Negative } \\
\text { (missing detection of existing } \\
\text { circle) }\end{array}$ & $\begin{array}{l}\text { True Negative } \\
\text { (N/A) }\end{array}$ \\
\hline
\end{tabular}


In the circle detection task, there is no applicable definition for True Negative, because the targeting process has only 3 situations as hit, miss and hit wrongly. Thus in this case, the precision and sensitivity definitions are modified as below.

$$
\begin{aligned}
& \text { precision }=\frac{\text { correct detection }}{\text { correct detection }+ \text { false detection }} \\
& \text { sensitivity }=\frac{\text { correct detection }}{\text { correct detection }+ \text { missing detection }}
\end{aligned}
$$

According to the definition of precision and sensitivity, the ideal circle detection method should correctly find all circles in image without missing or false detection. The precision and sensitivity would be both $100 \%$ in the ideal condition. In real condition, when correct detection is defined as finding both center and radius correctly, precision and sensitivity percentage will be expected to decrease.

All 85 images from dataset are processed by four algorithms with each of their own settings unchanged, which mean all the parameters are fixed when processing all images and no adjustments are made. This could help to compare the robustness of the algorithms under different background and objects conditions. Then processed results are compared with manually graded standards by person, and statistics are calculated. Graphs of precision and sensitivity evaluation are from Figure 4.1to Figure 4.3. 
Table 4-2 Statistics of precision for four algorithms

\begin{tabular}{|l|l|l|l|l|l|l|l|}
\hline & \multicolumn{4}{|l|}{ Total bead detection rate } & \multicolumn{3}{l|}{ Total bead detection number } \\
\hline Method & Max & Min & Mean & $\begin{array}{l}\text { Standard } \\
\text { deviation }\end{array}$ & $\begin{array}{l}\text { Correct } \\
\text { detection }\end{array}$ & $\begin{array}{l}\text { Incorrect } \\
\text { detection }\end{array}$ & $\begin{array}{l}\text { Missing } \\
\text { detection }\end{array}$ \\
\hline $\begin{array}{l}\text { Geometric } \\
\text { based }\end{array}$ & 1.00 & 0.00 & 0.89 & 0.193 & 1039 & 116 & 159 \\
\hline $\begin{array}{l}\text { Regular } \\
\text { HT }\end{array}$ & 1.00 & 0.25 & 0.94 & 0.129 & 1090 & 82 & 103 \\
\hline $\begin{array}{l}\text { Modified } \\
\text { HT }\end{array}$ & 1.00 & 0.88 & 0.99 & 0.025 & 1178 & 14 & 37 \\
\hline AIS & 1.00 & 0.79 & 0.97 & 0.039 & 1459 & 62 & 32 \\
\hline
\end{tabular}

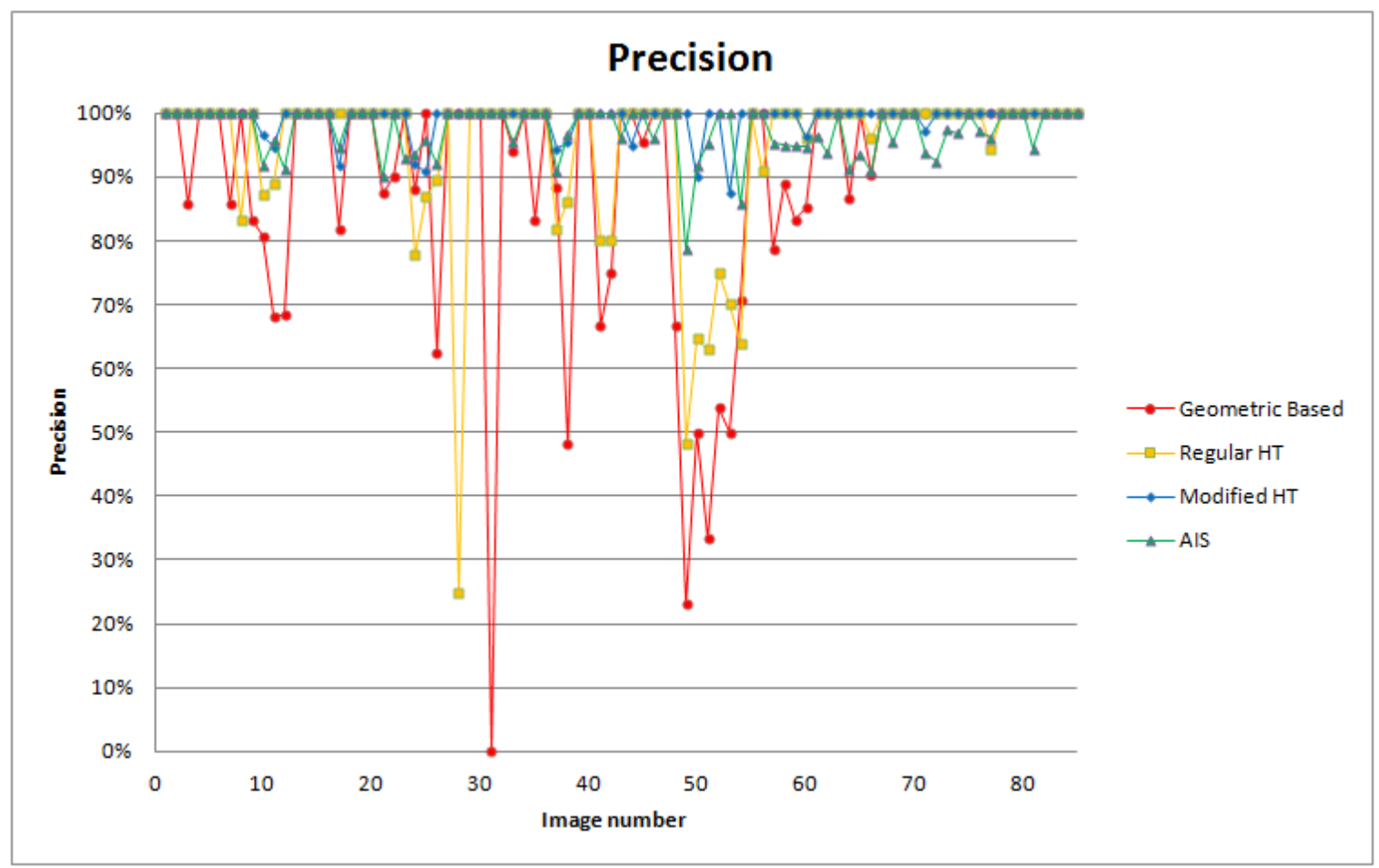

Figure 4.1 Precision graph of four algorithms

Precision is a measurement of the rate of correct detection over all detected circles. Perfect situation is a straight line all along $100 \%$ of y-axis in Figure 4.1 for all images. It is apparent that geometric based method has the worst results, which is not beyond expectation. When cancer cells attached to the surface of beads, the contour of 
beads acquired by edge detection are most likely not perfect circular shapes, and could not provide accurate gradient orientation information. Extreme case is a $0 \%$ meaning no detection at all. Regular Hough Transform also suffers a number of false detections with some images. The modified Hough Transform and Artificial Immune System are generally achieving correct detection above $90 \%$ with small standard deviation, which are superior to normal geometric feature based and Hough Transform.

Table 4-3 Statistics of sensitivity for four algorithms

\begin{tabular}{|l|l|l|l|l|}
\hline Method & Max & Min & Mean & Standard deviation \\
\hline Geometric based & 1.00 & 0.00 & 0.87 & 0.198 \\
\hline Regular HT & 1.00 & 0.56 & 0.93 & 0.104 \\
\hline Modified HT & 1.00 & 0.50 & 0.97 & 0.075 \\
\hline AIS & 1.00 & 0.73 & 0.98 & 0.049 \\
\hline
\end{tabular}

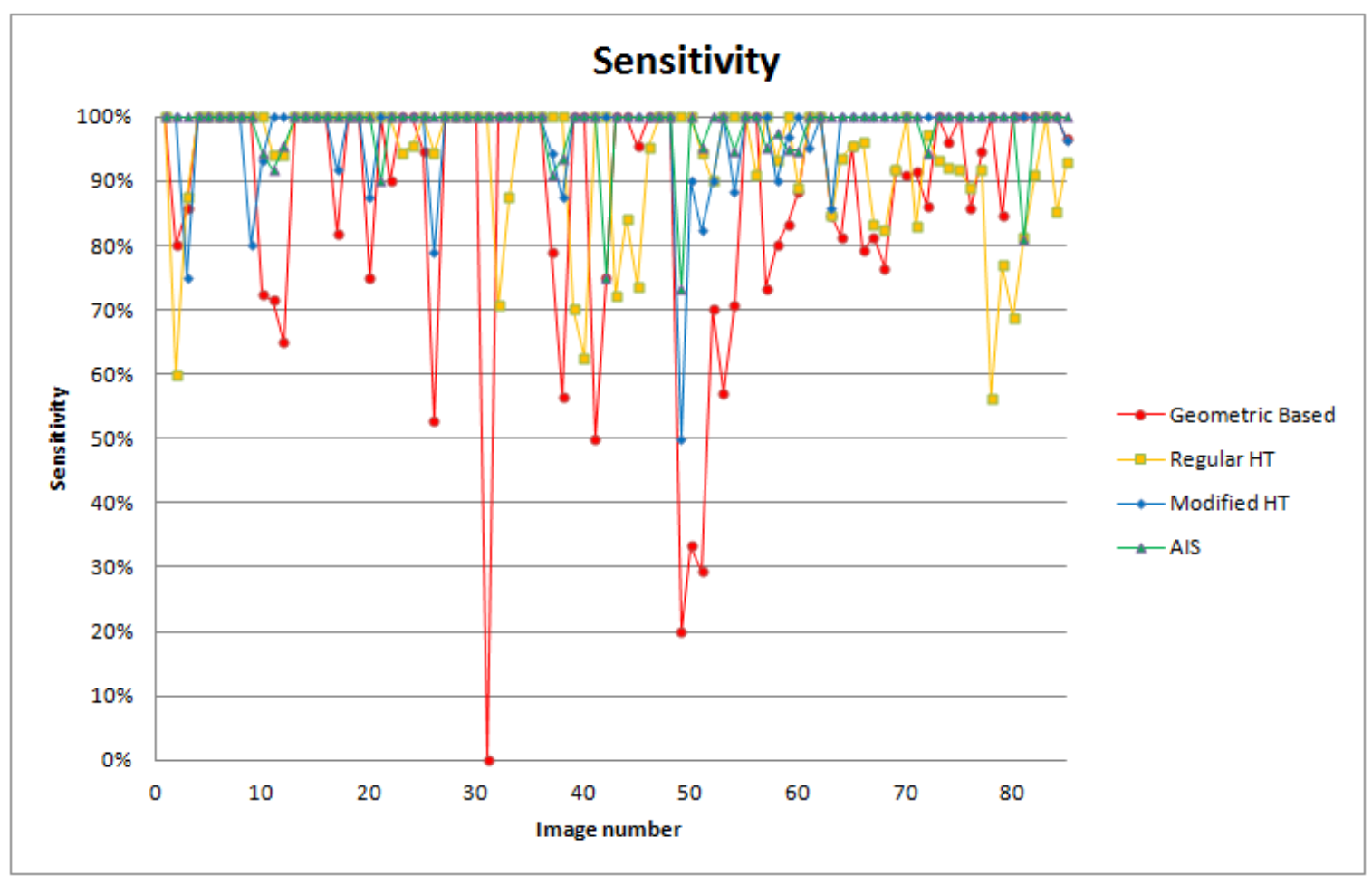

Figure 4.2 Sensitivity graph of four algorithms 
Sensitivity evaluates the detection rate over all existing circular shapes. Among all four algorithms, geometric based method, regular Hough Transform and its modified version are all deterministic approach and relied on several threshold settings. On one hand, a low sensitive setting would result in good precision numbers because detected circles must be true and distinctive, while sensitivity rate would be low because nonperfect shapes are missed. On the other hand, high sensitivity would improve sensitivity by reducing missing incidence but increase false detection rate at the same time.

The real valued Artificial Immune System model initializes antibodies on all edge segments, and resulted in an almost perfect $100 \%$ overall sensitivity, due to every edge segments are checked by antibodies during processing. In addition, with the inherent randomness and affinity orientated mutation, this stochastic method converges very fast as well.

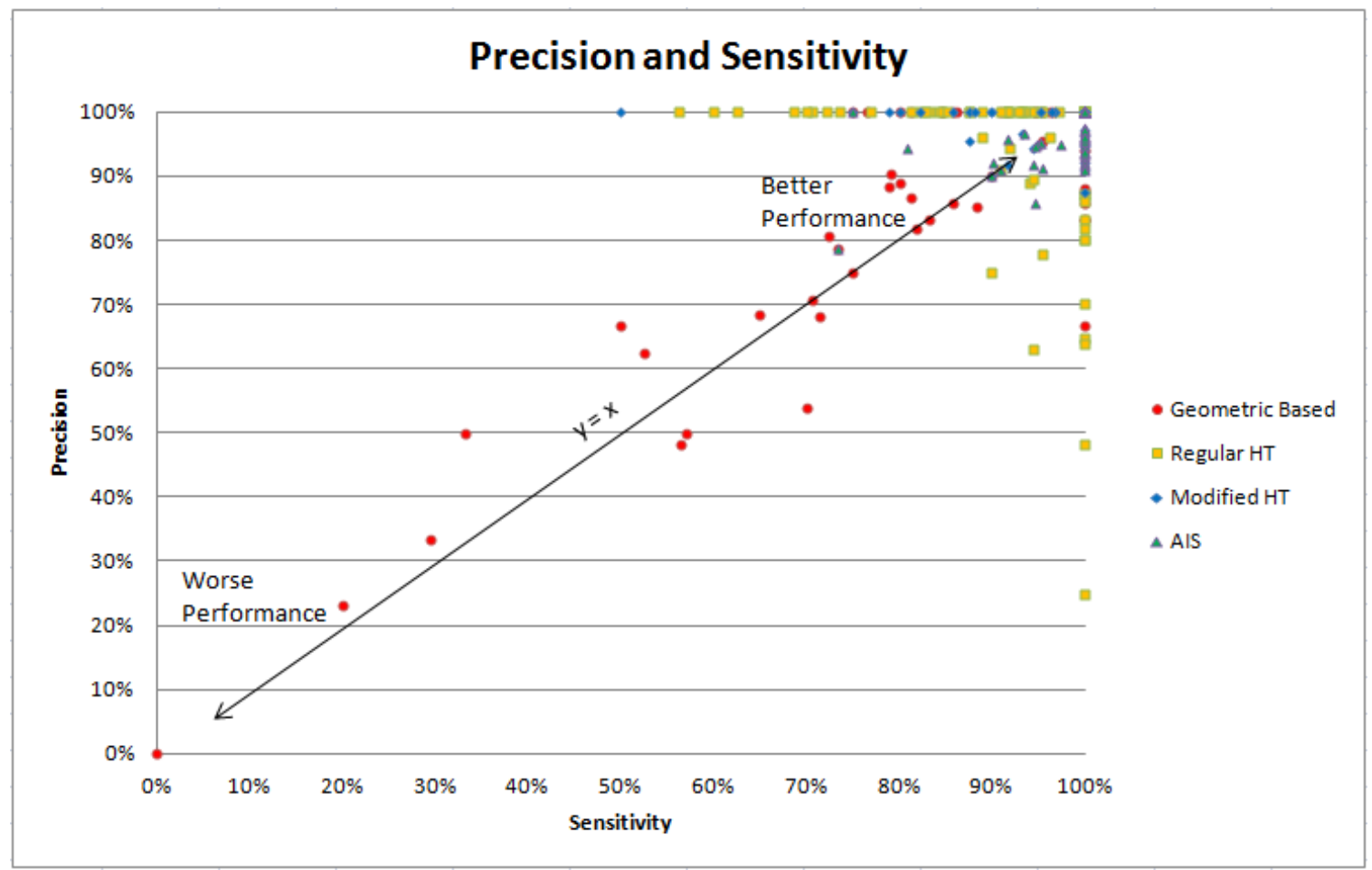

Figure 4.3 Precision and sensitivity graph of four algorithms 
If consider precision and sensitivity at the same time, the ideal result would be all data points centered at top right corner. The line of $y=x$ indicated a $50 \%$ equal probability detection rate, like blindly guessing yes or no. The scattered data samples show the advantage of real valued Artificial Immune System model over the other deterministic methods.

Some detection samples are illustrated from Figure 4.4 to Figure 4.7 to show the performance of these methods under different situations. In order to test the pixel level accuracy, these samples including the example in previous chapters are compared to manually obtained ground-truth pictures in Adobe Photoshop CS3. Detected beads' parameter $(a, b, r)$ are compared to their ground-truth circles ( $\left.a_{\text {true }}, b_{\text {true }}, r_{\text {true }}\right)$ using an error score function borrowed from [74].

$$
E s=\eta \cdot\left(\left|a_{\text {ture }}-a\right|+\left|b_{\text {true }}-b\right|\right)+\mu \cdot\left|r_{\text {true }}-r\right|
$$

where two weights $\eta=0.05$ and $\mu=0.1$. The term associated with $\eta$ represents the shift of the center comparing to ground-truth circle. The term associated with $\mu$ represents the radius difference. The two weighting factor ensures that radius error is strongly weighted in comparison to center positions.

As suggested, Es less than 1 is considered as successful detection. Therefore, for weights $\eta=0.05$ and $\mu=0.1$ the greatest tolerance on center location could be up to 20 pixels in sum while radius error could be 10 pixels. On average, $(a, b, r)$ each has 10 pixels error tolerance. The statistics of error score Es are listed in Table 4-4. 
Table 4-4 Error score statistics

\begin{tabular}{|l|l|l|l|l|}
\hline Method & Max & Min & Mean & Standard deviation \\
\hline Geometric based & 2 & 0 & 0.51 & 0.32 \\
\hline Regular HT & 1.4 & 0 & 0.47 & 0.25 \\
\hline Modified HT & 1.2 & 0 & 0.45 & 0.24 \\
\hline AIS & 3.5 & 0 & 0.62 & 0.59 \\
\hline
\end{tabular}

The reason AIS based method has worse performance is that it detects more partial circles and arcs than the other three methods. For the other three methods, missing detection is not considered for error score calculation. Thus they have fewer samples and cannot fully show the performance difference.

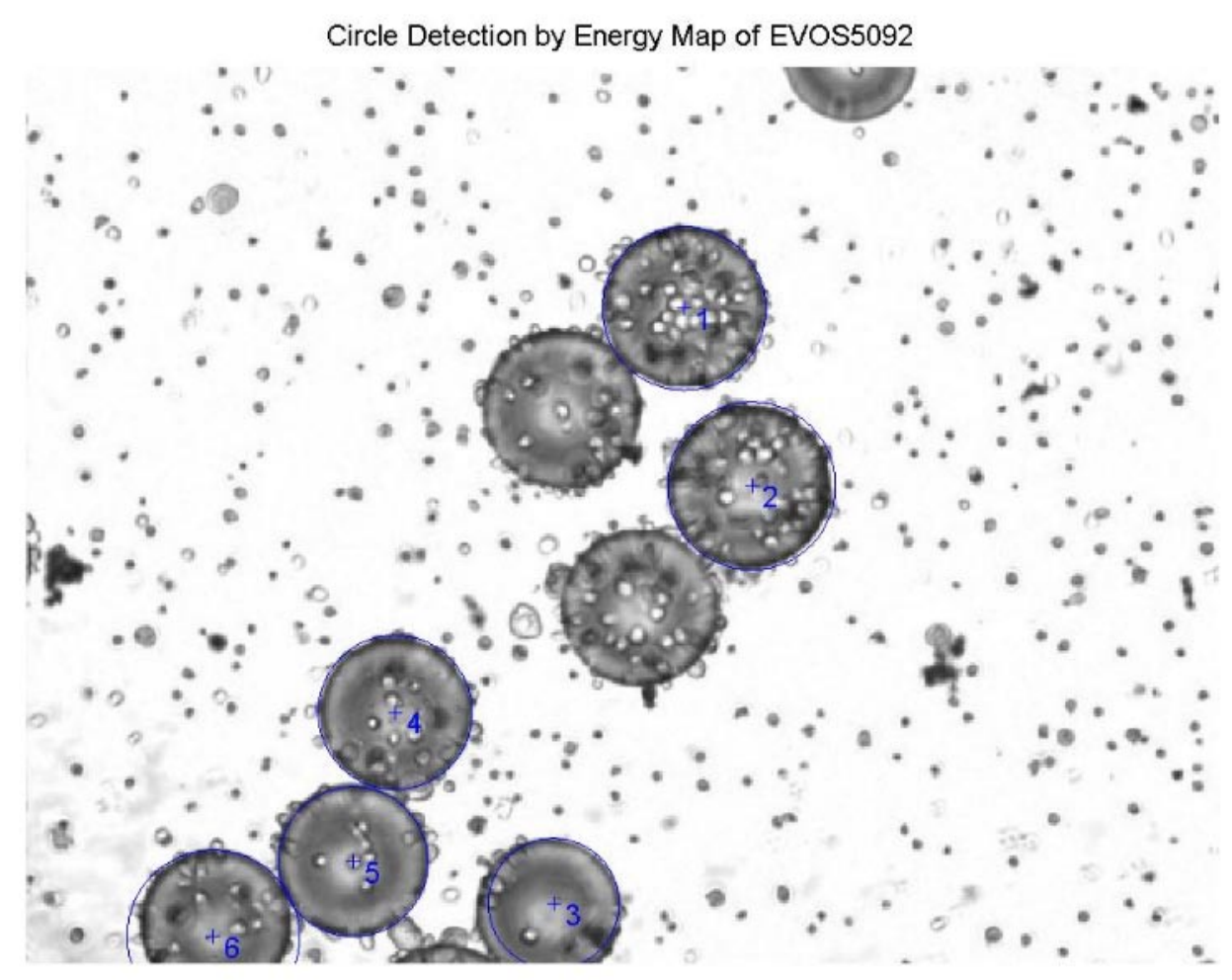

(a) 


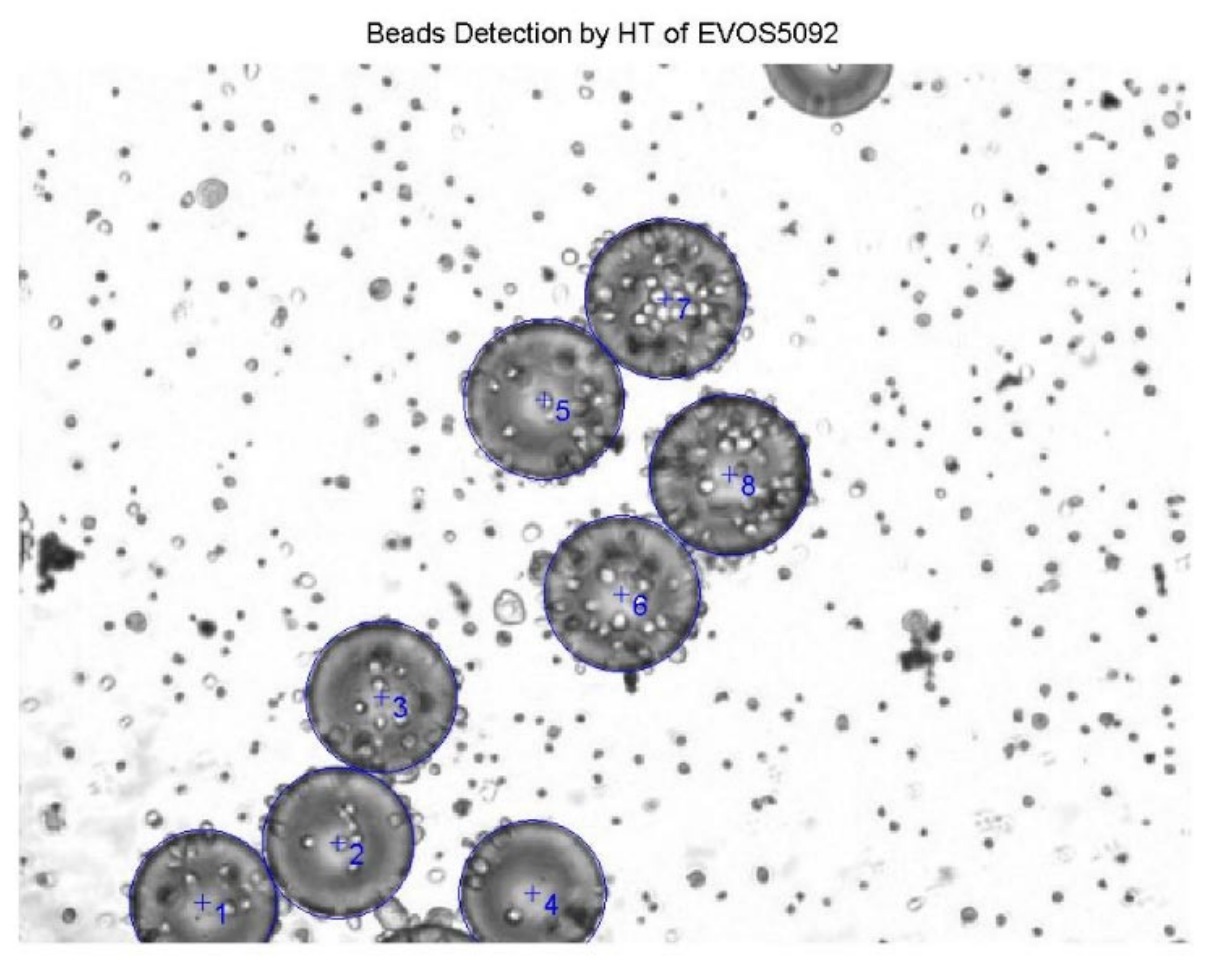

(b)

Final Detected Beads EVOS5092

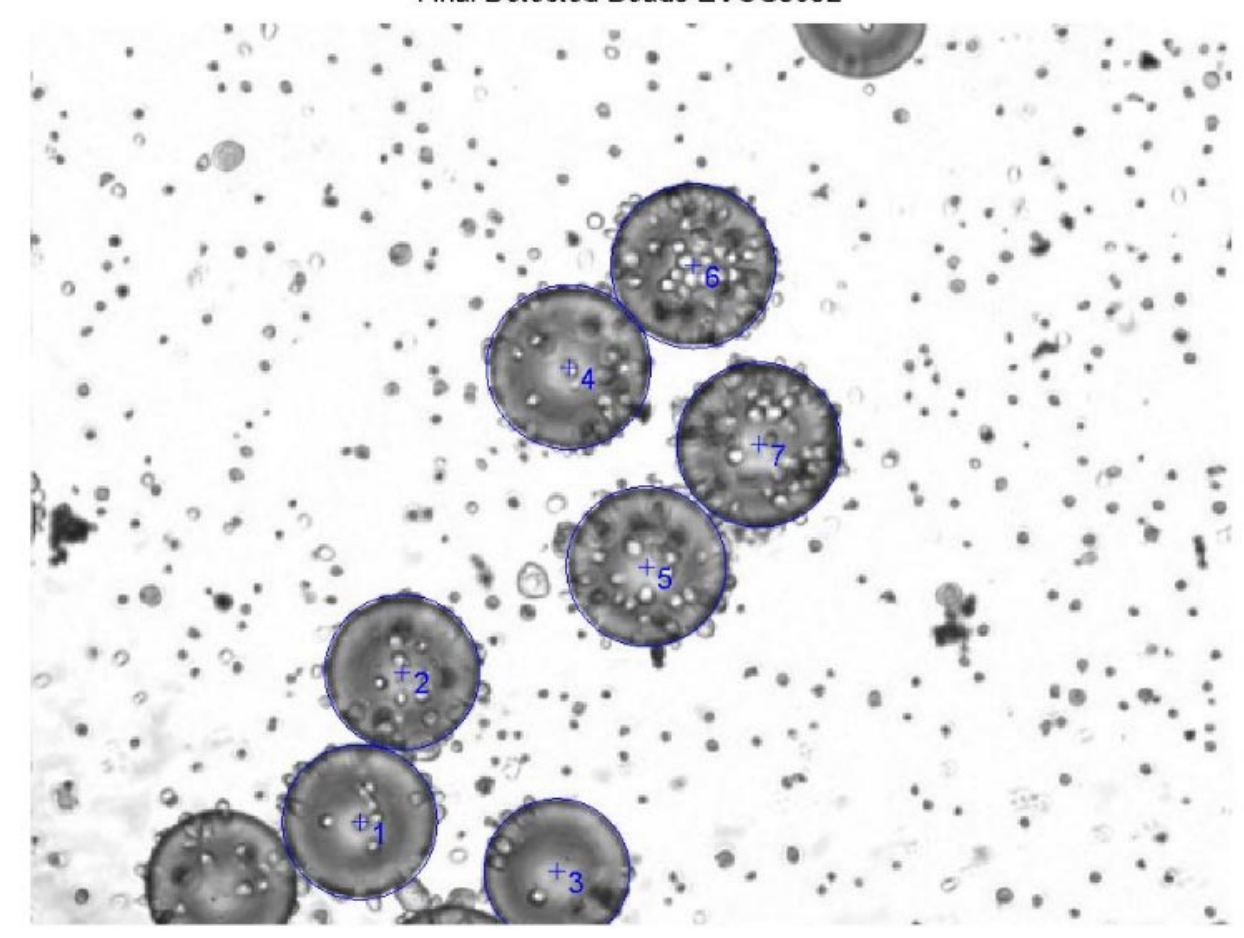

(c) 


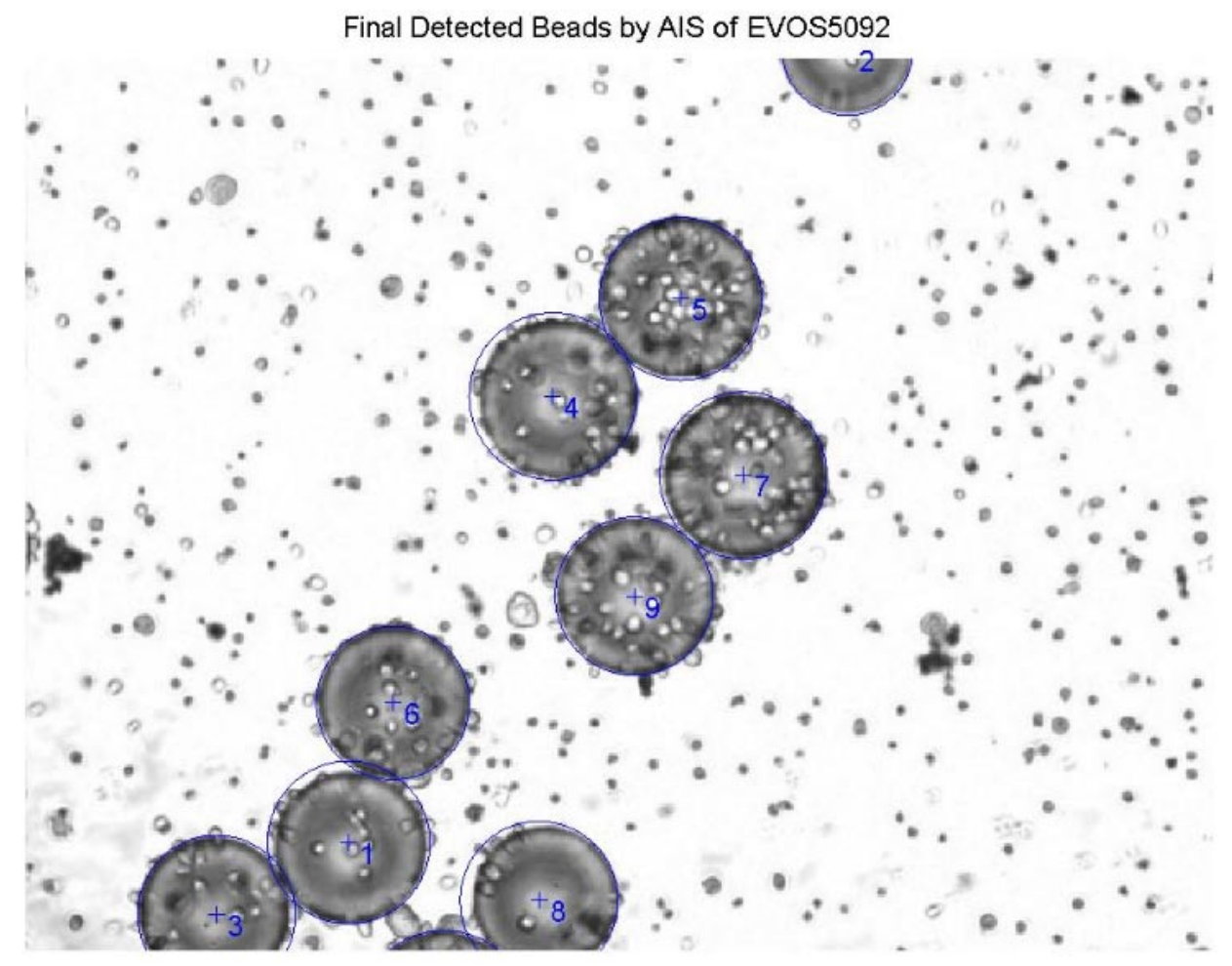

(d)

(a) By geometric feature based method, (b) by Hough Transform, (c) by modified Hough Transform, (d) by real valued artificial immune system

Figure 4.4 Circle detection results of image \#5092

In Figure 4.4, image (a) which is an example of geometric based energy map method has two bead missing detection in the middle area. Image (d) of AIS method is the only one has the partial bead near top of frame detected and labeled \#2.

In Figure 4.5 (c), Modified HT has the best performance with no false detection or missing detection. But in Figure 4.6 (b), regular HT has the worst result with 5 missing detections. 
Circle Detection by Energy Map of EVOS5121

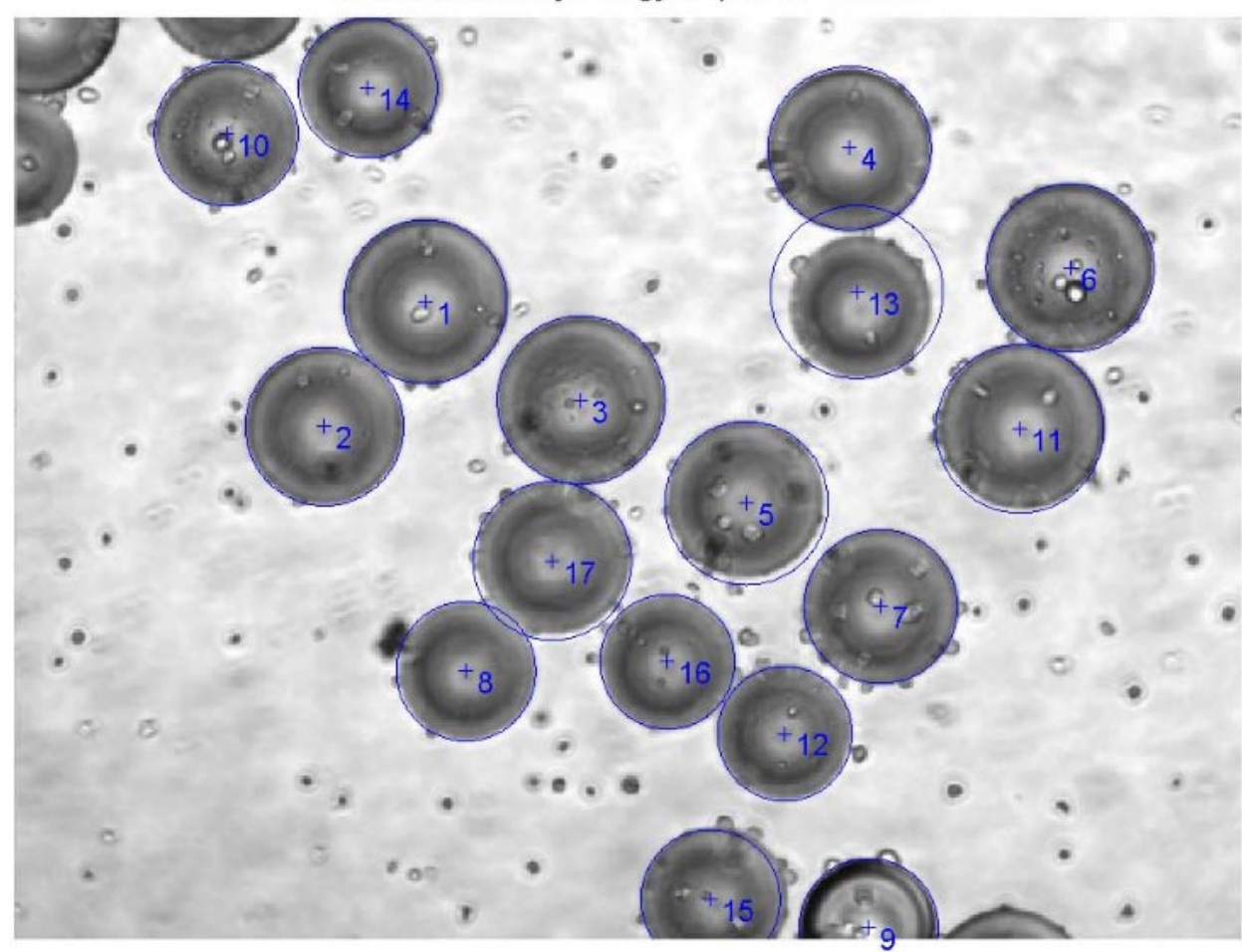

(a)

Beads Detection by HT of EVOS5121

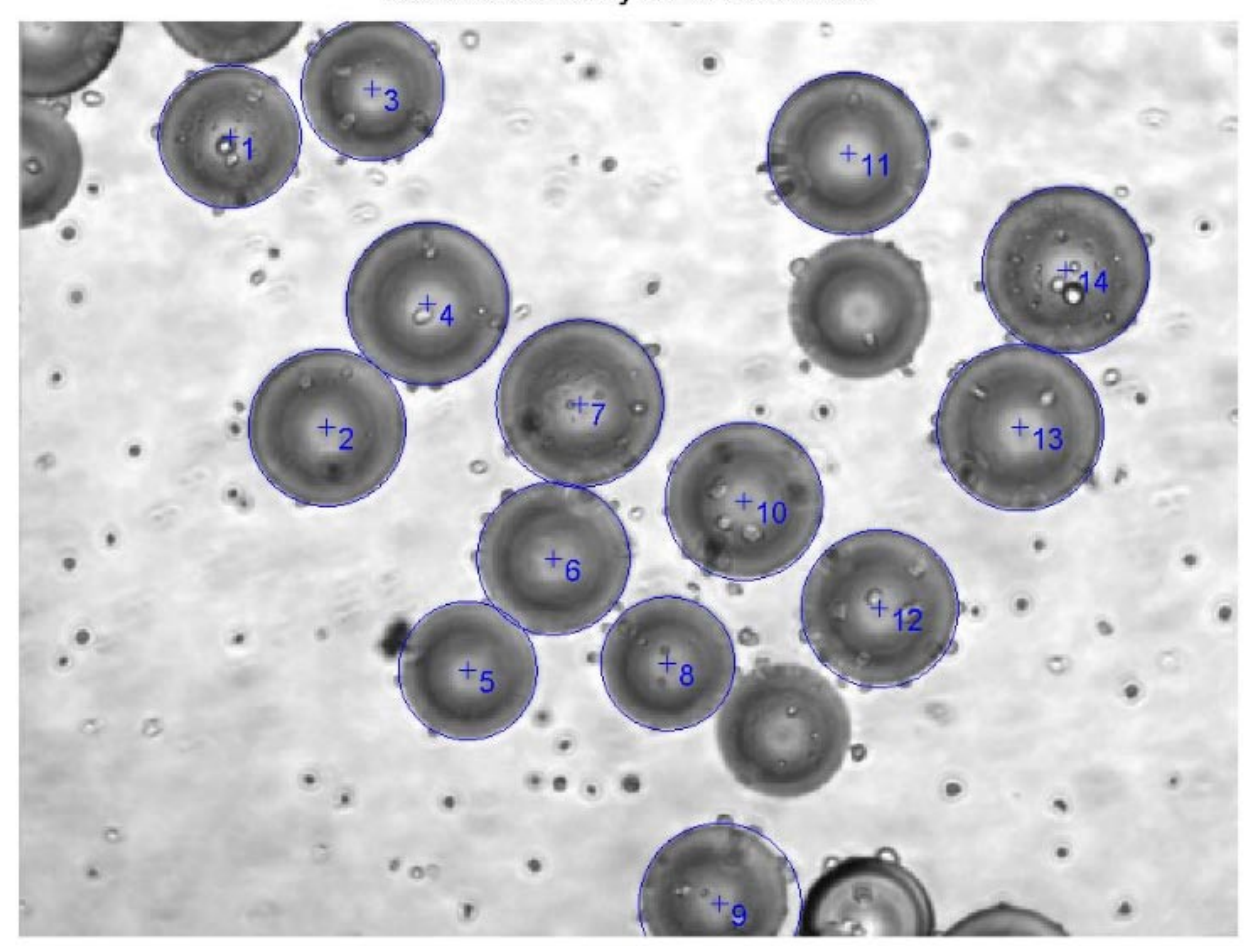

(b) 


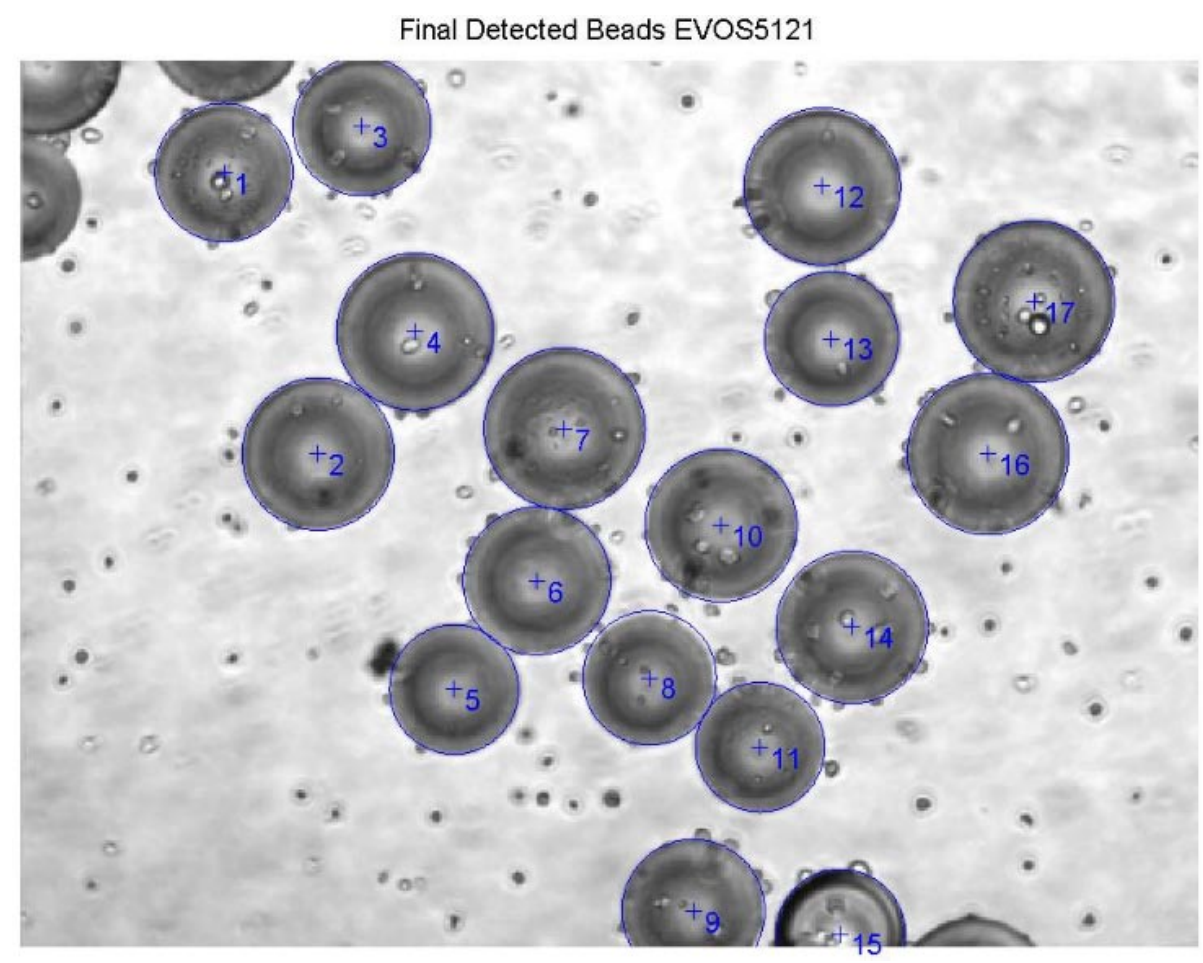

(c)

Final Detected Beads by AIS of EVOS5121

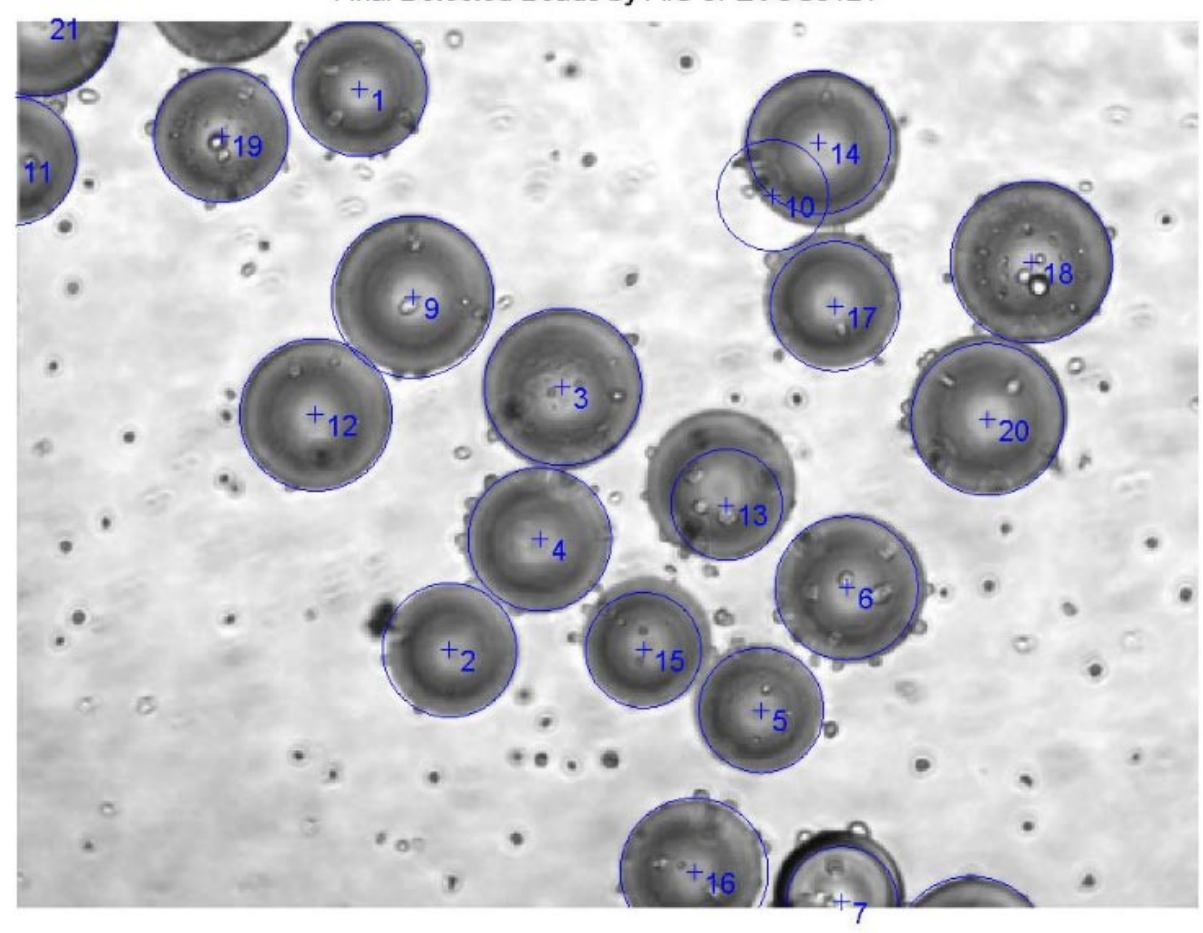

8

(a) By geometric feature based method, (b) by Hough Transform, (c) by modified Hough

Transform, (d) by real valued artificial immune system

Figure 4.5 Circle detection results of image \#5121 
Circle Detection by Energy Map of EVOS5133

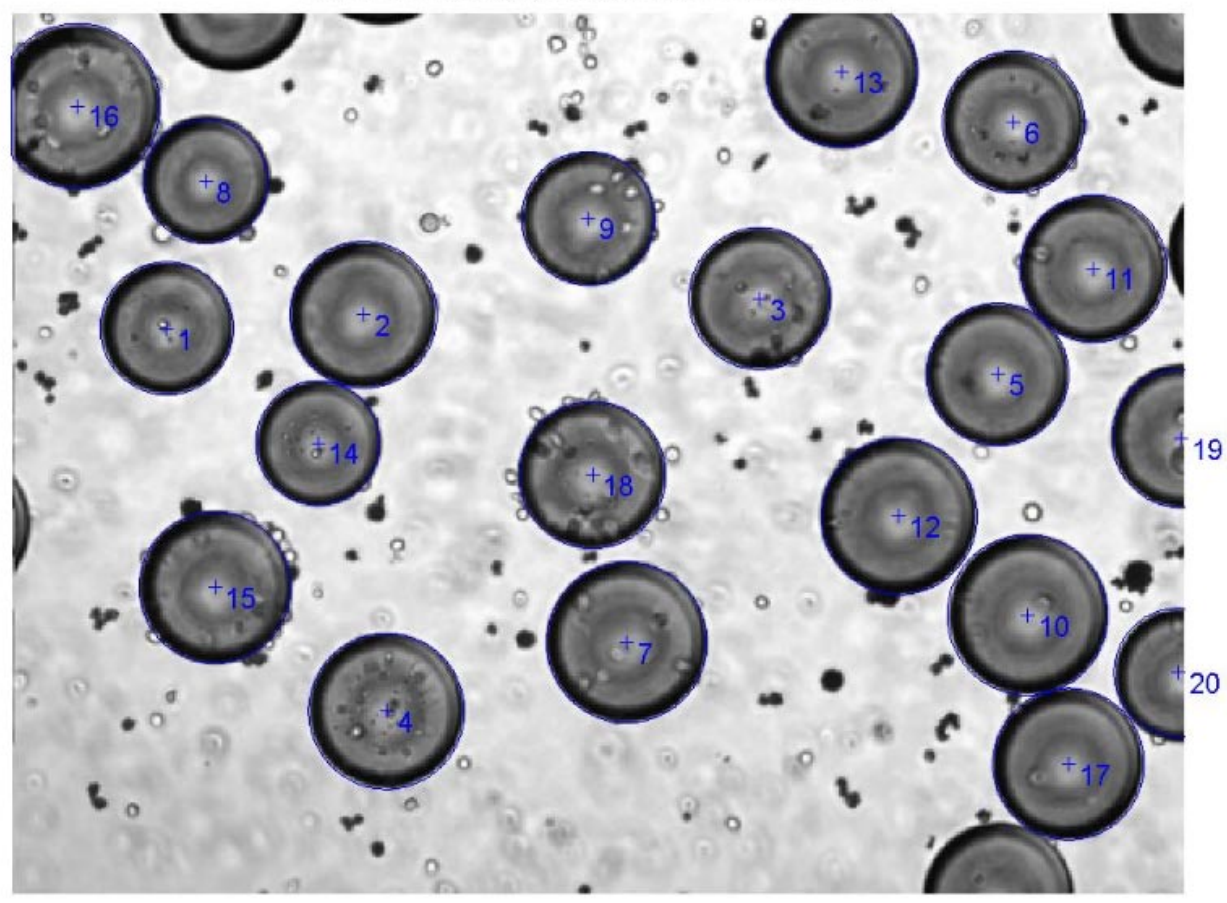

(a)

Beads Detection by HT of EVOS5133

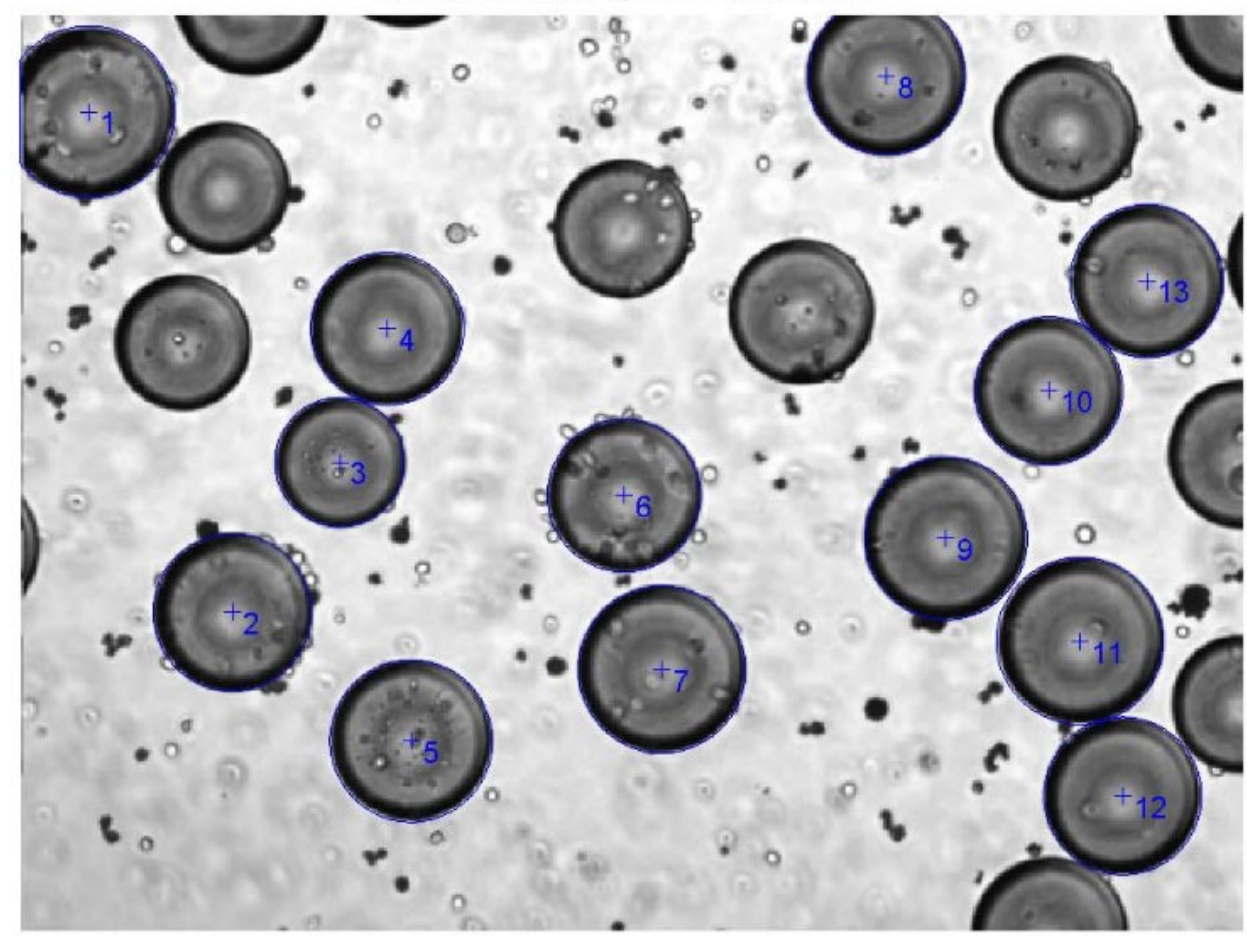

(b) 


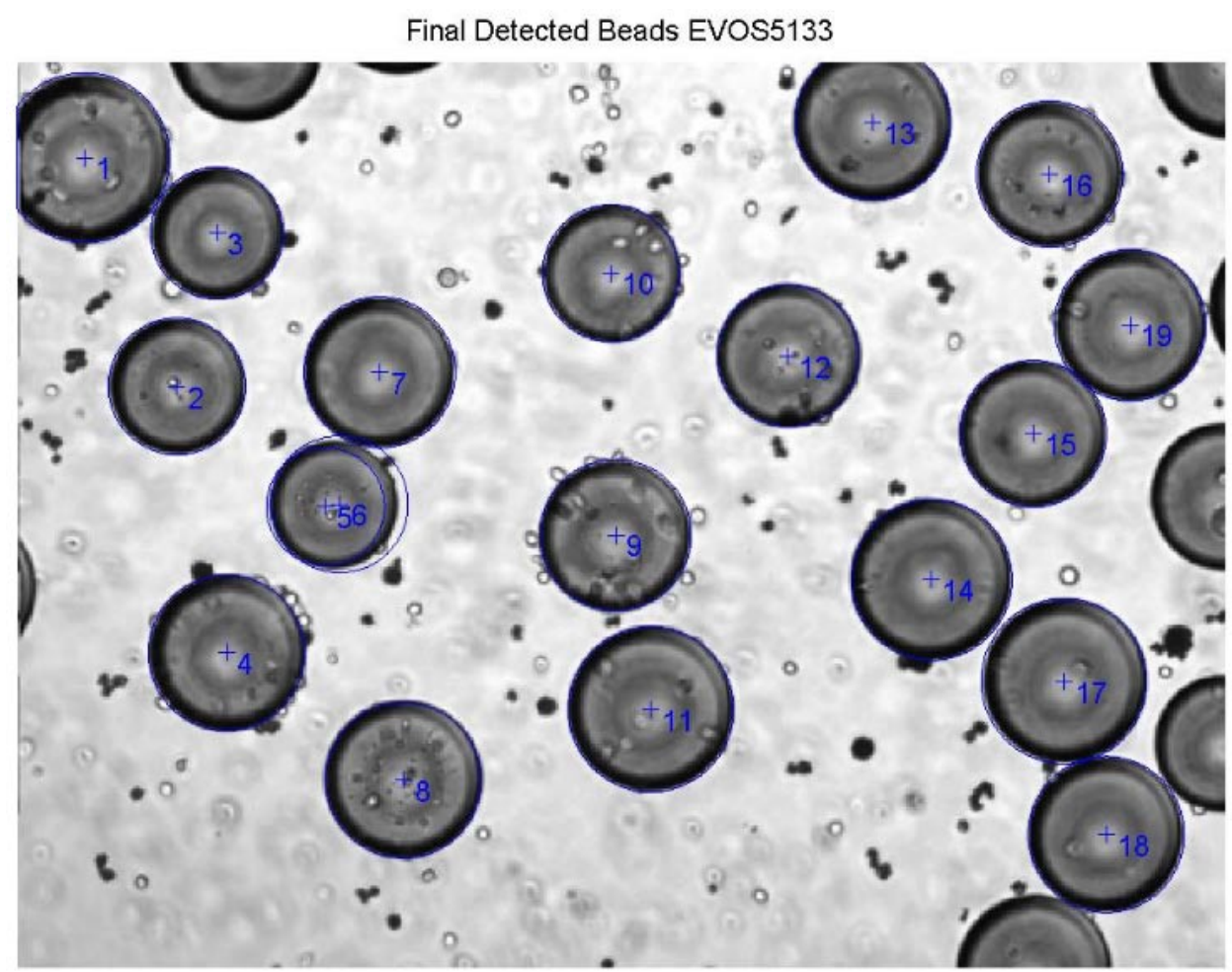

(c)

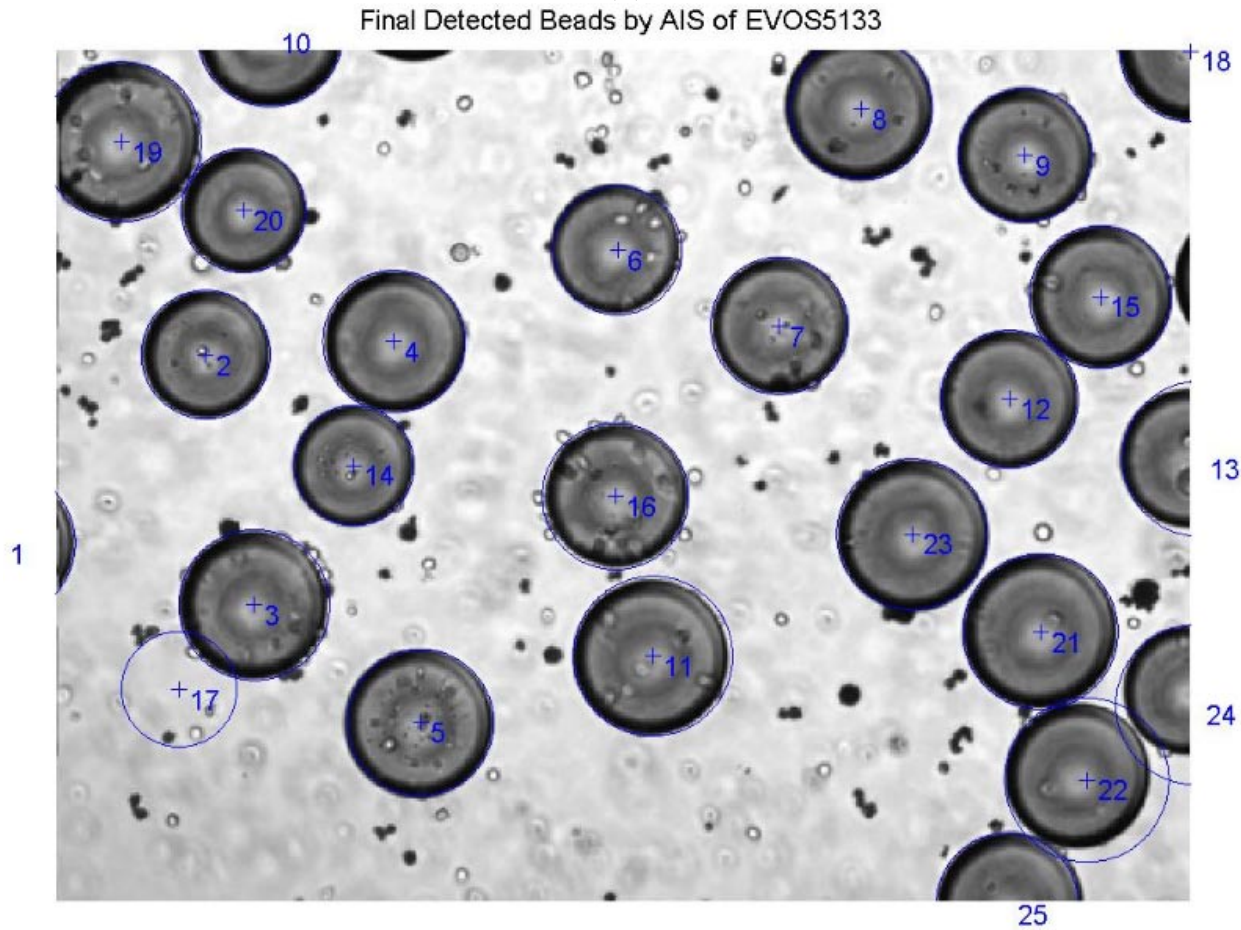

(d)

(a) By geometric feature based method, (b) by Hough Transform, (c) by modified Hough

Transform, (d) by real valued artificial immune system

Figure 4.6 Circle detection results of image \#5133 
Circle Detection by Energy Map of EVOS5169

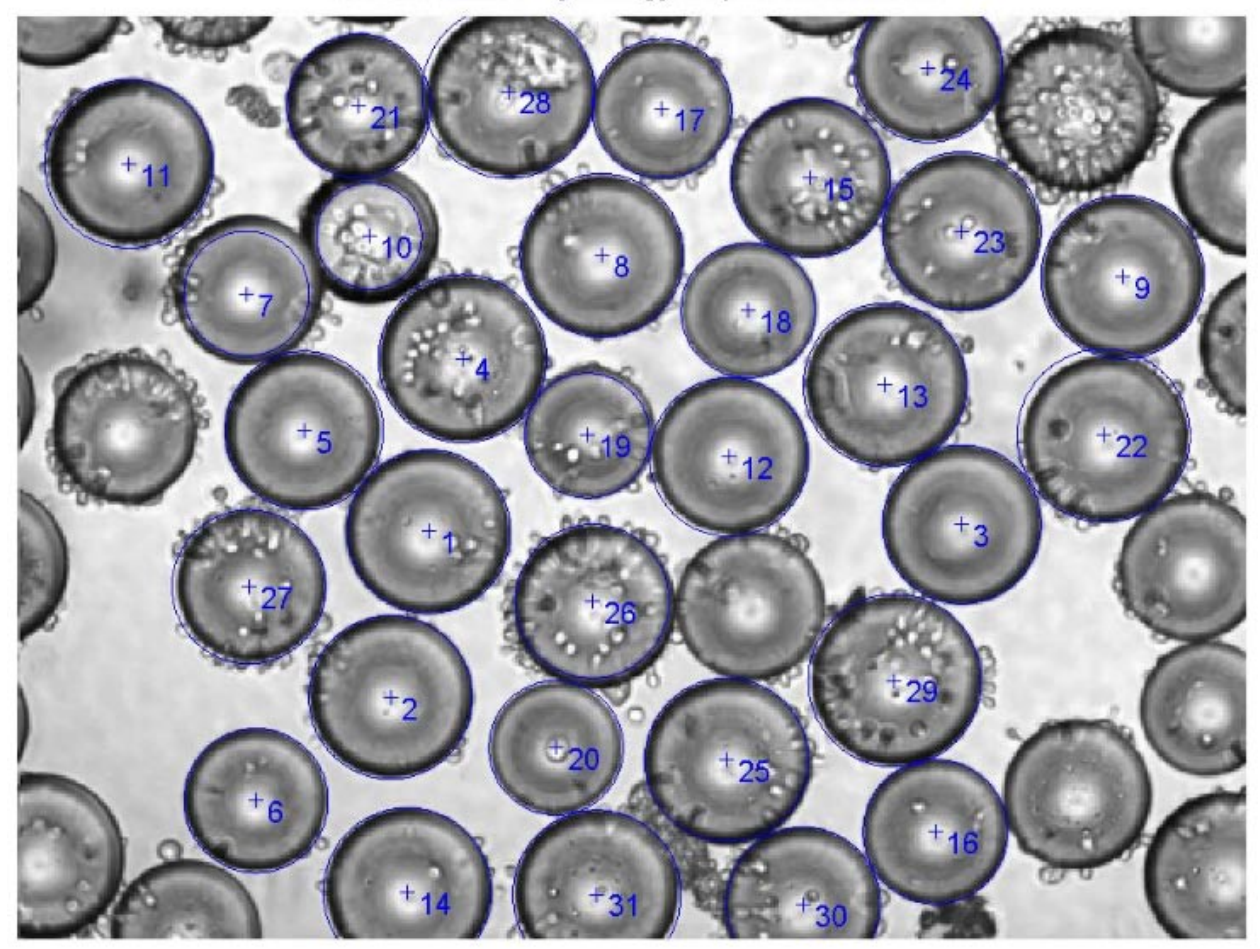

(a)

Beads Detection by HT of EVOS5169

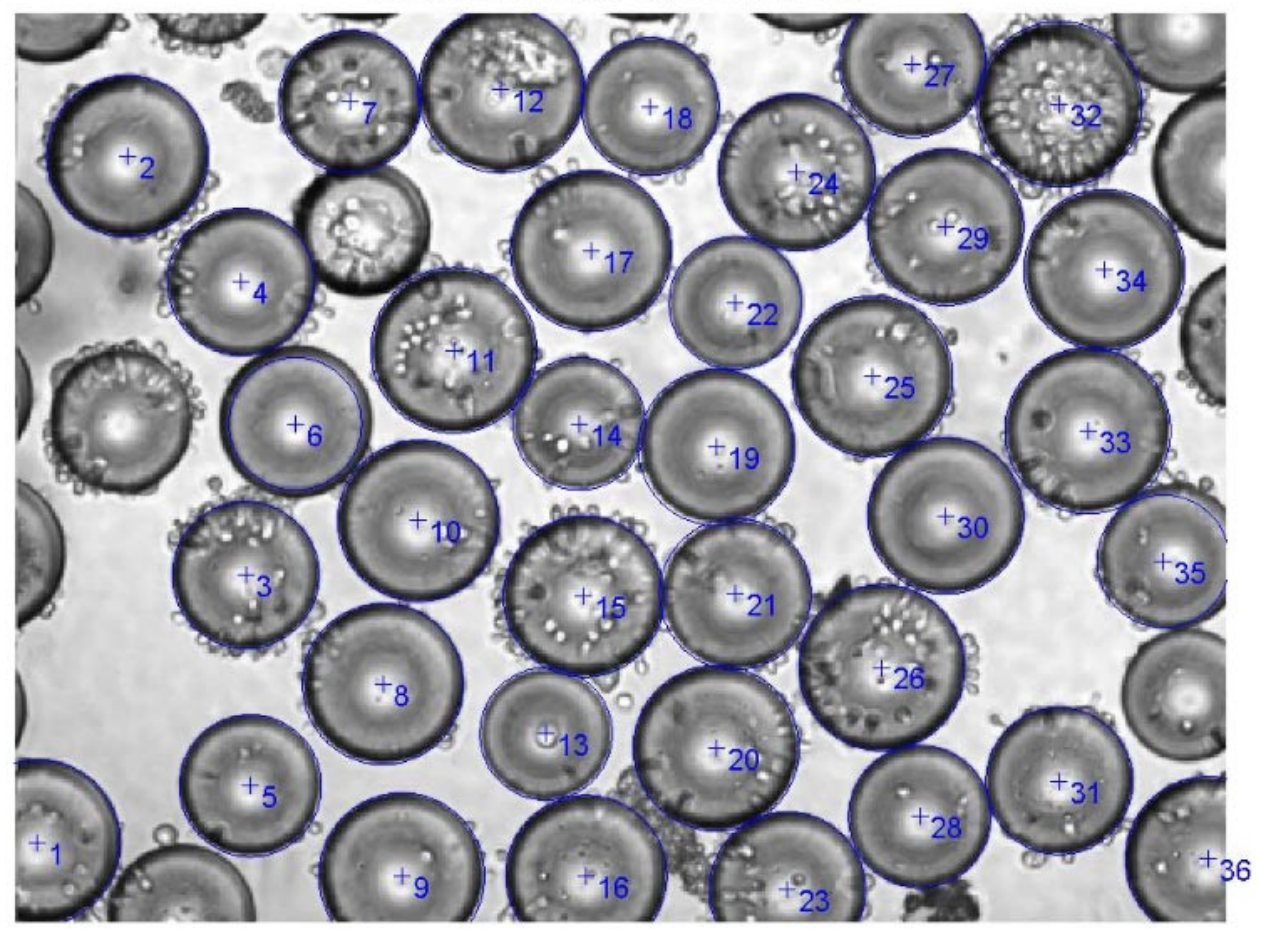

(b) 


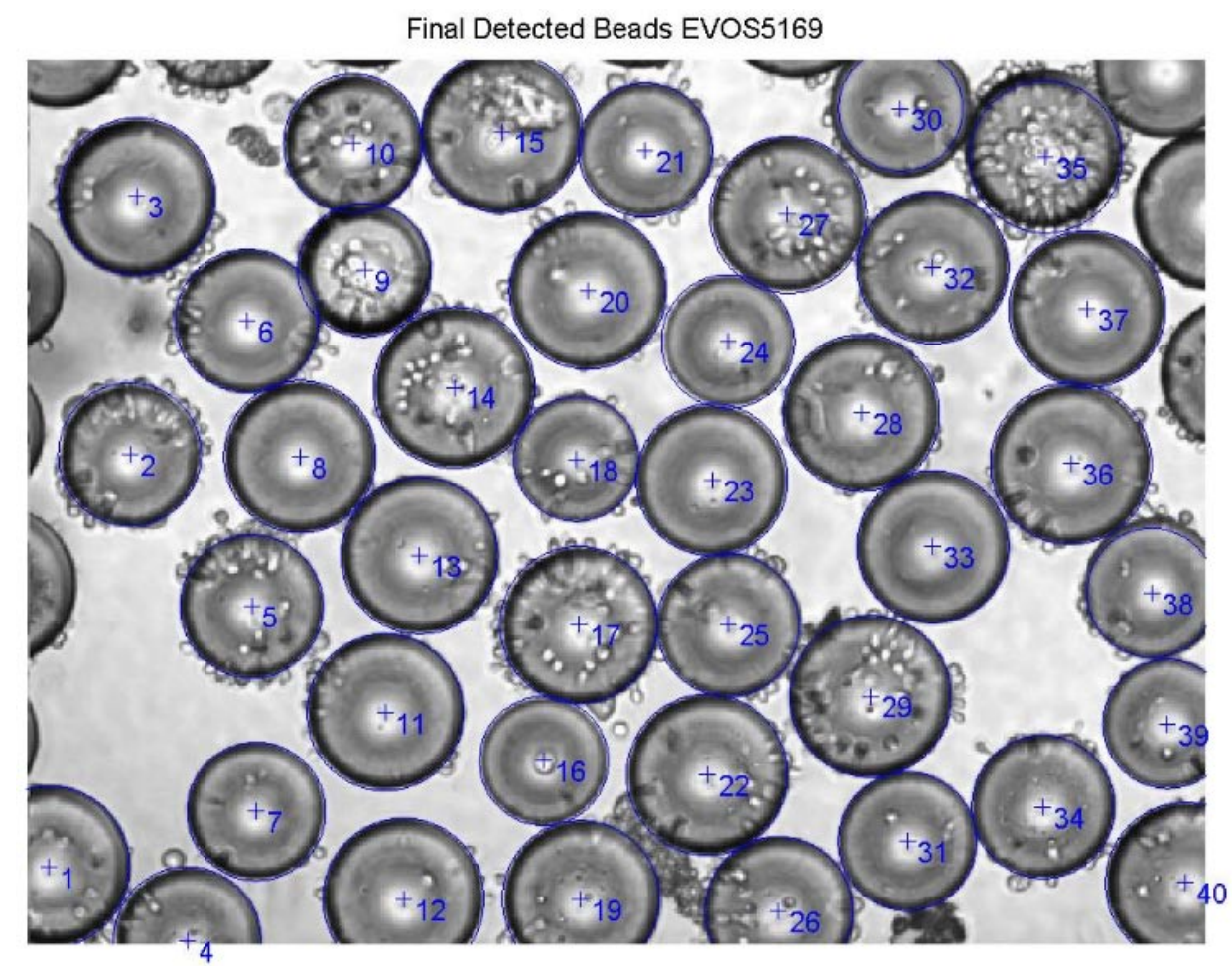

(c)

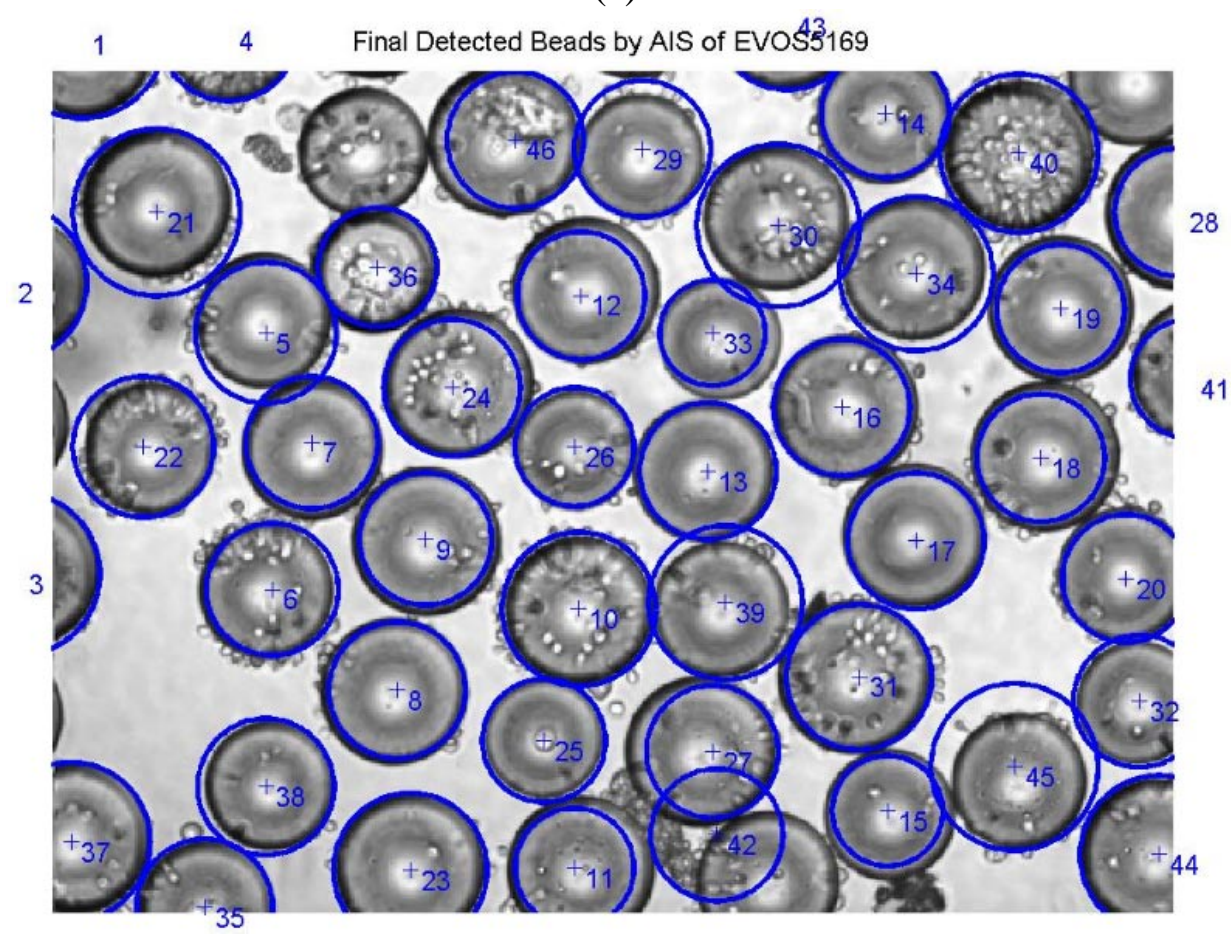

(d)

(a) By geometric feature based method, (b) by Hough Transform, (c) by modified Hough

Transform, (d) by real valued artificial immune system

Figure 4.7 Circle detection results of image \#5169 
It should be noticed that there is no absolute best method. Even new AIS based method does not have the best performance on every single sample seen from the detection results. For example, in Figure 4.5 (d) and Figure 4.7 (d), AIS based algorithm has more false detections than modified Hough Transform. But the Artificial Immune System based method has significant capability of detecting incomplete circles like arc pieces, while other methods could not collect enough number of data point to do accumulation and failed to detect those segments.

Another weakness of AIS method is that it does not always return the exact same results if repeated the program several times with the same image. Some differences could be seen from multiple run included different order of numbering of final detection, different false detections, or slightly inaccurate center locations and radii. In Figure 4.8 (d) if take a close look at the right bottom pictures which is an example of AIS based circle detection, one should be able to notice there is a very small imperfect circumference highlighting on left side of the traffic sign.

Natural image is also tested to evaluate the algorithms for other kinds of circular objects. In fact, most circular targets in natural environment are more or less artificial product made by men like wheels, traffic signs, disks and balls, and make them with very good circular appearance with edge detection. So the detection performance shows no significant difference when comparing to detecting cell bound beads. 


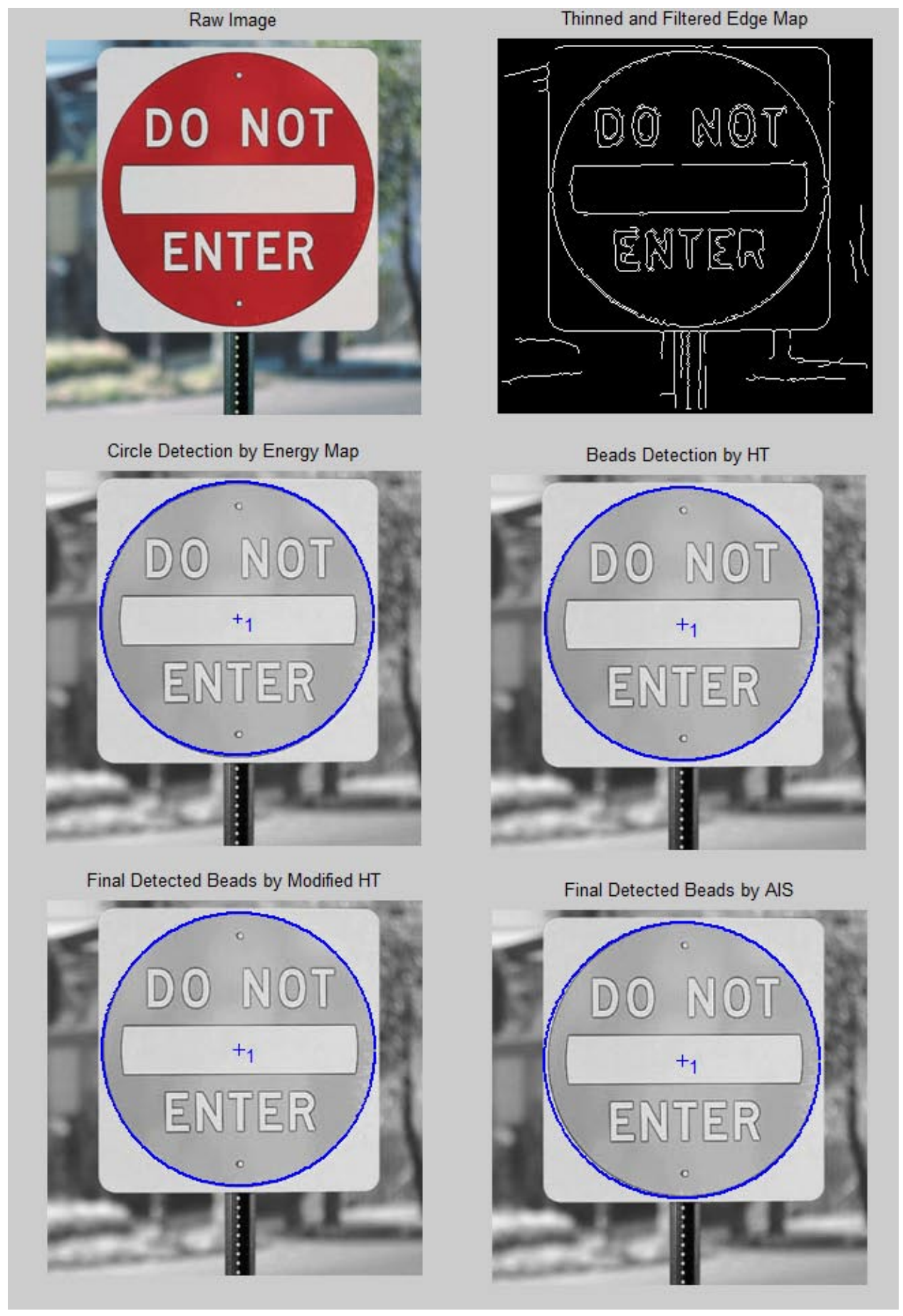

Figure 4.8 Example of traffic sign detection using four algorithms 


\section{CHAPTER 5 CONCLUSIONS AND FUTURE WORKS}

All four algorithms designed in this study are proven to possess certain level of abilities to locate beads from microscopic images and facilitate the automatic bladder cancer diagnosis. Through the test done with all images from the dataset, both deterministic modified Hough Transform and stochastic Artificial Immune System are able to achieve satisfying results. The advantages and disadvantage of each program are discussed next.

The geometric property based algorithm only utilizes circular shape's gradient information. It is the simplest algorithm to implement and processed very fast, any image from the dataset could be processed in less than 1 second on an Intel i5 CPU computer. But in this particular application where most beads edges are not perfect circular due to cells binding effect, the performance degrades severely. Straight lines along gradient direction could either be dispersed and hardly intersect, or tended to deviate from true center position. In Jia’s paper [22], all gradient orientated straight lines are 3-pixel wide instead of 1-pixel as in this study, in order to compromise incorrect gradient information and increase intersecting probability.

Hough Transform has been the most popular and very well developed approach on solving circle detection problems. It is very resistant to noises or imperfect shape appearance, because it is essentially a kind of mutual projection between image space and parameter space, based on circle definition function. The difficulty of Hough Transform is the huge amount of data in the parameter space during transformation. It not only takes a large amount of memory storage, but also makes local maxima finding very tough to handle. One way to tackle this is to quantize the parameter space, like combining 2-by-2 accumulation pixels into one single accumulation bin. This would smooth and enhance 
the accumulation in parameter space to some degree, but quantized parameter space cannot give the same resolution as image space resolution.

Due to the limitations, it is almost impossible to implement Hough Transform in real-time. Even though HT has been invented for nearly 50 years, researchers are still working on improving the efficiency of Hough Transform. The regular Hough Transform implemented in this study generally took a few minutes to process one image depending on the number of targets. This is not comparable to the other methods on the aspect of processing speed.

The modified Hough Transform in this study is a kind of combination of the first two methods. Its major advantage is that it reduces the number of parameter points generated from each image pixel from hundreds to only two. Strictly speaking it does not reduce the data in the parameter space because the parameter space is still the same size as regular Hough Transform, but the difference is most points are just zeros in the parameter space. Therefore, it does save a lot of processing time on deriving parameter data points and finding local maxima.

The Artificial Immune System based method adapts real valued Clonal Selection to circle detection problem. In contrast to the other binary encoded AIS approach in [74], the real valued AIS utilizes real valued vector representation for antibodies and introduces memory cells to store detected circles along with the processing. There are several advantages with real value implementation.

First, only a small number of antigens are required. The initialization and mutation are all in real value domain and being constrained on real existing edge segments, thus it is the same number of independent edge segments within edge map. In 
binary antibody representation, the pixel coordinates are converted to binary strings for clonal selection and mutation, and then converted back to real value of center and radius to check if they have real existing edge pixel correspondents. It could not assure new antibodies' placement on edge pixels, hence many antibodies generated during the iterations are actually wasted when they are placed on black background. The binary representation requires hundreds of antibodies and iterations to detect a few circle objects in image.

Second, by introducing memory cells to store detected circle during the iterations, this program can output all detected results once clonal selection is finished. This saves more time and memory space comparing to maintain all iteration records and go back for local maxima finding after clonal selection procedure. A small threshold would be necessary to validate antibody's affinity with edge antigens and pick out successful detection for memory cells. However, this threshold is not influential to good detection, because the chance of having complete overlapping circles is very small, unless their centers are very close to each other. In contrast, the binary encoded AIS approach's memory is used to keep all antibodies throughout entire processing iterations.

Third, the immunological process of antibodies destroying antibodies is simulated by the operation of removing detected circle through the iterations. Thus through the program iterations, less and less antibodies are need and further reduced the requirement of repetition times and storage space. 
Table 5-1 Comparison of four circle detection algorithms

\begin{tabular}{|l|l|l|}
\hline & PROS & CONS \\
\hline $\begin{array}{l}\text { Geometric property } \\
\text { based }\end{array}$ & $\begin{array}{l}\text { 1) Easy to implement, } \\
\text { 2) Fastest. }\end{array}$ & $\begin{array}{l}\text { 1) Sensitive to noise and } \\
\text { deformation, } \\
\text { 2) Hard to tune } \\
\text { parameters. }\end{array}$ \\
\hline $\begin{array}{l}\text { Regular Hough } \\
\text { Transform }\end{array}$ & $\begin{array}{l}\text { 1) Resistant to noise and } \\
\text { deformation, } \\
\text { 2) Accurate center and } \\
\text { radius detection. }\end{array}$ & $\begin{array}{l}\text { 1) Slow to process, } \\
\text { 2) Very large memory } \\
\text { space required, } \\
\text { 3) Hard to implement, } \\
\text { 4) Hard to tune } \\
\text { parameters. }\end{array}$ \\
\hline $\begin{array}{l}\text { Modified Hough } \\
\text { Transform }\end{array}$ & $\begin{array}{l}\text { 1) Faster, } \\
\text { 2) Resistant to noise and } \\
\text { deformation, } \\
\text { 3) Accurate detection. }\end{array}$ & $\begin{array}{l}\text { 1) Hard to tune } \\
\text { parameters, } \\
\text { 2) Still require large } \\
\text { memory. }\end{array}$ \\
\hline $\begin{array}{l}\text { Real valued Artificial } \\
\text { Immune System }\end{array}$ & $\begin{array}{l}\text { 1) Faster, } \\
\text { 2) Resistant to noise and } \\
\text { deformation, } \\
\text { 3) Small memory required, } \\
\text { 4) Easy to implement, } \\
\text { 5) Easy to tune parameters. }\end{array}$ & $\begin{array}{l}\text { 1) Slightly inconsistent } \\
\text { runs. }\end{array}$ \\
\hline
\end{tabular}

In this study four circle detection algorithms are implemented and adapted to the task of automatic bladder cancer diagnosis. Different programs of deterministic methods such as geometric based, Hough Transform and its variation, and Computational Intelligence models such as Genetic Algorithm and Artificial Immune System, are designed and tested on the microscopic image dataset. It is very interesting to see the power of biological inspired method on solving complex pattern recognition problems. Its accuracy and robustness have great potentials in the field of processing natural images.

This study is a successful attempt to adapt Artificial Immune System to the hot topic of circle detection problem in image processing field. Further research could be targeting on making use of other Artificial Immune System model like Negative 
Selection on circle detection, or extending the real valued Clonal Selection model to recognition problems of other primitive shapes.

The contribution of this study is for the first part of the entire cancer cell grading system, which is locating the ROI for cancer cell binding analysis. The second part is to analyze the cancer cell binding condition for each bead separately in the microscopic image.

Cell segmentation is another practical task in image processing. It can be completed based on the shape of a cell like circle or eclipse, or based on some color feature like darker nucleus area of a cell. In this dataset, due to the half transparent appearance of cancer cells, a segmentation method based on intensity level may work better than color based method just like edge information is chosen for bead detection.

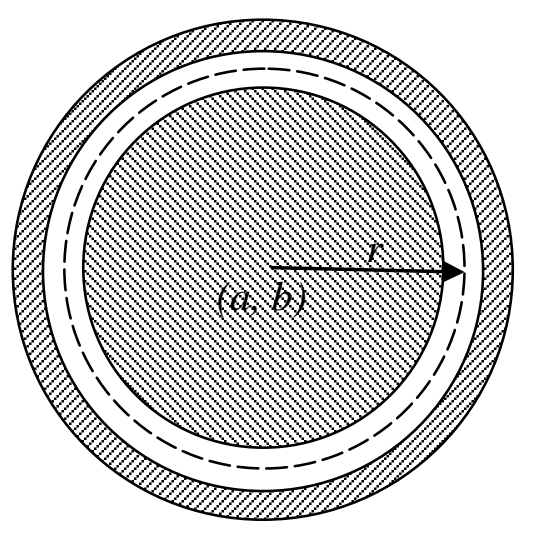

Figure 5.1 Regions for cell binding analysis

But the most important part is to analyze cells both on the circle boundary and within circle boundary. In Figure 5.1, the dashed circumference represents detected bead from this study, then the shaded area of a disk and a ring is for cell detection. Due to reflection 
effect, all beads in images have a wide dark contour. The white area around dashed line represents this contour and should be ignored.

After all cancer cells are segmented from the shaded area, the ratio of cells coverage on bead can be calculated through dividing the total area of cells by total shaded area. For better accuracy, two weighting factors can be added to combine disk area ratio and ring area ratio. When every bead's cell binding condition is acquired, the illness level of bladder cancer can be generalized from averaging all beads grades. To this point, the automatic bladder cancer diagnosis based on PLZ4 smart bead shall be completed.

\begin{tabular}{|l|l|}
\hline Binding area & Grade \\
\hline $0 \%-20 \%$ & 1 \\
\hline $20 \%-40 \%$ & 2 \\
\hline $40 \%-60 \%$ & 3 \\
\hline $60 \%-80 \%$ & 4 \\
\hline $80 \%-100 \%$ & 5 \\
\hline
\end{tabular}

Table 5-2 Grading rule for single bead's cell binding condition 


\section{REFERENCES}

[1] American Cancer Society, “Cancer Facts \& Figures 2012”, American Cancer Society Inc., 2012, pp. 22.

[2] W. H. Nailon, A. T. Redpath and D. B. McLaren, "Texture analysis of 3D bladder cancer CT images for improving radiotherapy planning”, Proceedings of 5th IEEE International Symposium on Biomedical Imaging: From Nano to Macro, May 2008, pp. 652-655.

[3] G. Begelman, E. Rivlin, “Automatic screening of bladder cells for cancer diagnosis”, Proceedings of 2009 16th IEEE International Conference on Image Processing, Nov. 2009, pp. 673-676.

[4] G. Karemore, M. Nielsen, K. K. Mascarenhas, K. S. Choudhary, A. Patil, V. K. Unnikrishnan, V. Prabhu, A. Chowla and C. Santhosh, "Classification of Laser Induced Fluorescence spectra from normal and malignant tissues using Learning Vector Quantization neural network in bladder cancer diagnosis”, Proceedings of 8th IEEE International Conference on BioInformatics and BioEngineering, Oct. 2008, pp. $1-6$.

[5] M. A. V. Rapsomaniki, P. G. Zerefos, K. A. Theofilatos, S. D. Likothanassis, A. K. Tsakalidis and S. P. Mavroudi, "A novel pipeline method for the preprocessing of Mass spectrometry proteomics data”, Proceedings of 2010 10th IEEE International Conference on Information Technology and Applications in Biomedicine, Nov. 2010, pp. 1-4.

[6] C. Cheng-Hsin, W. Ching-Hua, H. You-Ming, H. Huei-Sheng and H. Fei-Bin, "Impedance sensing of bladder cancer cells based on a single-cell-based DEP microchip”, Proceedings of 2009 IEEE Sensors Conference, Oct. 2009, pp. 943-947.

[7] C. Moallemi, “Classifying cells for cancer diagnosis using neural networks", IEEE Expert, vol.6, pp. 8-12, 1991.

[8] Y. Hu, K. Ashenayi, R. Veltri, G. O'Dowd, G. Miller, R. Hurst and R. Bonner, “A comparison of neural network and fuzzy c-means methods in bladder cancer cell classification”, Proceedings of 1994 IEEE International Conference on Neural Networks, Jul. 1994, vol.6.pp. 3461-3466.

[9] A. F. Said, L. J. Karam, M. E. Berens, Z. Lacroix and R. A. Renaut, "MIGRATION AND PROLIFERATION ANALYSIS FOR BLADDER CANCER CELLS”, Proceedings of 4th IEEE International Symposium on Biomedical Imaging, April 2007, pp. 320-323.

[10] Tzu-yin Lin, Hongyong Zhang, Sisi Wang, Li Xie, Bin Li, Carlos O Rodriguez Jr, Ralph de Vere White, Chong-xian Pan, “Targeting canine bladder transitional cell 
carcinoma with a human bladder cancer-specific ligand”, Molecular Cancer, Jan 2011, 10:9, pp. 1-6.

[11] Hongyong Zhang, Olulanu H. Aina, Kit S. Lam, Ralph de Vere White, Christopher Evans, Paul Henderson, Primo N. Lara, Xiaobing Wang, James A. Bassuk, Chongxian Pan, "Identification of a bladder cancer-specific ligand using a combinatorial chemistry approach”, Urologic Oncology: Seminars and Original Investigations, Oct 2010, pp. 1-11.

[12] Zhou Zhifeng, "Measuring Diameter of Non-threaded Hex Bolts Based on Hough Transform”, Proceedings of 2011 Third International Conference on Measuring Technology and Mechatronics Automation, Jan. 2011, vol.2, pp. 526-528.

[13] Liya Thomas, Lamine Mili, “A Robust GM-Estimator for the Automated Detection of External Defects on Barked Hardwood Logs and Stems”, IEEE Transactions on Signal Processing, vol. 55, no.7, pp. 3568-3576.

[14] Jens Lundstrom, Antanas Verikas, "Detecting halftone dots for offset print quality assessment using soft computing”, Proceedings of 2010 IEEE International Conference on Fuzzy Systems, pp. 1-7.

[15] N. Fujiwara, T. Onda, M. Niwakawa, "Three-dimensional circle detection and location of pipe joints for bin-picking tasks”, Proceedings of 1998 IEEE/RSJ International Conference on Intelligent Robots and Systems, Oct 1998, vol.2, pp. 1216-1221.

[16] Ai-Shan Chiang, Po-Ting Liu, Yi-Ping Lien, Yu-Len Huang, Tsu-Yi Hsieh, "Automatic detection of antinuclear autoantibodies cells in indirect immunofluorescence images”, Proceedings of 2010 3rd International Conference on Biomedical Engineering and Informatics, Oct. 2010, vol. 1, pp. 137-140.

[17] S.H. Peckinpaugh, R.J. Holyer, "Circle detection for extracting eddy size and position from satellite imagery of the ocean", Proceedings of IEEE Transactions on Geoscience and Remote Sensing, Mar 1994, vol.32, no.2, pp. 267-273.

[18] Hongmin Zhang, Chenying Liang, Yan Wang, “Chord midpoint randomized hough transform for the cell image segmentation”, Proceedings of 2011 Cross Strait QuadRegional Radio Science and Wireless Technology Conference, vol.2, pp. 1446-1450.

[19] M. K. Leung, T. S. Huang, "Detecting wheels of vehicle in stereo images", Proceedings of 10th International Conference on Pattern Recognition, 1990, vol.1, pp. 263-267.

[20] D. Young, C.A. Glasbey, A.J. Gray, N.J. Martin, "Identification and sizing of cells in microscope images by template matching and edge detection”, Proceedings of Fifth 
International Conference on Image Processing and its Applications, 1995, pp. 266270.

[21] J.P. Lewis, “Fast Template Matching”, Vision Interface 95, May1995, pp. 120-123.

[22] Li-Qin Jia, Hong-Min Liu, Zhi-Heng Wang, Hong Chen, "An effective non-HT circle detection for centers and radii”, Proceedings of 2011 International Conference on Machine Learning and Cybernetics, July 2011, vol.2, pp. 814-818.

[23] J. A. Canny, “A Computational Approach to Edge Detection”, IEEE Transaction on Pattern Analysis and Machine Intelligence, Nov. 1986, vol.8, no.6, pp. 679-698.

[24] H.K. Yuen, J. Princen, J. Illingworth, J. Kittler, “A Comparative Study of Hough Transform Methods for Circle Finding”, Image and Vision Computing - Special issue: 5th Alvey vision meeting, Feb 1990, pp. 169-174.

[25] Fei Shang, Jinwei Liu, Xiao Zhang, Di Tian, “An improved circle detection method based on right triangle inscribed in a circle", Proceedings of 2009 WRI World Congress on Computer Science and Information Engineering, 2009, vol.6, pp. 382387.

[26] Shang Fei, Wang Fenggui, Tian Di, Zhao Zhihui, "A method for circle detection based on right triangles inscribed in a circle", Acta Optica Sinica, 28(4), 2008, pp.739-743.

[27] Heung-Soo Kim, Jong-Hwan Kim, "A two-step circle detection algorithm from the intersecting chords”, Pattern Recognition Letters, May 2001, vol.22, pp. 787-798.

[28] P.V.C. Hough, “A method and means for recognizing complex patterns”, U.S. Patent 3,069,654. 1965.

[29] R. Duda, P. E. Hart, "Use of the Hough Transform to Detect Lines and Curves in Pictures”, Communications of ACM, 1972, vol.15, no.1, pp. 11-15.

[30] P. E. Hart, "How the Hough transform was invented [DSP History]", IEEE Signal Processing Magazine, Nov 2009, vol.26, no.6, pp.18-22.

[31] J. ILLINGWORTH AND J. KITTLER, "A Survey of the Hough Transform", COMPUTER VISION, GRAPHICS, AND IMAGE PROCESSING 44, 1988, pp. 87116.

[32] C. Kimme, D. Ballard, J. Sklansky, "Find circles by an array of accumulators", Communication of the ACM, 1975, wol.18, no.2, pp. 120-122.

[33] Davies E. R., “A modified Hough scheme for general circle location”, Pattern Recognition Letters, 1988, vol.7, no.1, pp. 36-44. 
[34] D.H. Ballard, "Generalizing the Hough Transform to Detect Arbitrary Shapes", Pattern Recognition, 1981, vol.13, no.2, pp. 111-122.

[35] J. Illingworth, J. Kittler, "The adaptive Hough Transform”, IEEE Transactions on Pattern Analysis and Machine Intelligence, Sep 1987, no.5, pp. 690-697.

[36] J. Illingworth, J. Kittler, J. Princen, "Shape detection using the adaptive Hough Transform", Proceedings of the NATO Advanced Research workshop on Real-time object measurement and classification, Sep 1987.

[37] T.J. Atherton, "Using phase to represent radius in the coherent circle Hough transform”, IEEE Colloquium on Hough Transforms 5, May 1993, pp. 1-4.

[38] T. J. Atherton, D. J. Kerbyson, “The coherent circle Hough Transform”, Proceedings of the British Machine Vision Conference, 1993, pp. 269-278.

[39] T. J. Atherton, D. J. Kerbyson, "Size invariant circle detection”, Image and Vision Computing, 1999, vol.17, no.11, pp. 795-803.

[40] D. J. Kerbyson, T. J. Atherton, "Circle detection using Hough transform filters", Proceedings of Fifth International Conference on Image Processing and its Applications, 1995, pp. 370-374.

[41] R.K.K. Yip, D.N.K. Leung , S.O. Harrold, "Line segment patterns Hough transform for circles detection using a 2-dimensional array", Proceedings of the International Conference on Industrial Electronics, Control, and Instrumentation, Nov 1993, vol.3, pp. 1361-1365.

[42] J. Illingworth, J. Kittler, "A survey of efficient Hough Transform methods”, Proc. 3rd Alvey Vision Conference, 1987, pp. 319-326.

[43] H.K.Yuen, J. Princen, J. Illingworth, J. Kittler, "A comparative study of Hough transform methods for circle finding", Image and Vision Computing - Special issue: 5th Alvey vision meeting, Feb 1990, vol.8, no.1, pp. 169-174.

[44] Dimitrios Ioannoua, Walter Hudab, Andrew F. Lainec, "Circle recognition through a 2D Hough Transform and radius histogramming", Image and Vision Computing, Jan 1999, vol.17, no.1, pp. 15-26.

[45] P. Kultanen, L. Xu, E. Oja, "Randomized Hough transform”, Proceedings of 10th International Conference on Pattern Recognition, Jun 1990, vol.1, pp. 631-635.

[46] M.H.D.H. Tooei, J.R. Mianroodi, N. Norouzi, A. Khajooeizadeh, "An innovative implementation of Circular Hough Transform using eigenvalues of Covariance Matrix for detecting circles”, 2011 Proceedings of ELMAR, Sep 2011, pp. 397-400. 
[47] http://en.wikipedia.org/wiki/Computational_intelligence.

[48] Shartruhan Modi, Seema Bawa, "Automated Coin Recogtion System using ANN", International Journal of Computer Applications, Jul 2011, vol.26, no.4, pp. 13-18.

[49] T .W. Nattkemper, W. Schubert, T. Hermann, and H. Ritter, "A Hybrid System for Cell Detection in Digital Micrographs”, In Tilg, B. (Ed.), Biomedical Engineering, Proc. BIOMED 2004, ACTA Press.

[50] K. Chattopadhyay, A. Acharya, A. Banerjee, J. Basu and A. Konar, "Fast and Efficient Circle Detection Schemes for Digital Image", Proceedings of First International Conference on Emerging Trends in Engineering and Technology, Jul 2008, pp. 128-133.

[51] Sambarta Dasgupta, Arijit Biswas, Swagatam Das, Ajith Abraham, "Automatic Circle Detection on Images with an Adaptive Bacterial Foraging Algorithm", Proceedings of the 10th annual conference on Genetic and evolutionary computation, Jul 2008, pp. 1695-1696.

[52] Erik Cuevas, Felipe Sención-Echauri, Daniel Zaldivar and Marco Pérez-Cisneros, "Multi-circle detection on images using artificial bee colony (ABC) optimization", Soft Computing, Feb 2012, vol.16, no.2, pp. 281-296.

[53] D. Karaboga, “An Idea Based On Honey Bee Swarm for Numerical Optimization”, Technical Report-TR06, Erciyes University, Engineering Faculty, Computer Engineering Department 2005.

[54] Hiroyuki Kawanishi, Masafumi Hagiwara, "A Shape Detection Method using Improved Genetic Algorithm”, Proceedings of IEEE International Conference on Systems, Man and Cybernetics, Oct 1995, vol.1, pp. 235-240.

[55] Peng-yeng Yin, “A new circle/ellipse detector using genetic algorithms”, Pattern Recognition Letters, Jul 1999, vol.20, no.7, pp. 731-740.

[56] Victor Ayala-Ramirez, Carlos H. Garcia-Capulin, Arturo Perez-Garcia, Raul E. Sanchez-Yanez, "Circle detection on images using genetic algorithms", Pattern Recognition Letters, Apr 2006, vol.27, no.6, pp.652-657.

[57] George Karkavitsas and Maria Rangoussi,"Object Localization in Medical Images Using Genetic Algorithms", International Journal of Signal Processing, 2005, pp.204207.

[58] S. Das, S. Dasgupta, A. Biswas, A. Abraham," Automatic Circle Detection on Images with Annealed Differential Evolution", Proceedings of Eighth International Conference on Hybrid Intelligent Systems, Sep 2008, pp. 684-689. 
[59] G. Roth, M. D. Levine, "Geometric primitive extraction using a genetic algorithm”, IEEE Transactions on Pattern Analysis and Machine Intelligence, Sep 1994, vol.6, no.9, pp.901-905.

[60] Lee A. Segal, Irun R. Cohen, "Design Principles for the Immune System and Other Distributed Autonomous Systems”, Oxford University Press, pp. 12.

[61] Dipankar Dasgupta, Luis Fernando Nino, "Immunological Computation Theory and Applications”, CRC Press, 2009, pp. 1-4.

[62] M. Kleis, T. Hirsch, T. Zseby, "Network Immunity: What can we learn from nature for network protection?”, Proceedings of Bio-Inspired Models of Network, Information and Computing Systems, Dec 2007, pp.283-286.

[63] D. Dasgupta, Z. Ji, F. Gonzalez, "Artificial immune system (AIS) research in the last five years", Proceedings of the 2003 Congress on Evolutionary Computation, Dec 2004, vol.1, pp. 123-130.

[64] N. K. Jerne, “Towards a network theory of the immune system”,. Ann. Immunol. (Inst. Pasteur), 1974, 125C:373-389.

[65] D. Dasgupta, “Advances in artificial immune systems”, IEEE Computational Intelligence Magazine, Nov 2006, vol.1, no.4, pp. 40-49.

[66] D. Dasgupta, N. Attoh-Okine, "Immunity-based systems: a survey", Proceedings of 1997 IEEE International Conference on Systems, Man, and Cybernetics, Oct 1997, vol.1, pp.369-374.

[67] S. Forrest, A. S. Perelson, L. Allen, R. Cherukuri, "Self-Nonself Discrimination in a Computer", Proceedings of IEEE Symposium on Research in Security and Privacy, May 1994, pp. 202-212.

[68] L. N. de Castro, F. J. Von Zuben, "Learning and optimization using the clonal selection principle", IEEE Transactions on Evolutionary Computation, Jun 2002, vol.6, no.3, pp.239-251.

[69] X. Wang, X. Z. Gao, S. J. Ovaska, "Artificial immune optimization methods and applications - a survey", Proceedings of 2004 IEEE International Conference on Systems, Man and Cybernetics, Oct 2004, vol.4, pp. 3415-3420.

[70] D. F. McCoy, V. Devarajan, "Artificial immune systems and aerial image segmentation" , Proceedings of 1997 IEEE International Conference on Systems, Man, and Cybernetics, Oct 1997, vol.1, pp. 867-872.

[71] J. Zhou, D. Dasgupta, Y. Zhiling, T. Hongmei, "Analysis of Dental Images using Artificial Immune Systems”, Proceedings of 2006 IEEE Congress on Evolutionary Computation, 2006, pp. 528-535. 
[72] N. Isa, N. M. Sabri, K. S. Jazahanim, N. K. Taylor, "Application of the Clonal Selection Algorithm in artificial immune systems for shape recognition”, Proceedings of 2010 International Conference on Information Retrieval \& Knowledge Management, Mar 2010, pp. 223-228.

[73] S. Sathyanath, F. Sahin, "An AIS approach to a color image classification problem in a real time industrial application", Proceedings of 2001 IEEE International Conference on Systems, Man, and Cybernetics, 2001, vol.4, pp. 2285-2290.

[74] E. Cuevas, V. Osuna-Enciso, F. Wario, D. Zaldívar, M. Pérez-Cisneros, “Automatic multiple circle detection based on artificial immune systems", Expert Systems with Applications, Jan 2012, vol.29, no.2, pp. 713-722.

[75] http://en.wikipedia.org/wiki/HSL_and_HSV.

[76] Rafael C. Gonzales, Richard E. Woods, "Digital Image Processing Third Edition”, Pearson Prentice Hall, 2008, pp.707-714, 649-650.

[77] Aijun Chen, Guanghui Dong, "Efficient Method for Rapidly Detecting Circles Based on Edge-Tracking”, Proceedings of Second International Symposium on Computational Intelligence and Design, Dec 2009, vol.1, pp. 402-405.

[78] http://en.wikipedia.org/wiki/Precision_and_recall.

[79] Jesse Davis, Mark Goadrich, “The relationship between Precision-Recall and ROC curves", Proceedings of the 23rd international conference on Machine learning, 2006, pp.233-240.

[80] Bin Li, Shancheng Bao, Quan Chen, "The Automated Detection and Scoring Algorithm for Bladder Transitional Cell Carcinoma Screening with PLZ4 Coated Beads”, Medical Physics, 2011, vol.38, issue 6, pp. 3453. 


\section{APPENDIX A: DETECTION STATISTICS}

Table A-1 Manual grading of detection for geometric feature based method

\begin{tabular}{|r|r|r|r|r|r|r|r|}
\hline Pic \# & Image \# & Grade & TruePos & FalsePos & FalseNeg & Precision & Sensitivity \\
\hline 1 & 5065 & 1 & & 0 & 0 & $100 \%$ & $100 \%$ \\
\hline 2 & 5067 & 2 & 4 & 0 & 1 & $100 \%$ & $80 \%$ \\
\hline 3 & 5068 & 2 & 6 & 1 & 1 & $86 \%$ & $86 \%$ \\
\hline 4 & 5069 & 4 & 1 & 0 & 0 & $100 \%$ & $100 \%$ \\
\hline 5 & 5070 & 1 & 1 & 0 & 0 & $100 \%$ & $100 \%$ \\
\hline 6 & 5071 & 4 & 6 & 0 & 0 & $100 \%$ & $100 \%$ \\
\hline 7 & 5072 & 5 & 6 & 1 & 0 & $86 \%$ & $100 \%$ \\
\hline 8 & 5073 & 4 & 5 & 0 & 0 & $100 \%$ & $100 \%$ \\
\hline 9 & 5074 & 5 & 5 & 1 & 0 & $83 \%$ & $100 \%$ \\
\hline 10 & 5076 & 4 & 21 & 5 & 8 & $81 \%$ & $72 \%$ \\
\hline 11 & 5083 & 4 & 15 & 7 & 6 & $68 \%$ & $71 \%$ \\
\hline 12 & 5084 & 4 & 13 & 6 & 7 & $68 \%$ & $65 \%$ \\
\hline 13 & 5085 & 5 & 1 & 0 & 0 & $100 \%$ & $100 \%$ \\
\hline 14 & 5086 & 5 & 1 & 0 & 0 & $100 \%$ & $100 \%$ \\
\hline 15 & 5087 & 5 & 1 & 0 & 0 & $100 \%$ & $100 \%$ \\
\hline 16 & 5088 & 2 & 3 & 0 & 0 & $100 \%$ & $100 \%$ \\
\hline 17 & 5089 & 5 & 9 & 2 & 2 & $82 \%$ & $82 \%$ \\
\hline 18 & 5090 & 4 & 5 & 0 & 0 & $100 \%$ & $100 \%$ \\
\hline 19 & 5091 & 4 & 6 & 0 & 0 & $100 \%$ & $100 \%$ \\
\hline 20 & 5092 & 4 & 6 & 0 & 2 & $100 \%$ & $75 \%$ \\
\hline 21 & 5093 & 4 & 7 & 1 & 0 & $88 \%$ & $100 \%$ \\
\hline 22 & 5094 & 3 & 9 & 1 & 1 & $90 \%$ & $90 \%$ \\
\hline 23 & 5095 & 3 & 18 & 0 & 0 & $100 \%$ & $100 \%$ \\
\hline 24 & 5096 & 4 & 22 & 3 & 0 & $88 \%$ & $100 \%$ \\
\hline 25 & 5097 & 4 & 18 & 0 & 1 & $100 \%$ & $95 \%$ \\
\hline 26 & 5105 & 4 & 10 & 6 & 9 & $63 \%$ & $53 \%$ \\
\hline 27 & 5112 & 5 & 1 & 0 & 0 & $100 \%$ & $100 \%$ \\
\hline 28 & 5114 & 5 & 1 & 0 & 0 & $100 \%$ & $100 \%$ \\
\hline 29 & 5115 & 3 & 2 & 0 & 0 & $100 \%$ & $100 \%$ \\
\hline 30 & 5116 & 5 & 1 & 0 & 0 & $100 \%$ & $100 \%$ \\
\hline 31 & 5117 & 5 & 0 & 1 & 1 & $0 \%$ & $0 \%$ \\
\hline 32 & 5120 & 1 & 18 & 0 & 0 & $100 \%$ & $100 \%$ \\
\hline 33 & 5121 & 1 & 16 & 1 & 0 & $94 \%$ & $100 \%$ \\
\hline 34 & 5122 & 2 & 3 & 0 & 0 & $100 \%$ & $100 \%$ \\
\hline 35 & 5123 & 3 & 5 & 1 & 0 & $83 \%$ & $100 \%$ \\
\hline 36 & 5124 & 3 & 5 & 0 & 0 & $100 \%$ & $100 \%$ \\
\hline 37 & 5127 & 3 & 15 & 2 & 4 & $88 \%$ & $79 \%$ \\
\hline 38 & 5128 & 4 & 13 & 14 & 10 & $48 \%$ & $57 \%$ \\
\hline & & & & & & & \\
\hline
\end{tabular}




\begin{tabular}{|r|r|r|r|r|r|r|r|}
\hline 39 & 5129 & 1 & 11 & 0 & 0 & $100 \%$ & $100 \%$ \\
\hline 40 & 5130 & 1 & 7 & 0 & 0 & $100 \%$ & $100 \%$ \\
\hline 41 & 5131 & 4 & 2 & 1 & 2 & $67 \%$ & $50 \%$ \\
\hline 42 & 5132 & 4 & 3 & 1 & 1 & $75 \%$ & $75 \%$ \\
\hline 43 & 5133 & 1 & 20 & 0 & 0 & $100 \%$ & $100 \%$ \\
\hline 44 & 5134 & 1 & 19 & 0 & 0 & $100 \%$ & $100 \%$ \\
\hline 45 & 5135 & 1 & 21 & 1 & 1 & $95 \%$ & $95 \%$ \\
\hline 46 & 5136 & 1 & 20 & 0 & 0 & $100 \%$ & $100 \%$ \\
\hline 47 & 5137 & 2 & 2 & 0 & 0 & $100 \%$ & $100 \%$ \\
\hline 48 & 5138 & 5 & 2 & 1 & 0 & $67 \%$ & $100 \%$ \\
\hline 49 & 5139 & 5 & 3 & 10 & 12 & $23 \%$ & $20 \%$ \\
\hline 50 & 5140 & 5 & 3 & 3 & 6 & $50 \%$ & $33 \%$ \\
\hline 51 & 5141 & 5 & 5 & 10 & 12 & $33 \%$ & $29 \%$ \\
\hline 52 & 5146 & 5 & 7 & 6 & 3 & $54 \%$ & $70 \%$ \\
\hline 53 & 5147 & 5 & 4 & 4 & 3 & $50 \%$ & $57 \%$ \\
\hline 54 & 5148 & 5 & 12 & 5 & 5 & $71 \%$ & $71 \%$ \\
\hline 55 & 5149 & 1 & 4 & 0 & 0 & $100 \%$ & $100 \%$ \\
\hline 56 & 5150 & 2 & 11 & 0 & 0 & $100 \%$ & $100 \%$ \\
\hline 57 & 5151 & 2 & 11 & 3 & 4 & $79 \%$ & $73 \%$ \\
\hline 58 & 5152 & 3 & 24 & 3 & 6 & $89 \%$ & $80 \%$ \\
\hline 59 & 5153 & 2 & 25 & 5 & 5 & $83 \%$ & $83 \%$ \\
\hline 60 & 5154 & 1 & 23 & 4 & 3 & $85 \%$ & $88 \%$ \\
\hline 61 & 5155 & 1 & 21 & 0 & 0 & $100 \%$ & $100 \%$ \\
\hline 62 & 5156 & 1 & 20 & 0 & 0 & $100 \%$ & $100 \%$ \\
\hline 63 & 5157 & 1 & 11 & 0 & 2 & $100 \%$ & $85 \%$ \\
\hline 64 & 5158 & 1 & 26 & 4 & 6 & $87 \%$ & $81 \%$ \\
\hline 65 & 5159 & 1 & 21 & 0 & 1 & $100 \%$ & $95 \%$ \\
\hline 66 & 5163 & 2 & 19 & 2 & 5 & $90 \%$ & $79 \%$ \\
\hline 67 & 5164 & 1 & 13 & 0 & 3 & $100 \%$ & $81 \%$ \\
\hline 68 & 5165 & 1 & 13 & 0 & 4 & $100 \%$ & $76 \%$ \\
\hline 69 & 5166 & 1 & 11 & 0 & 1 & $100 \%$ & $92 \%$ \\
\hline 70 & 5167 & 1 & 10 & 0 & 1 & $100 \%$ & $91 \%$ \\
\hline 71 & 5168 & 1 & 32 & 0 & 3 & $100 \%$ & $91 \%$ \\
\hline 72 & 5169 & 1 & 31 & 0 & 5 & $100 \%$ & $86 \%$ \\
\hline 73 & 5170 & 1 & 30 & 0 & 0 & $100 \%$ & $100 \%$ \\
\hline 74 & 5171 & 1 & 25 & 0 & 1 & $100 \%$ & $96 \%$ \\
\hline 75 & 5172 & 1 & 26 & 0 & 0 & $100 \%$ & $100 \%$ \\
\hline 76 & 5173 & 1 & 24 & 0 & 4 & $100 \%$ & $86 \%$ \\
\hline 77 & 5174 & 1 & 35 & 0 & 2 & $100 \%$ & $95 \%$ \\
\hline 78 & 5175 & 1 & 16 & 0 & 0 & $100 \%$ & $100 \%$ \\
\hline 79 & 5176 & 1 & 22 & 0 & 4 & $100 \%$ & $85 \%$ \\
\hline 80 & 5177 & 1 & 16 & 0 & 0 & $100 \%$ & $100 \%$ \\
\hline
\end{tabular}




\begin{tabular}{|r|r|r|r|r|r|r|r|}
\hline 81 & 5178 & 1 & 16 & 0 & 0 & $100 \%$ & $100 \%$ \\
\hline 82 & 5179 & 1 & 11 & 0 & 0 & $100 \%$ & $100 \%$ \\
\hline 83 & 5180 & 1 & 9 & 0 & 0 & $100 \%$ & $100 \%$ \\
\hline 84 & 5181 & 1 & 26 & 0 & 0 & $100 \%$ & $100 \%$ \\
\hline 85 & 5182 & 1 & 28 & 0 & 1 & $100 \%$ & $97 \%$ \\
\hline
\end{tabular}

Table A-2 Manual grading of detection for regular Hough Transform

\begin{tabular}{|r|r|r|r|r|r|r|r|}
\hline Pic \# & Image \# & Grade & TruePos & FalsePos & FalseNeg & Precision & Sensitivity \\
\hline 1 & 5065 & 1 & 9 & 0 & 0 & $100 \%$ & $100 \%$ \\
\hline 2 & 5067 & 2 & 3 & 0 & 2 & $100 \%$ & $60 \%$ \\
\hline 3 & 5068 & 2 & 7 & 0 & 1 & $100 \%$ & $88 \%$ \\
\hline 4 & 5069 & 4 & 1 & 0 & 0 & $100 \%$ & $100 \%$ \\
\hline 5 & 5070 & 1 & 1 & 0 & 0 & $100 \%$ & $100 \%$ \\
\hline 6 & 5071 & 4 & 5 & 0 & 0 & $100 \%$ & $100 \%$ \\
\hline 7 & 5072 & 5 & 5 & 0 & 0 & $100 \%$ & $100 \%$ \\
\hline 8 & 5073 & 4 & 5 & 1 & 0 & $83 \%$ & $100 \%$ \\
\hline 9 & 5074 & 5 & 5 & 0 & 0 & $100 \%$ & $100 \%$ \\
\hline 10 & 5076 & 4 & 27 & 4 & 0 & $87 \%$ & $100 \%$ \\
\hline 11 & 5083 & 4 & 16 & 2 & 1 & $89 \%$ & $94 \%$ \\
\hline 12 & 5084 & 4 & 16 & 0 & 1 & $100 \%$ & $94 \%$ \\
\hline 13 & 5085 & 5 & 1 & 0 & 0 & $100 \%$ & $100 \%$ \\
\hline 14 & 5086 & 5 & 1 & 0 & 0 & $100 \%$ & $100 \%$ \\
\hline 15 & 5087 & 5 & 1 & 0 & 0 & $100 \%$ & $100 \%$ \\
\hline 16 & 5088 & 2 & 3 & 0 & 0 & $100 \%$ & $100 \%$ \\
\hline 17 & 5089 & 5 & 13 & 0 & 0 & $100 \%$ & $100 \%$ \\
\hline 18 & 5090 & 4 & 5 & 0 & 0 & $100 \%$ & $100 \%$ \\
\hline 19 & 5091 & 4 & 6 & 0 & 0 & $100 \%$ & $100 \%$ \\
\hline 20 & 5092 & 4 & 8 & 0 & 0 & $100 \%$ & $100 \%$ \\
\hline 21 & 5093 & 4 & 8 & 0 & 0 & $100 \%$ & $100 \%$ \\
\hline 22 & 5094 & 3 & 11 & 0 & 0 & $100 \%$ & $100 \%$ \\
\hline 23 & 5095 & 3 & 17 & 0 & 1 & $100 \%$ & $94 \%$ \\
\hline 24 & 5096 & 4 & 21 & 6 & 1 & $78 \%$ & $95 \%$ \\
\hline 25 & 5097 & 4 & 20 & 3 & 0 & $87 \%$ & $100 \%$ \\
\hline 26 & 5105 & 4 & 17 & 2 & 1 & $89 \%$ & $94 \%$ \\
\hline 27 & 5112 & 5 & 1 & 0 & 0 & $100 \%$ & $100 \%$ \\
\hline 28 & 5114 & 5 & 1 & 3 & 0 & $25 \%$ & $100 \%$ \\
\hline 29 & 5115 & 3 & 2 & 0 & 0 & $100 \%$ & $100 \%$ \\
\hline 30 & 5116 & 5 & 1 & 0 & 0 & $100 \%$ & $100 \%$ \\
\hline 31 & 5117 & 5 & 1 & 0 & 0 & $100 \%$ & $100 \%$ \\
\hline 32 & 5120 & 1 & 12 & 0 & 5 & $100 \%$ & $71 \%$ \\
\hline 33 & 5121 & 1 & 14 & 0 & 2 & $100 \%$ & $88 \%$ \\
\hline
\end{tabular}




\begin{tabular}{|r|r|r|r|r|r|r|r|}
\hline 34 & 5122 & 2 & 3 & 0 & 0 & $100 \%$ & $100 \%$ \\
\hline 35 & 5123 & 3 & 5 & 0 & 0 & $100 \%$ & $100 \%$ \\
\hline 36 & 5124 & 3 & 5 & 0 & 0 & $100 \%$ & $100 \%$ \\
\hline 37 & 5127 & 3 & 18 & 4 & 0 & $82 \%$ & $100 \%$ \\
\hline 38 & 5128 & 4 & 25 & 4 & 0 & $86 \%$ & $100 \%$ \\
\hline 39 & 5129 & 1 & 7 & 0 & 3 & $100 \%$ & $70 \%$ \\
\hline 40 & 5130 & 1 & 5 & 0 & 3 & $100 \%$ & $63 \%$ \\
\hline 41 & 5131 & 4 & 4 & 1 & 0 & $80 \%$ & $100 \%$ \\
\hline 42 & 5132 & 4 & 4 & 1 & 0 & $80 \%$ & $100 \%$ \\
\hline 43 & 5133 & 1 & 13 & 0 & 5 & $100 \%$ & $72 \%$ \\
\hline 44 & 5134 & 1 & 16 & 0 & 3 & $100 \%$ & $84 \%$ \\
\hline 45 & 5135 & 1 & 14 & 0 & 5 & $100 \%$ & $74 \%$ \\
\hline 46 & 5136 & 1 & 20 & 0 & 1 & $100 \%$ & $95 \%$ \\
\hline 47 & 5137 & 2 & 2 & 0 & 0 & $100 \%$ & $100 \%$ \\
\hline 48 & 5138 & 5 & 4 & 0 & 0 & $100 \%$ & $100 \%$ \\
\hline 49 & 5139 & 5 & 14 & 15 & 0 & $48 \%$ & $100 \%$ \\
\hline 50 & 5140 & 5 & 11 & 6 & 0 & $65 \%$ & $100 \%$ \\
\hline 51 & 5141 & 5 & 17 & 10 & 1 & $63 \%$ & $94 \%$ \\
\hline 52 & 5146 & 5 & 9 & 3 & 1 & $75 \%$ & $90 \%$ \\
\hline 53 & 5147 & 5 & 7 & 3 & 0 & $70 \%$ & $100 \%$ \\
\hline 54 & 5148 & 5 & 16 & 9 & 0 & $64 \%$ & $100 \%$ \\
\hline 55 & 5149 & 1 & 5 & 0 & 0 & $100 \%$ & $100 \%$ \\
\hline 56 & 5150 & 2 & 10 & 1 & 1 & $91 \%$ & $91 \%$ \\
\hline 57 & 5151 & 2 & 14 & 0 & 0 & $100 \%$ & $100 \%$ \\
\hline 58 & 5152 & 3 & 28 & 0 & 2 & $100 \%$ & $93 \%$ \\
\hline 59 & 5153 & 2 & 30 & 0 & 0 & $100 \%$ & $100 \%$ \\
\hline 60 & 5154 & 1 & 24 & 1 & 3 & $96 \%$ & $89 \%$ \\
\hline 61 & 5155 & 1 & 21 & 0 & 0 & $100 \%$ & $100 \%$ \\
\hline 62 & 5156 & 1 & 21 & 0 & 0 & $100 \%$ & $100 \%$ \\
\hline 63 & 5157 & 1 & 11 & 0 & 2 & $100 \%$ & $85 \%$ \\
\hline 64 & 5158 & 1 & 29 & 0 & 2 & $100 \%$ & $94 \%$ \\
\hline 65 & 5159 & 1 & 21 & 0 & 1 & $100 \%$ & $95 \%$ \\
\hline 66 & 5163 & 2 & 25 & 1 & 1 & $96 \%$ & $96 \%$ \\
\hline 67 & 5164 & 1 & 15 & 0 & 3 & $100 \%$ & $83 \%$ \\
\hline 68 & 5165 & 1 & 14 & 0 & 3 & $100 \%$ & $82 \%$ \\
\hline 69 & 5166 & 1 & 11 & 0 & 1 & $100 \%$ & $92 \%$ \\
\hline 70 & 5167 & 1 & 12 & 0 & 0 & $100 \%$ & $100 \%$ \\
\hline 71 & 5168 & 1 & 29 & 0 & 6 & $100 \%$ & $83 \%$ \\
\hline 72 & 5169 & 1 & 36 & 0 & 1 & $100 \%$ & $97 \%$ \\
\hline 73 & 5170 & 1 & 27 & 0 & 2 & $100 \%$ & $93 \%$ \\
\hline 74 & 5171 & 1 & 23 & 0 & 2 & $100 \%$ & $92 \%$ \\
\hline 75 & 5172 & 1 & 22 & 0 & 2 & $100 \%$ & $92 \%$ \\
\hline
\end{tabular}




\begin{tabular}{|r|r|r|r|r|r|r|r|}
\hline 76 & 5173 & 1 & 24 & 0 & 3 & $100 \%$ & $89 \%$ \\
\hline 77 & 5174 & 1 & 34 & 2 & 3 & $94 \%$ & $92 \%$ \\
\hline 78 & 5175 & 1 & 9 & 0 & 7 & $100 \%$ & $56 \%$ \\
\hline 79 & 5176 & 1 & 20 & 0 & 6 & $100 \%$ & $77 \%$ \\
\hline 80 & 5177 & 1 & 11 & 0 & 5 & $100 \%$ & $69 \%$ \\
\hline 81 & 5178 & 1 & 13 & 0 & 3 & $100 \%$ & $81 \%$ \\
\hline 82 & 5179 & 1 & 10 & 0 & 1 & $100 \%$ & $91 \%$ \\
\hline 83 & 5180 & 1 & 9 & 0 & 0 & $100 \%$ & $100 \%$ \\
\hline 84 & 5181 & 1 & 23 & 0 & 4 & $100 \%$ & $85 \%$ \\
\hline 85 & 5182 & 1 & 26 & 0 & 2 & $100 \%$ & $93 \%$ \\
\hline
\end{tabular}

Table A-3 Manual grading of detection for Modified Hough Transform

\begin{tabular}{|c|c|c|c|c|c|c|c|}
\hline Pic \# & Image \# & Grade & TruePos & FalsePos & FalseNeg & Precision & Sensitivity \\
\hline 1 & 5065 & 1 & 9 & 0 & 0 & $100 \%$ & $100 \%$ \\
\hline 2 & 5067 & 2 & 5 & 0 & 0 & $100 \%$ & $100 \%$ \\
\hline 3 & 5068 & 2 & 6 & 0 & 2 & $100 \%$ & $75 \%$ \\
\hline 4 & 5069 & 4 & 1 & 0 & 0 & $100 \%$ & $100 \%$ \\
\hline 5 & 5070 & 1 & 1 & 0 & 0 & $100 \%$ & $100 \%$ \\
\hline 6 & 5071 & 4 & 6 & 0 & 0 & $100 \%$ & $100 \%$ \\
\hline 7 & 5072 & 5 & 6 & 0 & 0 & $100 \%$ & $100 \%$ \\
\hline 8 & 5073 & 4 & 5 & 0 & 0 & $100 \%$ & $100 \%$ \\
\hline 9 & 5074 & 5 & 4 & 0 & 1 & $100 \%$ & $80 \%$ \\
\hline 10 & 5076 & 4 & 28 & 1 & 2 & $97 \%$ & $93 \%$ \\
\hline 11 & 5083 & 4 & 18 & 1 & 0 & $95 \%$ & $100 \%$ \\
\hline 12 & 5084 & 4 & 18 & 0 & 0 & $100 \%$ & $100 \%$ \\
\hline 13 & 5085 & 5 & 1 & 0 & 0 & $100 \%$ & $100 \%$ \\
\hline 14 & 5086 & 5 & 1 & 0 & 0 & $100 \%$ & $100 \%$ \\
\hline 15 & 5087 & 5 & 1 & 0 & 0 & $100 \%$ & $100 \%$ \\
\hline 16 & 5088 & 2 & 3 & 0 & 0 & $100 \%$ & $100 \%$ \\
\hline 17 & 5089 & 5 & 11 & 1 & 1 & $92 \%$ & $92 \%$ \\
\hline 18 & 5090 & 4 & 5 & 0 & 0 & $100 \%$ & $100 \%$ \\
\hline 19 & 5091 & 4 & 6 & 0 & 0 & $100 \%$ & $100 \%$ \\
\hline 20 & 5092 & 4 & 7 & 0 & 1 & $100 \%$ & $88 \%$ \\
\hline 21 & 5093 & 4 & 8 & 0 & 0 & $100 \%$ & $100 \%$ \\
\hline 22 & 5094 & 3 & 11 & 0 & 0 & $100 \%$ & $100 \%$ \\
\hline 23 & 5095 & 3 & 19 & 0 & 0 & $100 \%$ & $100 \%$ \\
\hline 24 & 5096 & 4 & 23 & 2 & 0 & $92 \%$ & $100 \%$ \\
\hline 25 & 5097 & 4 & 20 & 2 & 0 & $91 \%$ & $100 \%$ \\
\hline 26 & 5105 & 4 & 15 & 0 & 4 & $100 \%$ & $79 \%$ \\
\hline 27 & 5112 & 5 & 1 & 0 & 0 & $100 \%$ & $100 \%$ \\
\hline 28 & 5114 & 5 & 1 & 0 & 0 & $100 \%$ & $100 \%$ \\
\hline
\end{tabular}




\begin{tabular}{|r|r|r|r|r|r|r|r|}
\hline 29 & 5115 & 3 & 2 & 0 & 0 & $100 \%$ & $100 \%$ \\
\hline 30 & 5116 & 5 & 1 & 0 & 0 & $100 \%$ & $100 \%$ \\
\hline 31 & 5117 & 5 & 1 & 0 & 0 & $100 \%$ & $100 \%$ \\
\hline 32 & 5120 & 1 & 18 & 0 & 0 & $100 \%$ & $100 \%$ \\
\hline 33 & 5121 & 1 & 17 & 0 & 0 & $100 \%$ & $100 \%$ \\
\hline 34 & 5122 & 2 & 3 & 0 & 0 & $100 \%$ & $100 \%$ \\
\hline 35 & 5123 & 3 & 5 & 0 & 0 & $100 \%$ & $100 \%$ \\
\hline 36 & 5124 & 3 & 5 & 0 & 0 & $100 \%$ & $100 \%$ \\
\hline 37 & 5127 & 3 & 17 & 1 & 1 & $94 \%$ & $94 \%$ \\
\hline 38 & 5128 & 4 & 21 & 1 & 3 & $95 \%$ & $88 \%$ \\
\hline 39 & 5129 & 1 & 10 & 0 & 0 & $100 \%$ & $100 \%$ \\
\hline 40 & 5130 & 1 & 8 & 0 & 0 & $100 \%$ & $100 \%$ \\
\hline 41 & 5131 & 4 & 3 & 0 & 0 & $100 \%$ & $100 \%$ \\
\hline 42 & 5132 & 4 & 4 & 0 & 0 & $100 \%$ & $100 \%$ \\
\hline 43 & 5133 & 1 & 18 & 0 & 0 & $100 \%$ & $100 \%$ \\
\hline 44 & 5134 & 1 & 19 & 1 & 0 & $95 \%$ & $100 \%$ \\
\hline 45 & 5135 & 1 & 21 & 0 & 0 & $100 \%$ & $100 \%$ \\
\hline 46 & 5136 & 1 & 21 & 0 & 0 & $100 \%$ & $100 \%$ \\
\hline 47 & 5137 & 2 & 2 & 0 & 0 & $100 \%$ & $100 \%$ \\
\hline 48 & 5138 & 5 & 4 & 0 & 0 & $100 \%$ & $100 \%$ \\
\hline 49 & 5139 & 5 & 7 & 0 & 7 & $100 \%$ & $50 \%$ \\
\hline 50 & 5140 & 5 & 9 & 1 & 1 & $90 \%$ & $90 \%$ \\
\hline 51 & 5141 & 5 & 14 & 0 & 3 & $100 \%$ & $82 \%$ \\
\hline 52 & 5146 & 5 & 9 & 0 & 1 & $100 \%$ & $90 \%$ \\
\hline 53 & 5147 & 5 & 7 & 1 & 0 & $88 \%$ & $100 \%$ \\
\hline 54 & 5148 & 5 & 15 & 0 & 2 & $100 \%$ & $88 \%$ \\
\hline 55 & 5149 & 1 & 4 & 0 & 0 & $100 \%$ & $100 \%$ \\
\hline 56 & 5150 & 2 & 11 & 0 & 0 & $100 \%$ & $100 \%$ \\
\hline 57 & 5151 & 2 & 13 & 0 & 0 & $100 \%$ & $100 \%$ \\
\hline 58 & 5152 & 3 & 27 & 0 & 3 & $100 \%$ & $90 \%$ \\
\hline 59 & 5153 & 2 & 31 & 0 & 1 & $100 \%$ & $97 \%$ \\
\hline 60 & 5154 & 1 & 26 & 1 & 0 & $96 \%$ & $100 \%$ \\
\hline 61 & 5155 & 1 & 20 & 0 & 1 & $100 \%$ & $95 \%$ \\
\hline 62 & 5156 & 1 & 22 & 0 & 0 & $100 \%$ & $100 \%$ \\
\hline 63 & 5157 & 1 & 12 & 0 & 2 & $100 \%$ & $86 \%$ \\
\hline 64 & 5158 & 1 & 32 & 0 & 0 & $100 \%$ & $100 \%$ \\
\hline 65 & 5159 & 1 & 22 & 0 & 0 & $100 \%$ & $100 \%$ \\
\hline 66 & 5163 & 2 & 26 & 0 & 0 & $100 \%$ & $100 \%$ \\
\hline 67 & 5164 & 1 & 16 & 0 & 0 & $100 \%$ & $100 \%$ \\
\hline 68 & 5165 & 1 & 17 & 0 & 0 & $100 \%$ & $100 \%$ \\
\hline 69 & 5166 & 1 & 12 & 0 & 0 & $100 \%$ & $100 \%$ \\
\hline 70 & 5167 & 1 & 11 & 0 & 0 & $100 \%$ & $100 \%$ \\
\hline
\end{tabular}




\begin{tabular}{|r|r|r|r|r|r|r|r|}
\hline 71 & 5168 & 1 & 36 & 1 & 0 & $97 \%$ & $100 \%$ \\
\hline 72 & 5169 & 1 & 40 & 0 & 0 & $100 \%$ & $100 \%$ \\
\hline 73 & 5170 & 1 & 29 & 0 & 0 & $100 \%$ & $100 \%$ \\
\hline 74 & 5171 & 1 & 26 & 0 & 0 & $100 \%$ & $100 \%$ \\
\hline 75 & 5172 & 1 & 26 & 0 & 0 & $100 \%$ & $100 \%$ \\
\hline 76 & 5173 & 1 & 29 & 0 & 0 & $100 \%$ & $100 \%$ \\
\hline 77 & 5174 & 1 & 38 & 0 & 0 & $100 \%$ & $100 \%$ \\
\hline 78 & 5175 & 1 & 16 & 0 & 0 & $100 \%$ & $100 \%$ \\
\hline 79 & 5176 & 1 & 27 & 0 & 0 & $100 \%$ & $100 \%$ \\
\hline 80 & 5177 & 1 & 16 & 0 & 0 & $100 \%$ & $100 \%$ \\
\hline 81 & 5178 & 1 & 17 & 0 & 0 & $100 \%$ & $100 \%$ \\
\hline 82 & 5179 & 1 & 11 & 0 & 0 & $100 \%$ & $100 \%$ \\
\hline 83 & 5180 & 1 & 9 & 0 & 0 & $100 \%$ & $100 \%$ \\
\hline 84 & 5181 & 1 & 28 & 0 & 0 & $100 \%$ & $100 \%$ \\
\hline 85 & 5182 & 1 & 27 & 0 & 1 & $100 \%$ & $96 \%$ \\
\hline
\end{tabular}

Table A-4 Manual grading of detection for Artificial Immune System

\begin{tabular}{|r|r|r|r|r|r|r|r|}
\hline Pic \# & Image \# & Grade & TruePos & FalsePos & FalseNeg & Precision & Sensitivity \\
\hline 1 & 5065 & 1 & 9 & 0 & 0 & $100 \%$ & $100 \%$ \\
\hline 2 & 5067 & 2 & 6 & 0 & 0 & $100 \%$ & $100 \%$ \\
\hline 3 & 5068 & 2 & 9 & 0 & 0 & $100 \%$ & $100 \%$ \\
\hline 4 & 5069 & 4 & 1 & 0 & 0 & $100 \%$ & $100 \%$ \\
\hline 5 & 5070 & 1 & 1 & 0 & 0 & $100 \%$ & $100 \%$ \\
\hline 6 & 5071 & 4 & 6 & 0 & 0 & $100 \%$ & $100 \%$ \\
\hline 7 & 5072 & 5 & 6 & 0 & 0 & $100 \%$ & $100 \%$ \\
\hline 8 & 5073 & 4 & 8 & 0 & 0 & $100 \%$ & $100 \%$ \\
\hline 9 & 5074 & 5 & 5 & 0 & 0 & $100 \%$ & $100 \%$ \\
\hline 10 & 5076 & 4 & 34 & 3 & 2 & $92 \%$ & $94 \%$ \\
\hline 11 & 5083 & 4 & 22 & 1 & 2 & $96 \%$ & $92 \%$ \\
\hline 12 & 5084 & 4 & 21 & 2 & 1 & $91 \%$ & $95 \%$ \\
\hline 13 & 5085 & 5 & 1 & 0 & 0 & $100 \%$ & $100 \%$ \\
\hline 14 & 5086 & 5 & 1 & 0 & 0 & $100 \%$ & $100 \%$ \\
\hline 15 & 5087 & 5 & 1 & 0 & 0 & $100 \%$ & $100 \%$ \\
\hline 16 & 5088 & 2 & 3 & 0 & 0 & $100 \%$ & $100 \%$ \\
\hline 17 & 5089 & 5 & 18 & 1 & 0 & $95 \%$ & $100 \%$ \\
\hline 18 & 5090 & 4 & 5 & 0 & 0 & $100 \%$ & $100 \%$ \\
\hline 19 & 5091 & 4 & 9 & 0 & 0 & $100 \%$ & $100 \%$ \\
\hline 20 & 5092 & 4 & 10 & 0 & 0 & $100 \%$ & $100 \%$ \\
\hline 21 & 5093 & 4 & 9 & 1 & 1 & $90 \%$ & $90 \%$ \\
\hline 22 & 5094 & 3 & 14 & 0 & 0 & $100 \%$ & $100 \%$ \\
\hline 23 & 5095 & 3 & 26 & 2 & 0 & $93 \%$ & $100 \%$ \\
\hline
\end{tabular}




\begin{tabular}{|r|r|r|r|r|r|r|r|}
\hline 24 & 5096 & 4 & 29 & 2 & 0 & $94 \%$ & $100 \%$ \\
\hline 25 & 5097 & 4 & 23 & 1 & 0 & $96 \%$ & $100 \%$ \\
\hline 26 & 5105 & 4 & 23 & 2 & 0 & $92 \%$ & $100 \%$ \\
\hline 27 & 5112 & 5 & 1 & 0 & 0 & $100 \%$ & $100 \%$ \\
\hline 28 & 5114 & 5 & 1 & 0 & 0 & $100 \%$ & $100 \%$ \\
\hline 29 & 5115 & 3 & 2 & 0 & 0 & $100 \%$ & $100 \%$ \\
\hline 30 & 5116 & 5 & 1 & 0 & 0 & $100 \%$ & $100 \%$ \\
\hline 31 & 5117 & 5 & 1 & 0 & 0 & $100 \%$ & $100 \%$ \\
\hline 32 & 5120 & 1 & 19 & 0 & 0 & $100 \%$ & $100 \%$ \\
\hline 33 & 5121 & 1 & 21 & 1 & 0 & $95 \%$ & $100 \%$ \\
\hline 34 & 5122 & 2 & 3 & 0 & 0 & $100 \%$ & $100 \%$ \\
\hline 35 & 5123 & 3 & 5 & 0 & 0 & $100 \%$ & $100 \%$ \\
\hline 36 & 5124 & 3 & 5 & 0 & 0 & $100 \%$ & $100 \%$ \\
\hline 37 & 5127 & 3 & 20 & 2 & 2 & $91 \%$ & $91 \%$ \\
\hline 38 & 5128 & 4 & 29 & 1 & 2 & $97 \%$ & $94 \%$ \\
\hline 39 & 5129 & 1 & 12 & 0 & 0 & $100 \%$ & $100 \%$ \\
\hline 40 & 5130 & 1 & 8 & 0 & 0 & $100 \%$ & $100 \%$ \\
\hline 41 & 5131 & 4 & 4 & 0 & 0 & $100 \%$ & $100 \%$ \\
\hline 42 & 5132 & 4 & 3 & 0 & 1 & $100 \%$ & $75 \%$ \\
\hline 43 & 5133 & 1 & 25 & 1 & 0 & $96 \%$ & $100 \%$ \\
\hline 44 & 5134 & 1 & 24 & 0 & 0 & $100 \%$ & $100 \%$ \\
\hline 45 & 5135 & 1 & 26 & 0 & 0 & $100 \%$ & $100 \%$ \\
\hline 46 & 5136 & 1 & 24 & 1 & 0 & $96 \%$ & $100 \%$ \\
\hline 47 & 5137 & 2 & 2 & 0 & 0 & $100 \%$ & $100 \%$ \\
\hline 48 & 5138 & 5 & 4 & 0 & 0 & $100 \%$ & $100 \%$ \\
\hline 49 & 5139 & 5 & 11 & 3 & 4 & $79 \%$ & $73 \%$ \\
\hline 50 & 5140 & 5 & 11 & 1 & 0 & $92 \%$ & $100 \%$ \\
\hline 51 & 5141 & 5 & 20 & 1 & 1 & $95 \%$ & $95 \%$ \\
\hline 52 & 5146 & 5 & 10 & 0 & 0 & $100 \%$ & $100 \%$ \\
\hline 53 & 5147 & 5 & 7 & 0 & 0 & $100 \%$ & $100 \%$ \\
\hline 54 & 5148 & 5 & 18 & 3 & 1 & $86 \%$ & $95 \%$ \\
\hline 55 & 5149 & 1 & 5 & 0 & 0 & $100 \%$ & $100 \%$ \\
\hline 56 & 5150 & 2 & 12 & 0 & 0 & $100 \%$ & $100 \%$ \\
\hline 57 & 5151 & 2 & 20 & 1 & 1 & $95 \%$ & $95 \%$ \\
\hline 58 & 5152 & 3 & 38 & 2 & 1 & $95 \%$ & $97 \%$ \\
\hline 59 & 5153 & 2 & 37 & 2 & 2 & $95 \%$ & $95 \%$ \\
\hline 60 & 5154 & 1 & 36 & 2 & 2 & $95 \%$ & $95 \%$ \\
\hline 61 & 5155 & 1 & 27 & 1 & 0 & $96 \%$ & $100 \%$ \\
\hline 62 & 5156 & 1 & 30 & 2 & 0 & $94 \%$ & $100 \%$ \\
\hline 63 & 5157 & 1 & 21 & 0 & 0 & $100 \%$ & $100 \%$ \\
\hline 64 & 5158 & 1 & 42 & 4 & 0 & $91 \%$ & $100 \%$ \\
\hline 65 & 5159 & 1 & 29 & 2 & 0 & $94 \%$ & $100 \%$ \\
\hline
\end{tabular}




\begin{tabular}{|r|r|r|r|r|r|r|r|}
\hline 66 & 5163 & 2 & 30 & 3 & 0 & $91 \%$ & $100 \%$ \\
\hline 67 & 5164 & 1 & 16 & 0 & 0 & $100 \%$ & $100 \%$ \\
\hline 68 & 5165 & 1 & 21 & 1 & 0 & $95 \%$ & $100 \%$ \\
\hline 69 & 5166 & 1 & 13 & 0 & 0 & $100 \%$ & $100 \%$ \\
\hline 70 & 5167 & 1 & 14 & 0 & 0 & $100 \%$ & $100 \%$ \\
\hline 71 & 5168 & 1 & 45 & 3 & 0 & $94 \%$ & $100 \%$ \\
\hline 72 & 5169 & 1 & 46 & 4 & 5 & $92 \%$ & $90 \%$ \\
\hline 73 & 5170 & 1 & 37 & 1 & 0 & $97 \%$ & $100 \%$ \\
\hline 74 & 5171 & 1 & 31 & 1 & 0 & $97 \%$ & $100 \%$ \\
\hline 75 & 5172 & 1 & 32 & 0 & 0 & $100 \%$ & $100 \%$ \\
\hline 76 & 5173 & 1 & 33 & 1 & 0 & $97 \%$ & $100 \%$ \\
\hline 77 & 5174 & 1 & 49 & 2 & 0 & $96 \%$ & $100 \%$ \\
\hline 78 & 5175 & 1 & 21 & 0 & 0 & $100 \%$ & $100 \%$ \\
\hline 79 & 5176 & 1 & 31 & 0 & 0 & $100 \%$ & $100 \%$ \\
\hline 80 & 5177 & 1 & 21 & 0 & 0 & $100 \%$ & $100 \%$ \\
\hline 81 & 5178 & 1 & 17 & 1 & 4 & $94 \%$ & $81 \%$ \\
\hline 82 & 5179 & 1 & 13 & 0 & 0 & $100 \%$ & $100 \%$ \\
\hline 83 & 5180 & 1 & 9 & 0 & 0 & $100 \%$ & $100 \%$ \\
\hline 84 & 5181 & 1 & 31 & 0 & 0 & $100 \%$ & $100 \%$ \\
\hline 85 & 5182 & 1 & 35 & 0 & 0 & $100 \%$ & $100 \%$ \\
\hline
\end{tabular}




\section{APPENDIX B: MATLAB SOURCE CODE}

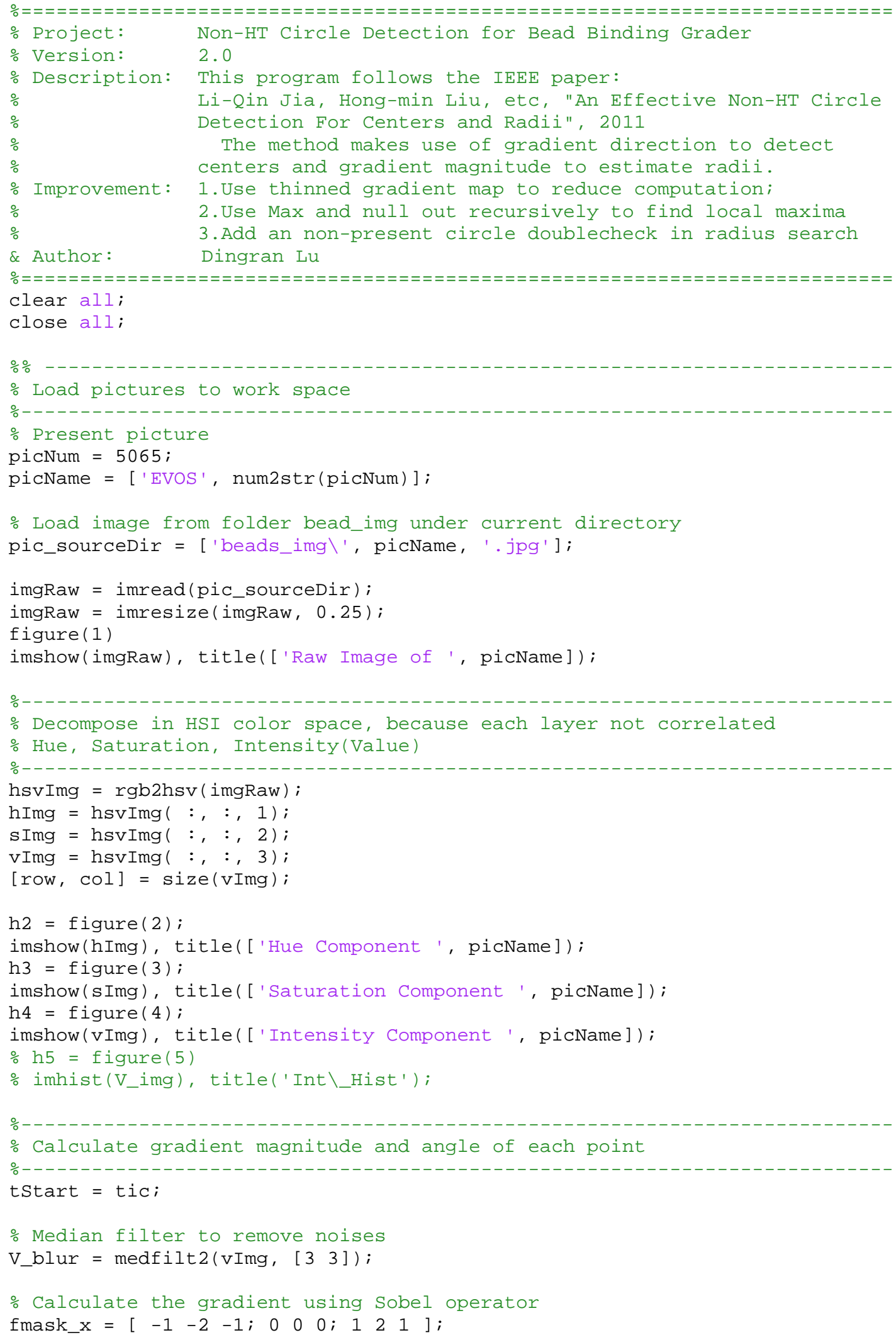




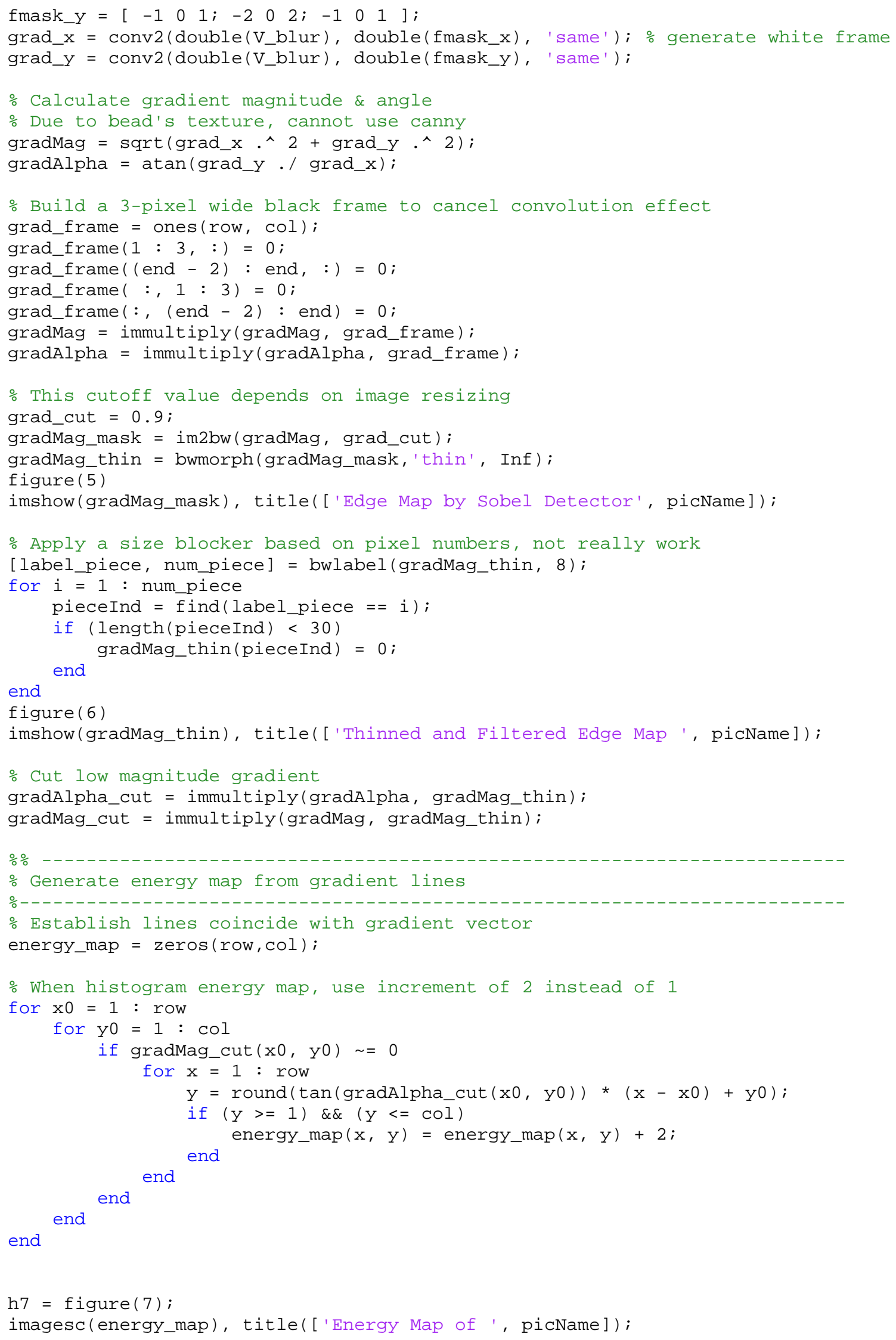




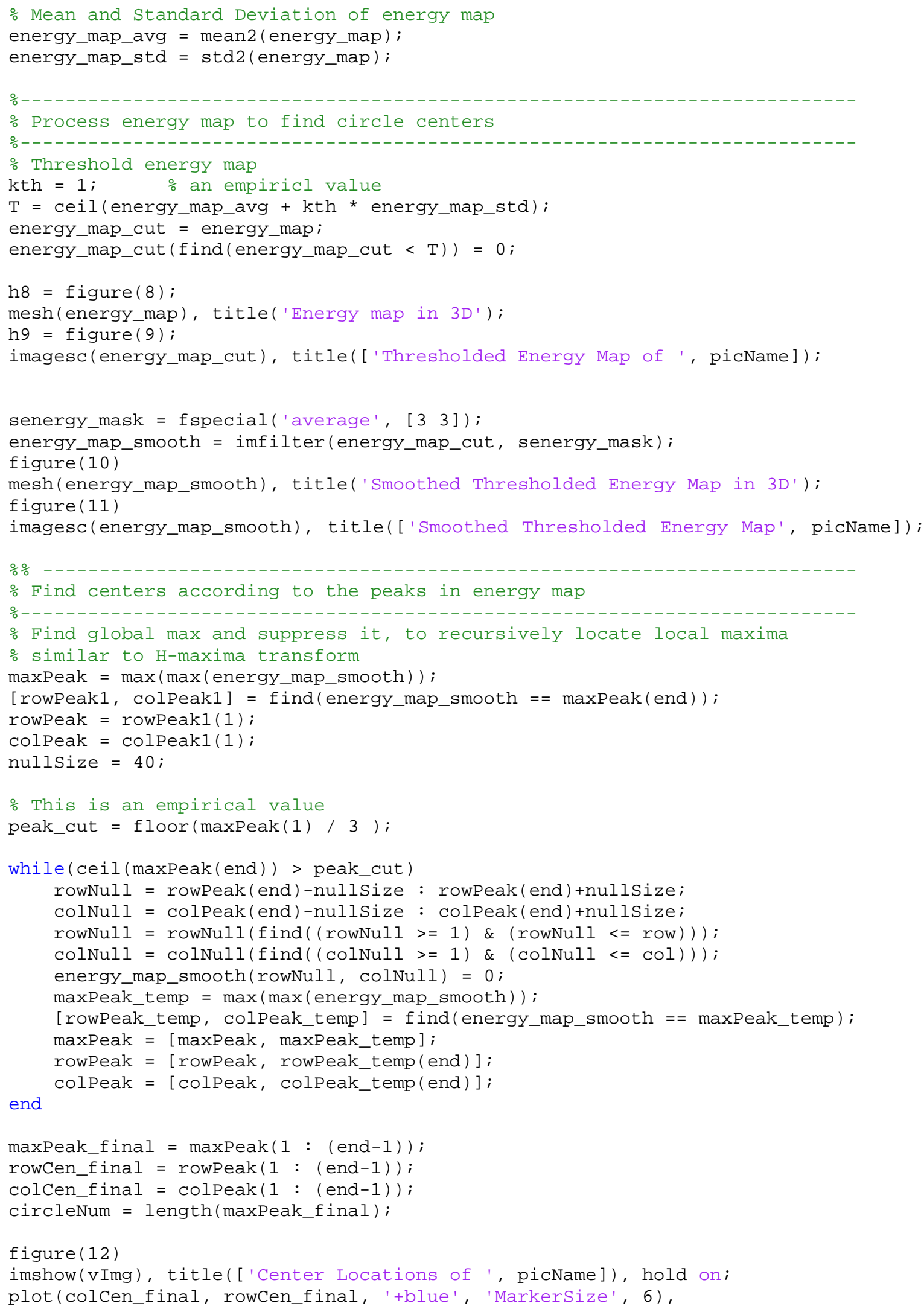




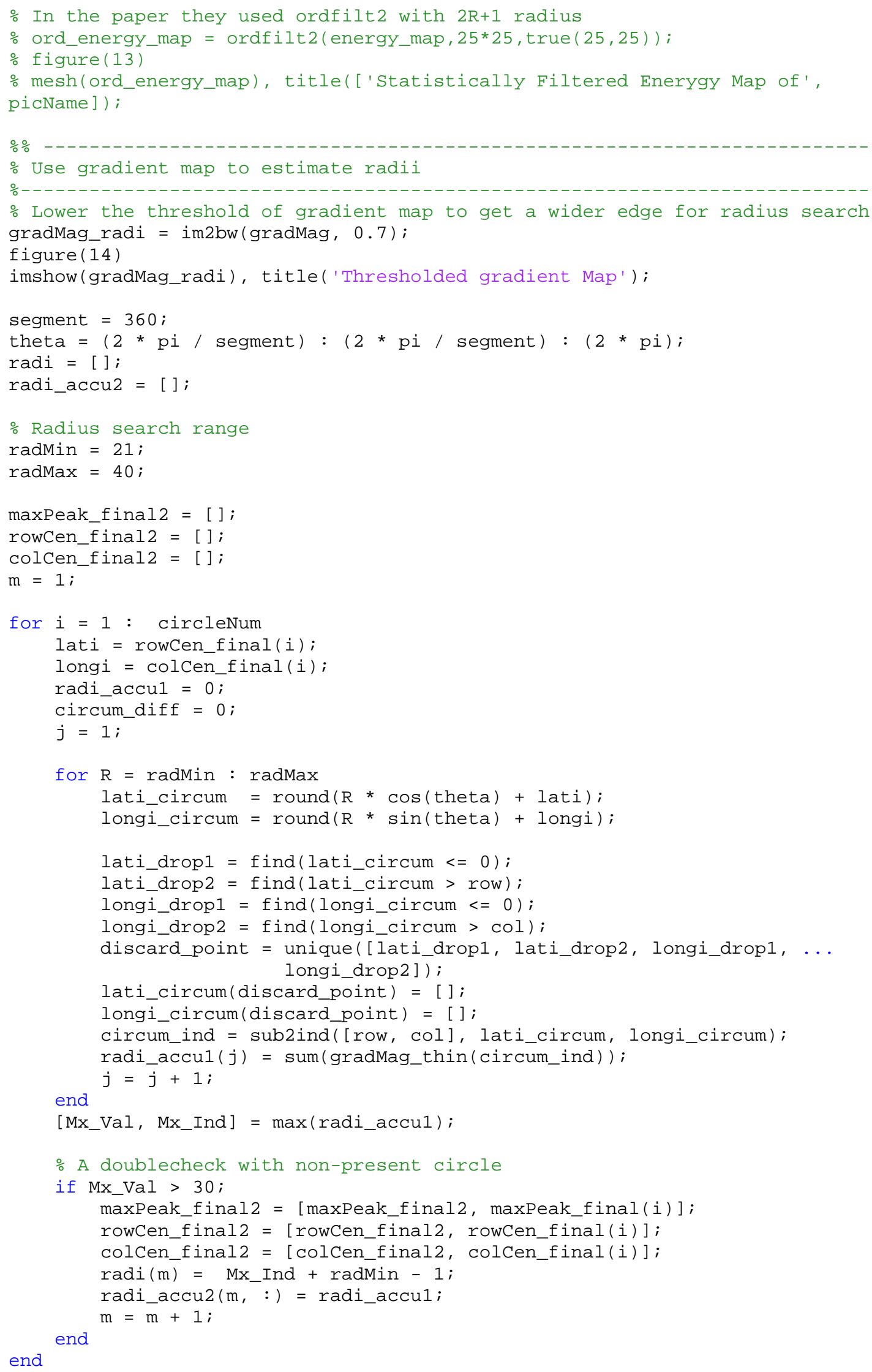




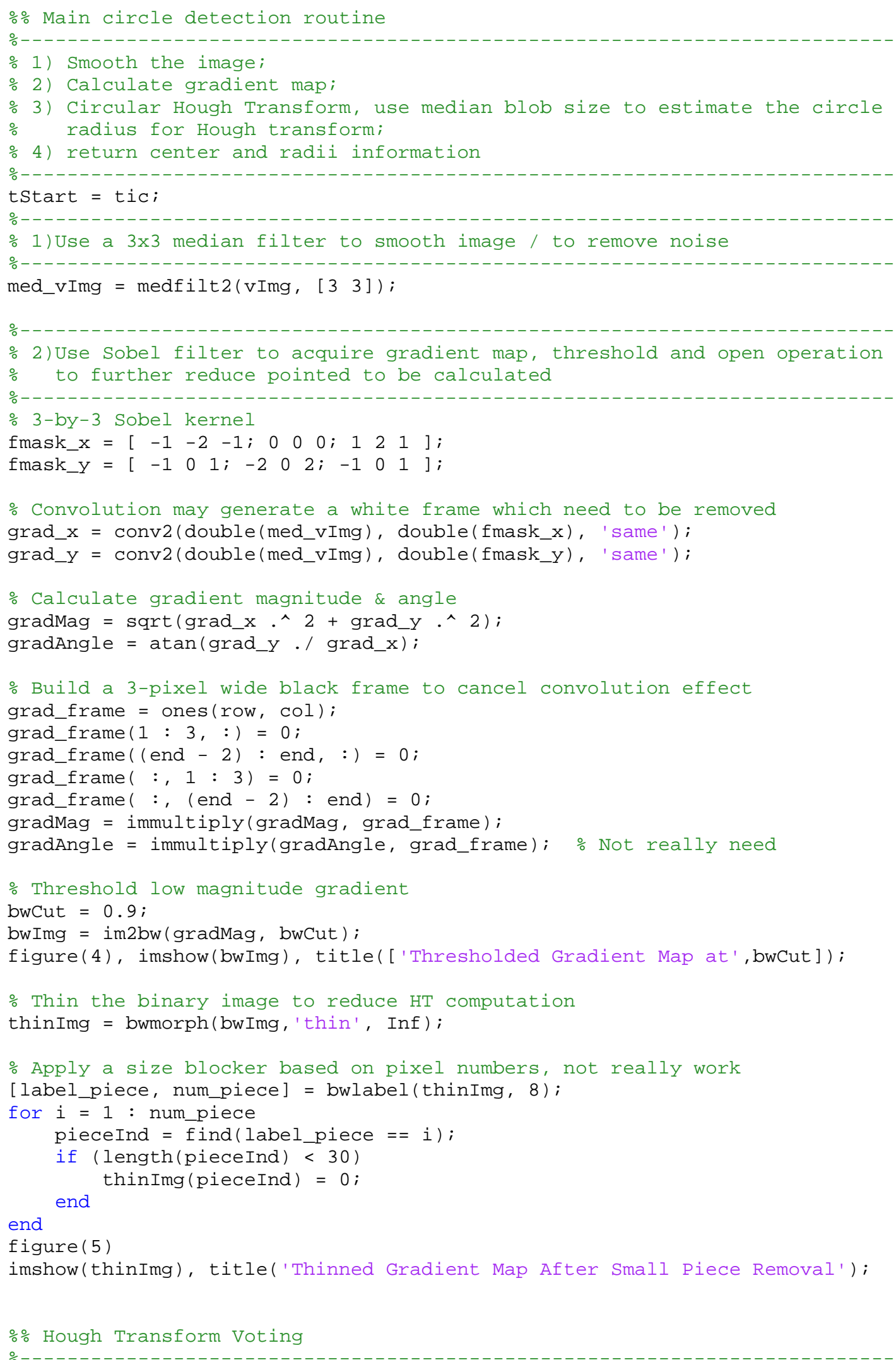




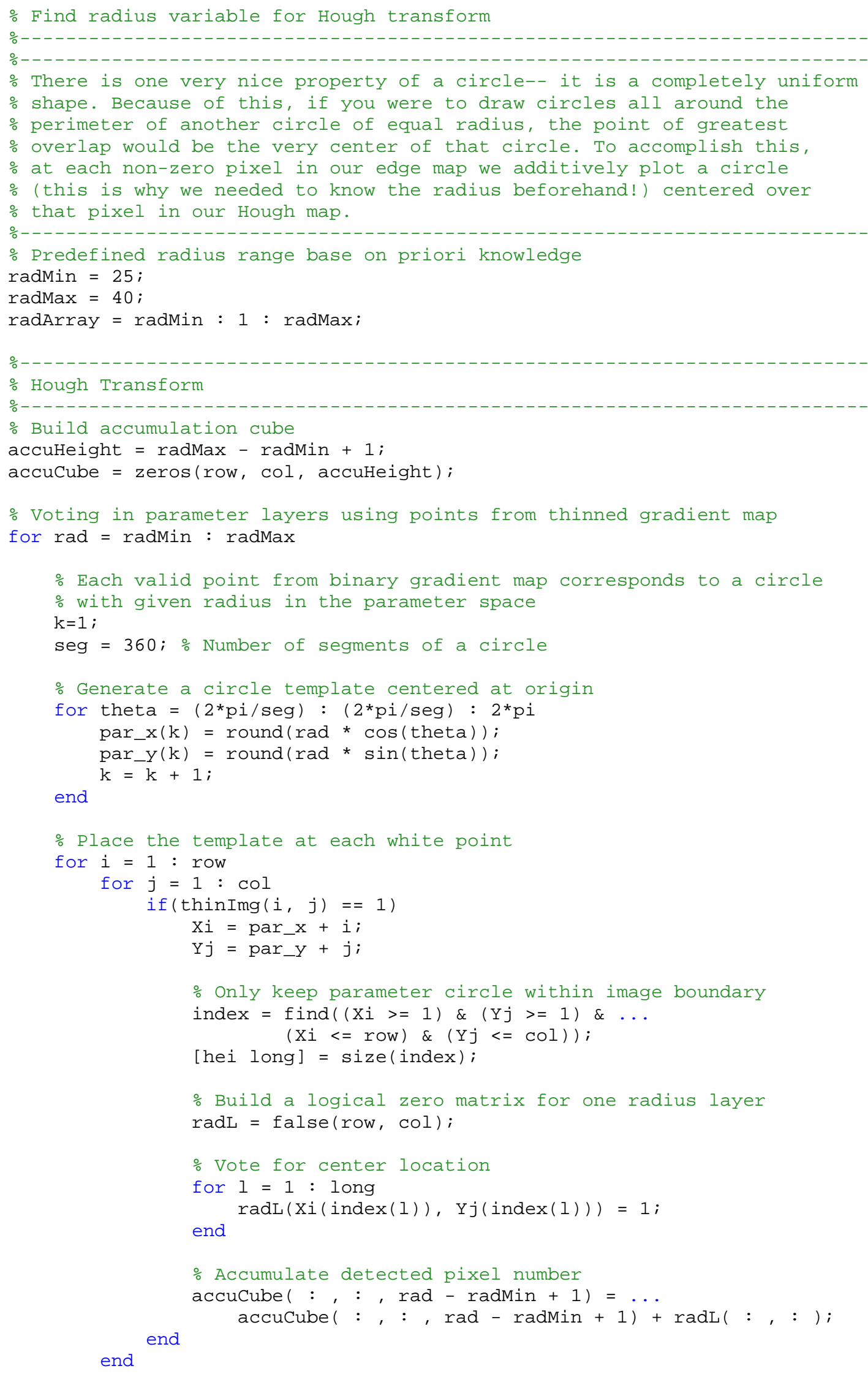




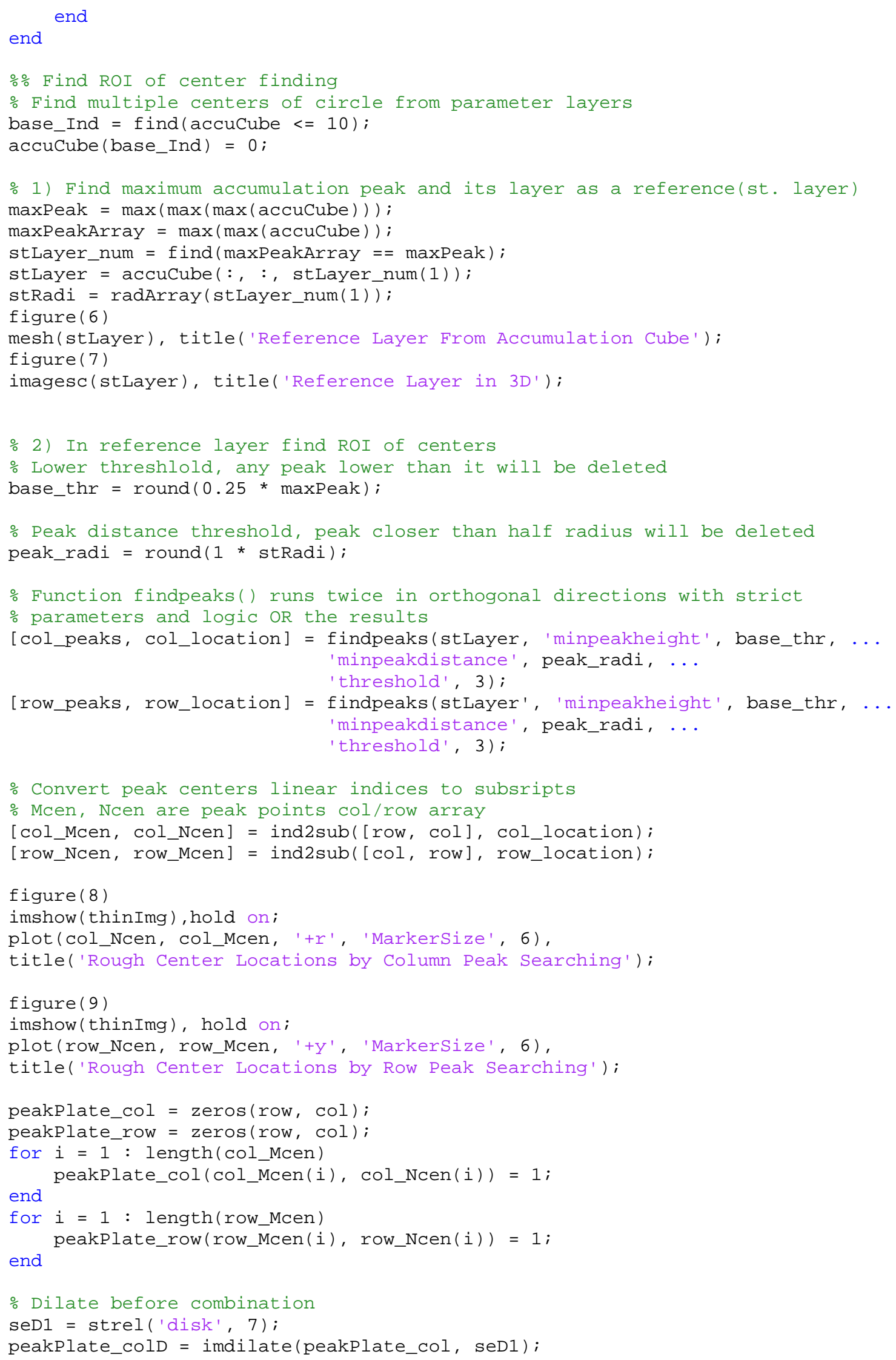




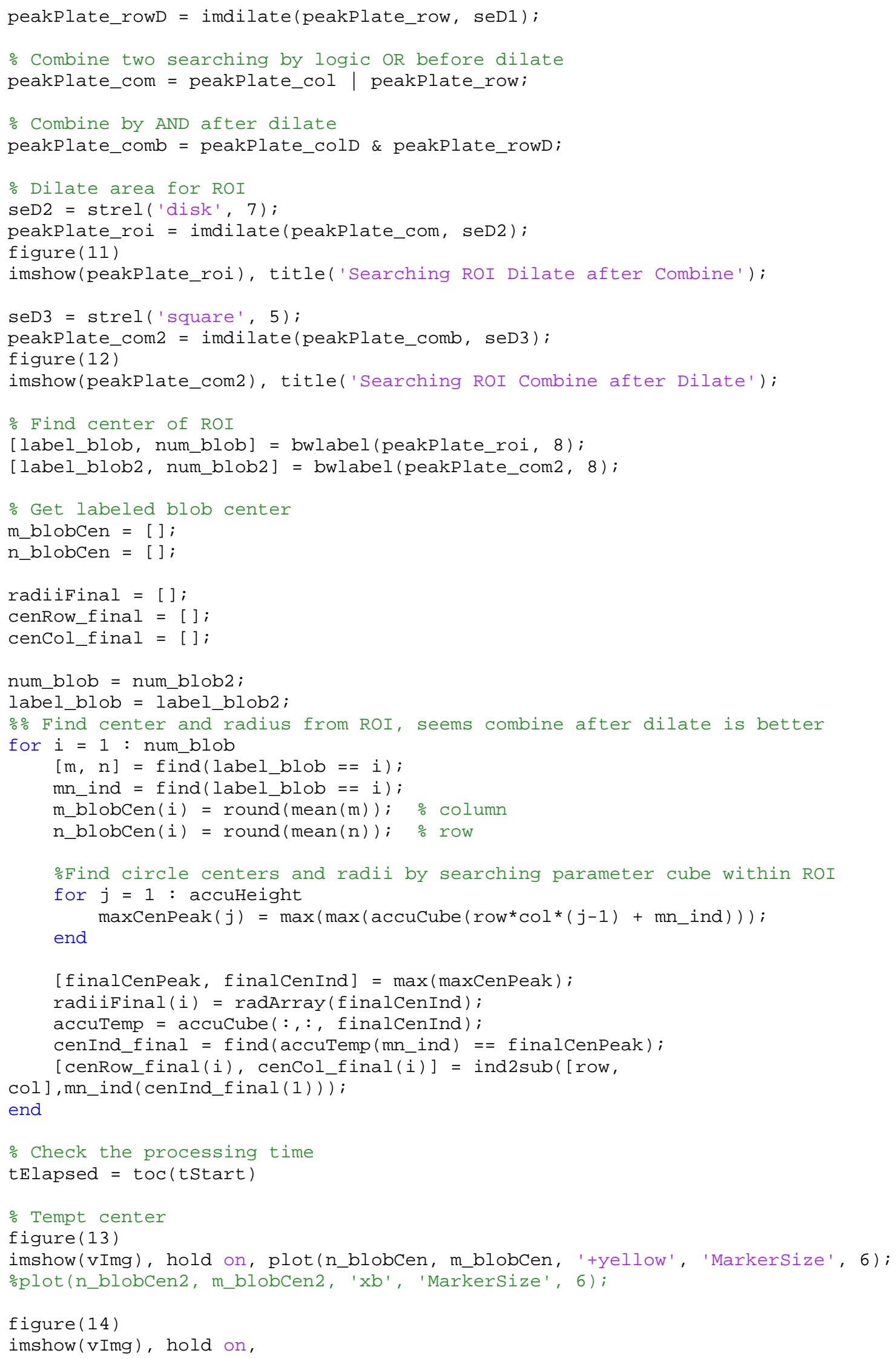


plot (cencol_final, cenRow_final, '+blue', 'Markersize', 6),

title(['Beads Detection by HT of ', img_title]);

for $i=1$ : num_blob

DrawCircle(cencol_final(i), cenRow_final(i), radiifinal(i), 360, 'blue'); end

text(cencol_final(i)+5, cenRow_final(i)+5, num2str(i), 'color', 'blue');

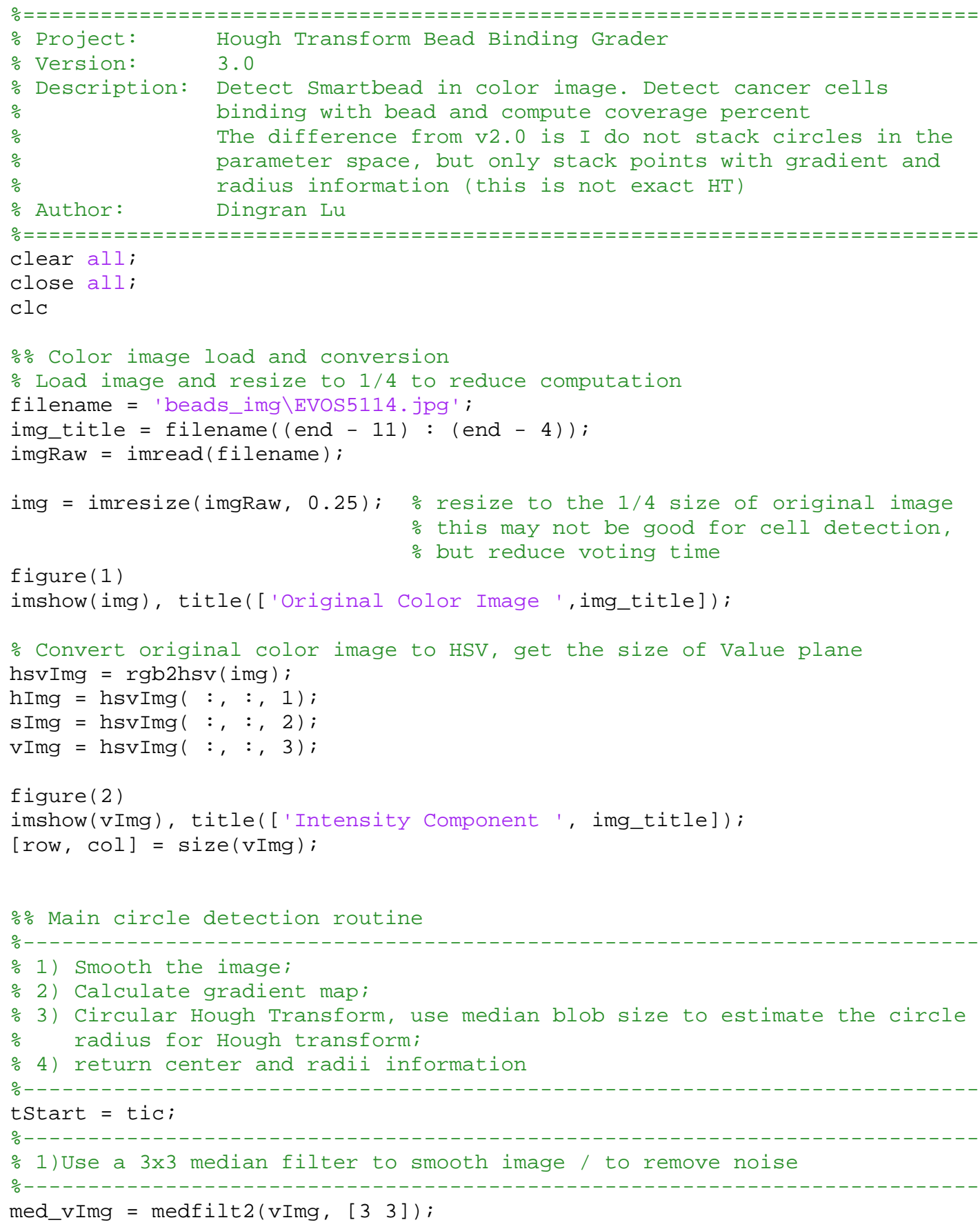




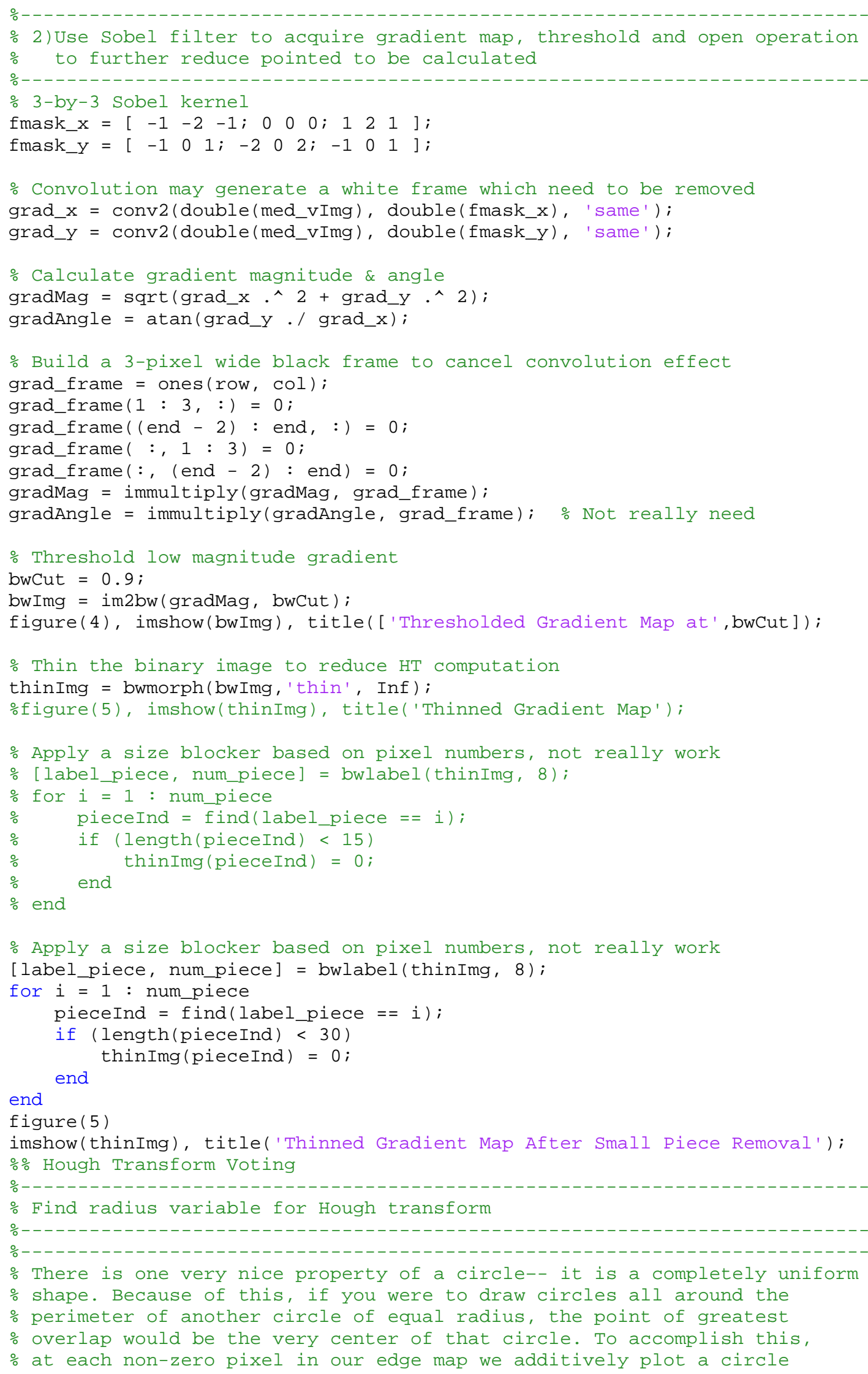




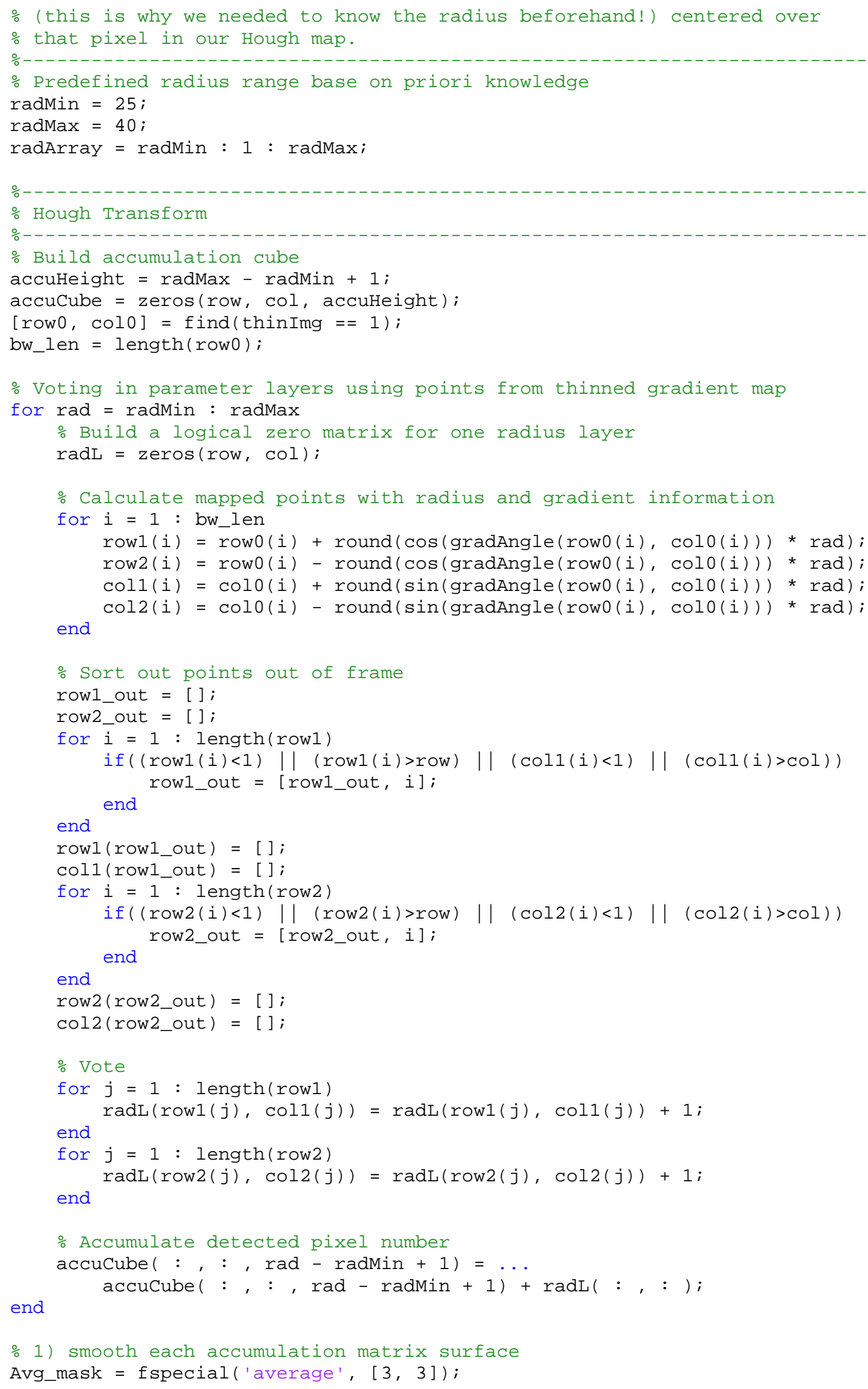




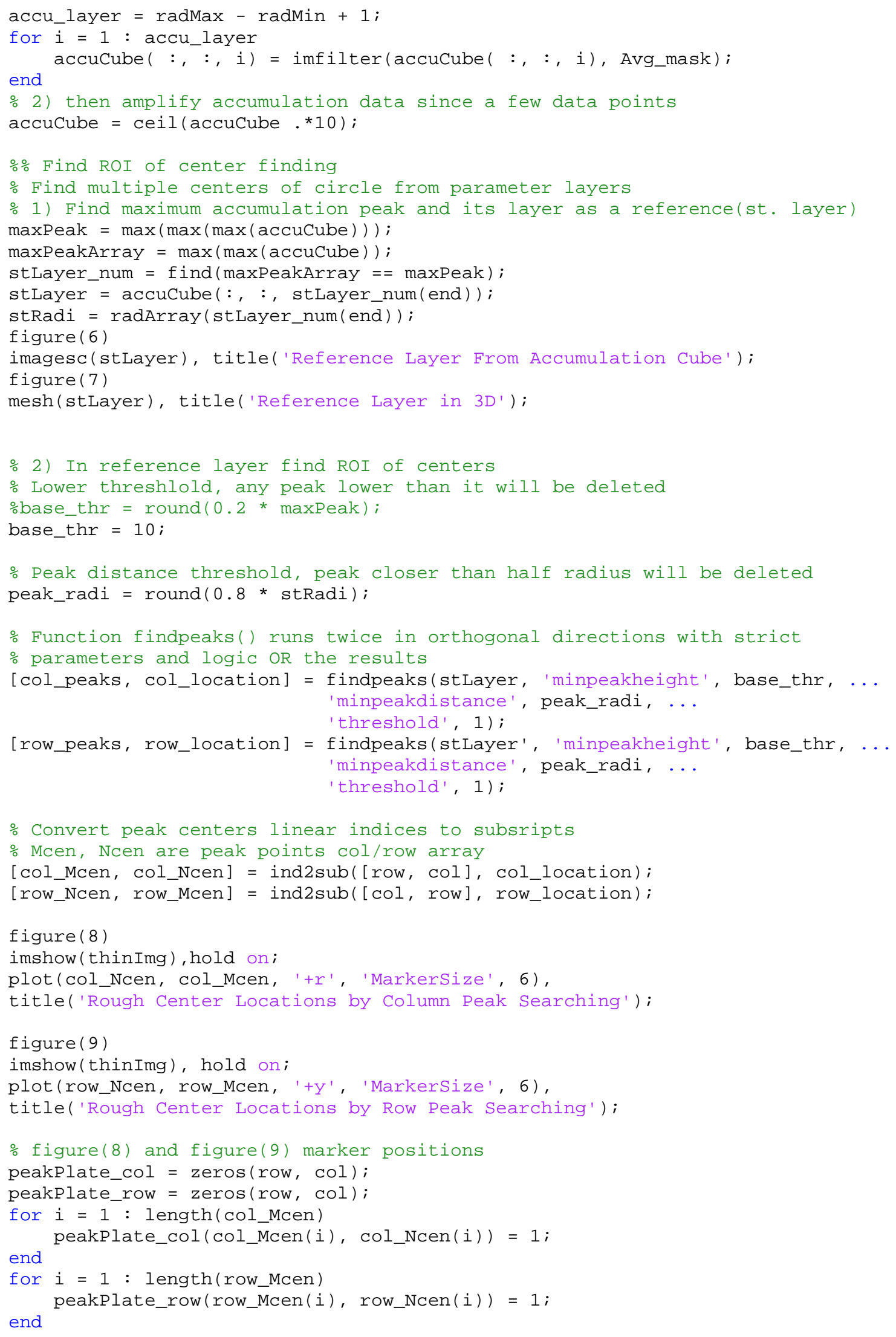




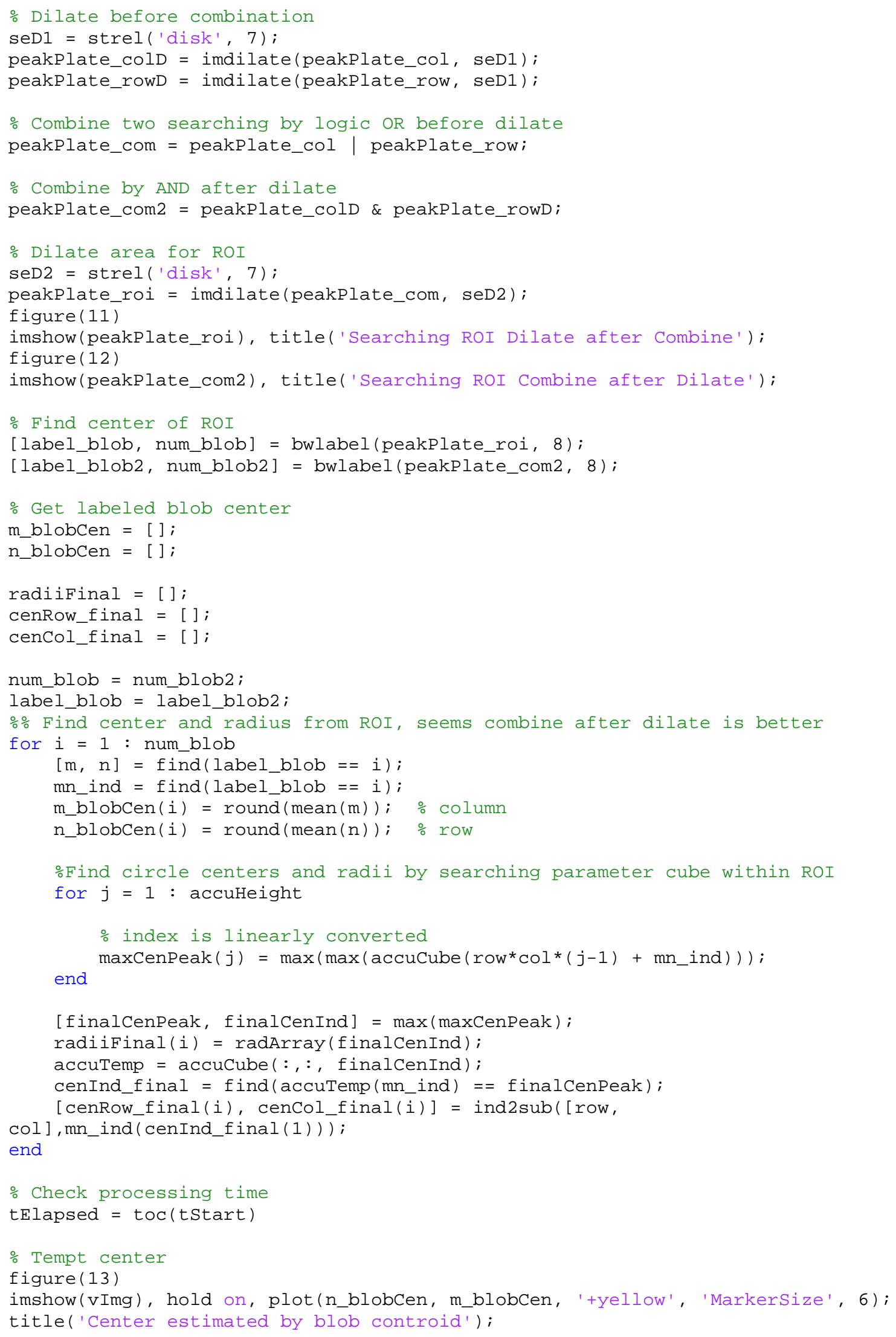


figure(14)

imshow(vImg), hold on,

plot (cenCol_final, cenRow_final, '+blue', 'Markersize', 6),

title(['Final Detected Beads ', img_title]);

for $i=1$ : num_blob

DrawCircle(cenCol_final(i), cenRow_final(i), radiifinal(i), 360, 'blue'); end text (cencol_final( $i)+5$, cenRow_final(i)+5, num2str(i), 'color', 'blue');

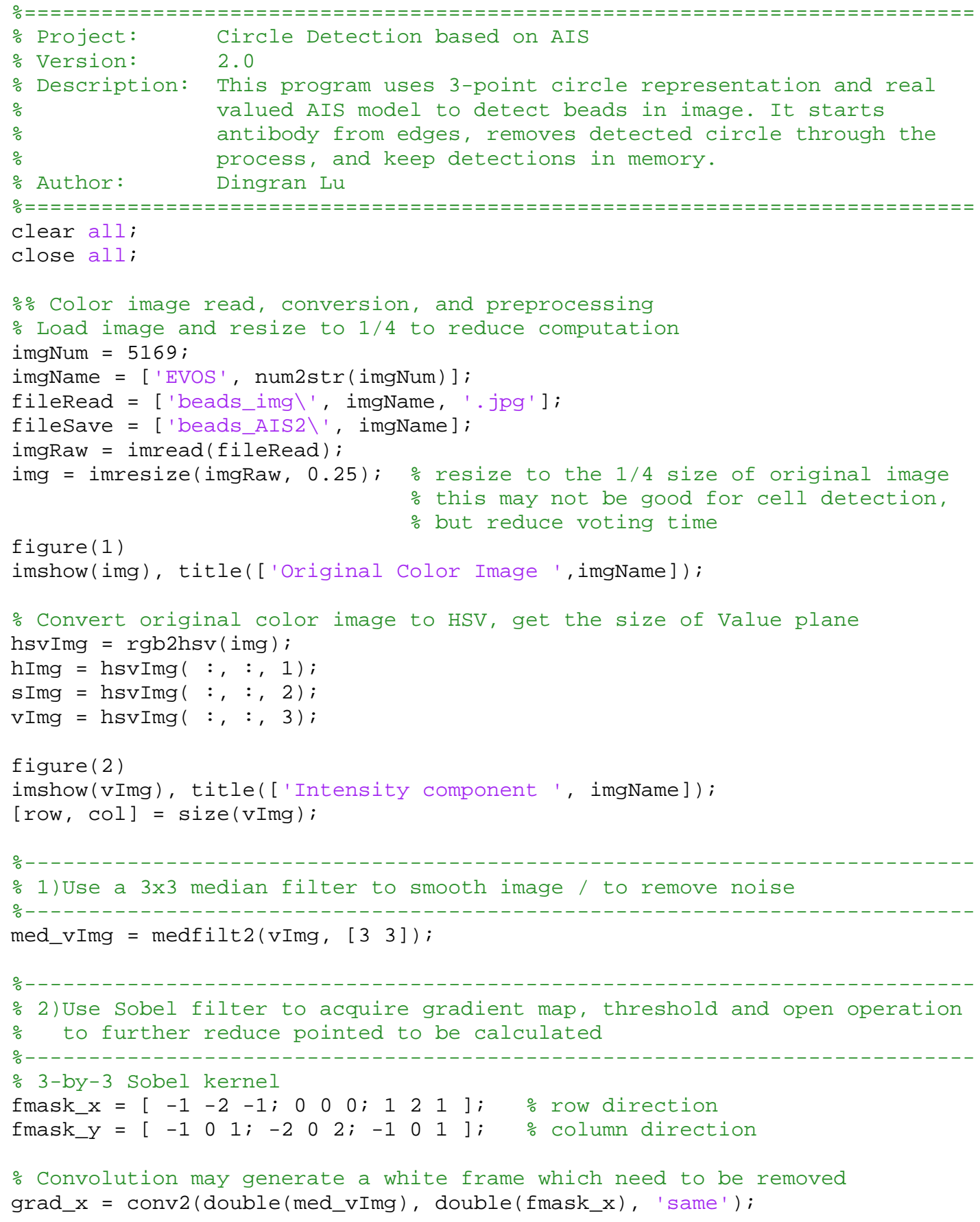




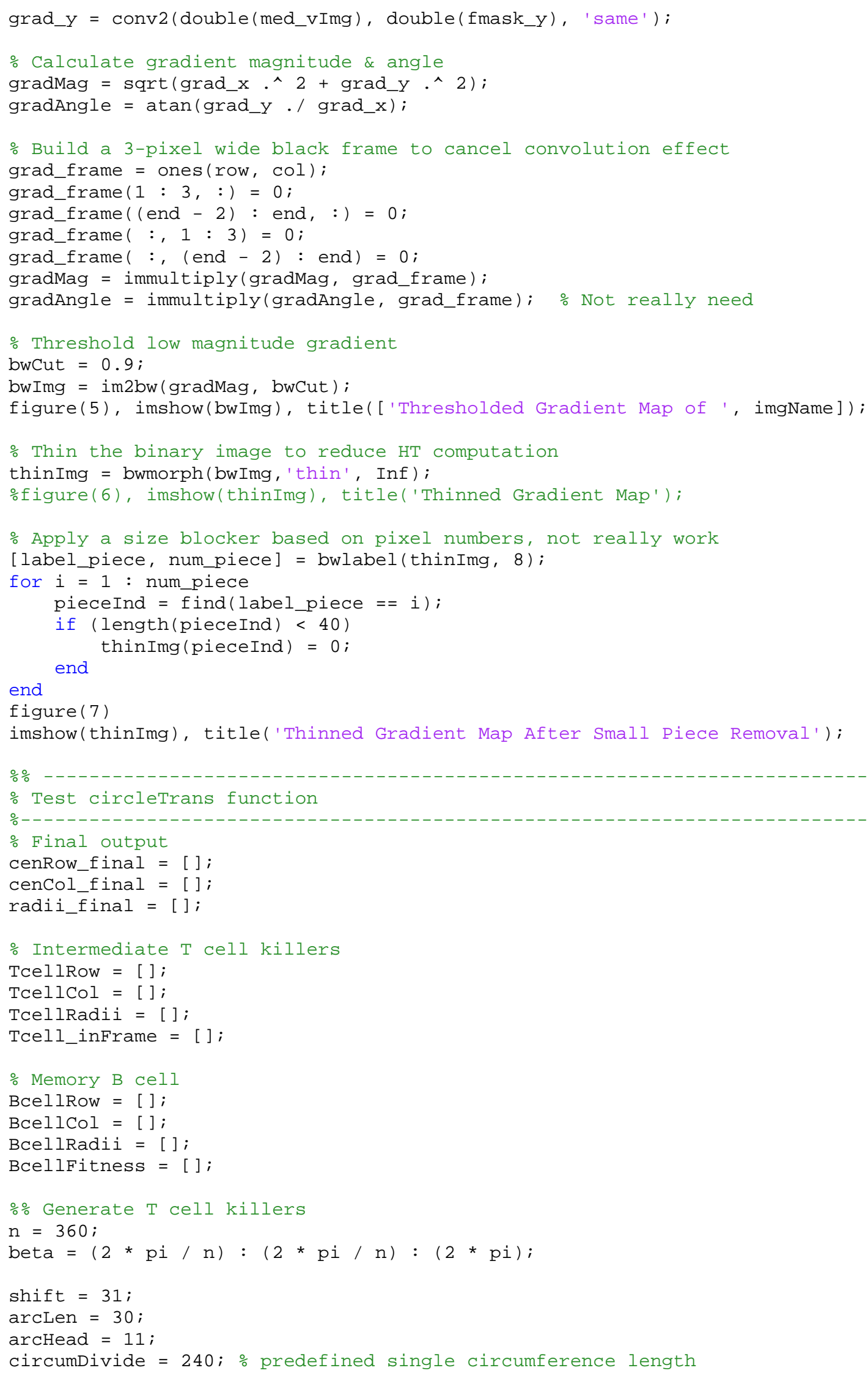




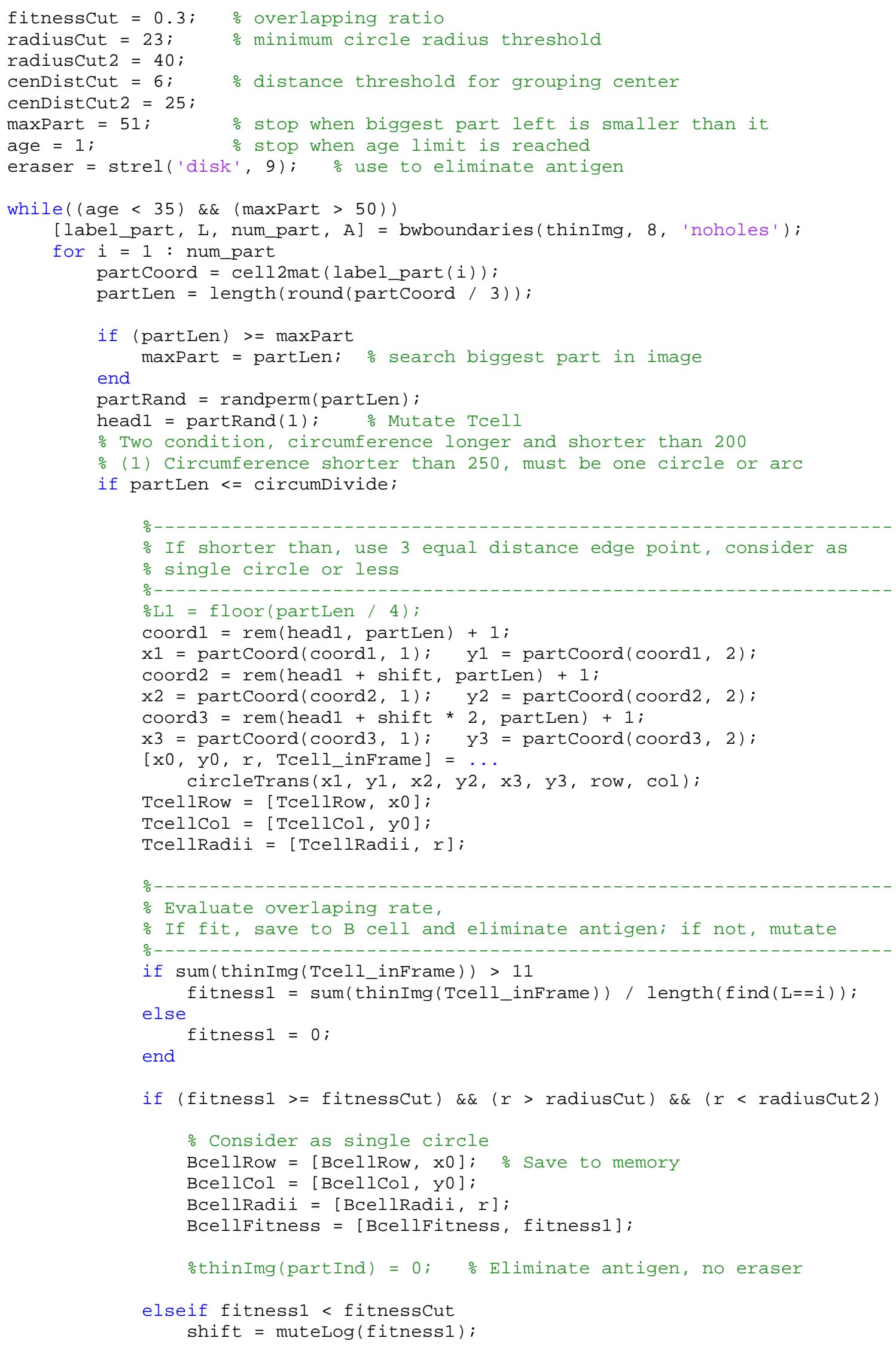


end

\% (2) Circumferen longer than 250, could be one circle, or multiple connected circles

\%-

$\%$ If longer than, consider as multiple circle

\%-_.

else

coord1 $=\operatorname{rem}($ head $1, \operatorname{partLen})+1 ;$

$\mathrm{x} 1=\operatorname{partcoord}(\operatorname{coord} 1,1) ; \quad \mathrm{y} 1=\operatorname{partCoord}(\operatorname{coord} 1,2) ;$

coord2 = rem(head1 + shift, partLen $)+1$;

$\mathrm{x} 2=\operatorname{partcoord}(\operatorname{coord} 2,1) ; \quad \mathrm{y} 2=\operatorname{partCoord}(\operatorname{coord} 2,2) ;$

coord3 $=$ rem $($ head1 + shift *2, partLen $)+1$;

$\mathrm{x} 3=\operatorname{partcoord}(\operatorname{coord} 3,1) ; \quad \mathrm{y} 3=\operatorname{partcoord}(\operatorname{coord} 3,2) ;$

$[x \odot, y \Theta, r$, Tcell_inframe $]=\ldots$

circleTrans(x1, y1, x2, y2, x3, y3, row, col);

TcellRow $=[$ TcellRow, $x \Theta]$;

TcellCol = $[$ TcellCol, yo $]$;

TcellRadii $=[$ TcellRadii, $r]$;

$\%$ Evaluate overlaping rate,

$\%$ If fit, save to B cell and eliminate antigen; if not, mutate

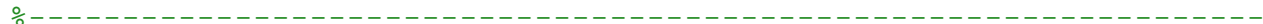

fitness2 $=\operatorname{sum}($ thinImg $($ Tcell_inFrame $)) /\left(2{ }^{*} i^{*} r\right) ; \%$ THIS IS

DIFFERENT

if $\operatorname{sum}($ thinImg(Tcell_inFrame)) $>11$

else

fitness1 = sum(thinImg $($ Tcell_inFrame $)) / \operatorname{length}($ find $(L==i))$;

end

fitness1 = 0 ;

if (fitness1 >= fitnesscut) \&\& ( $r>$ radiusCut) $\& \&$ ( $r<$ radiusCut2)

$\%$ Consider as single circle

BcellRow $=[$ BcellRow, $\mathrm{x} \odot] ; \%$ Save to memory

Bcellcol = [BcellCol, y०];

BcellRadii $=$ [BcellRadii, $r$ ];

BcellFitness $=$ [BcellFitness, fitness1];

\%thinImg $($ partInd $)=0 ; \quad \%$ Eliminate antigen

elseif (fitness1 $>0.1$ ) \&\& (fitness2 >= fitnessCut) $\ldots$ $\& \&$ ( $r>$ radiusCut $)$ \&\& $(r<$ radiusCut2)

\% Consider as a circle from multiple connected one

BcellRow $=[$ BcellRow, $x \odot] ; \%$ Save to memory

BcellCol = $[$ BcellCol, y०];

BcellRadii = [BcellRadii, r];

BcellFitness = [BcellFitness, fitness2];

elseif (fitness2 < fitnesscut)

end shift = mutelog(fitness1);

end

end

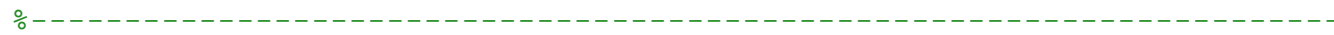

$\%$ Traverse whole memory, and group too close circle centers, rule is:

$\%$ if center distance closer than 5, keep the one with bigger radius

$\%$ if center distance bigger than 5, keep the one with higher fitness

\% 


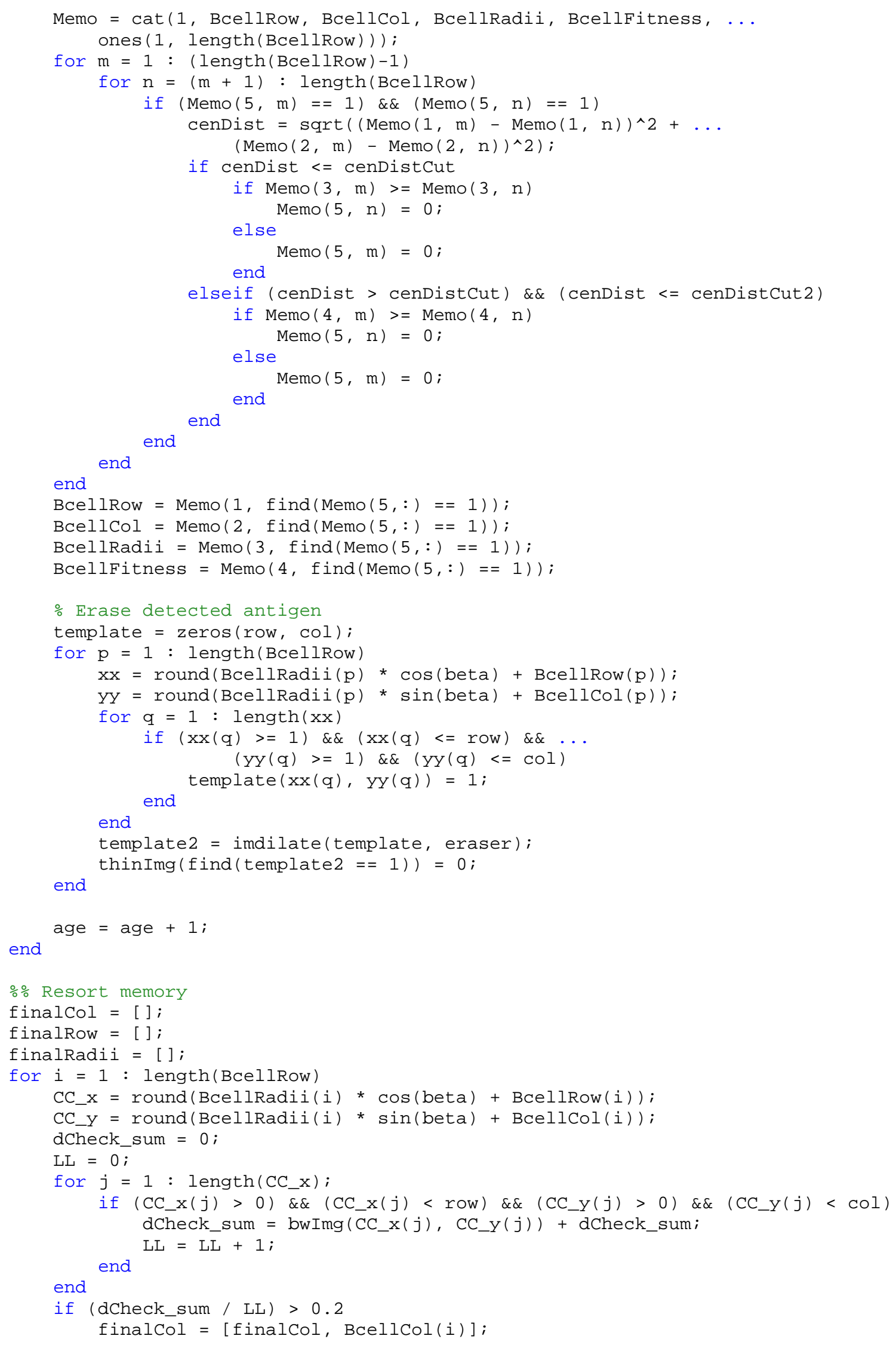




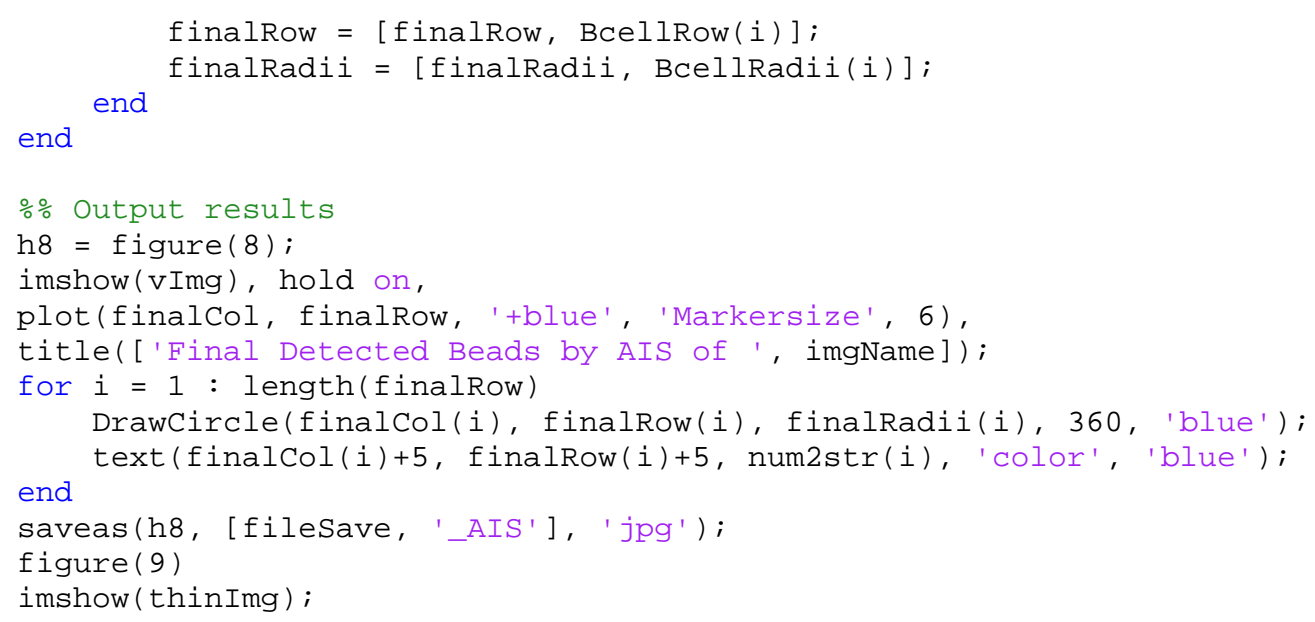

